\title{
THEORY OF REPRODUCING KERNELS $\left({ }^{(}\right)$
}

\author{
BY \\ N. ARONSZAJN
}

\section{PREFACE}

The present paper may be considered as a sequel to our previous paper in the Proceedings of the Cambridge Philosophical Society, Théorie générale de noyaux reproduisants - Première partie (vol. 39 (1944)) which was written in 1942-1943. In the introduction to this paper we outlined the plan of papers which were to follow. In the meantime, however, the general theory has been developed in many directions, and our original plans have had to be changed.

Due to wartime conditions we were not able, at the time of writing the first paper, to take into account all the earlier investigations which, although sometimes of quite a different character, were, nevertheless, related to our subject.

Our investigation is concerned with kernels of a special type which have been used under different names and in different ways in many domains of mathematical research. We shall therefore begin our present paper with a short historical introduction in which we shall attempt to indicate the different manners in which these kernels have been used by various investigators, and to clarify the terminology. We shall also discuss the more important trends of the application of these kernels without attempting, however, a complete bibliography of the subject matter.

In Part I, we shall discuss briefly the essential notions and results of our previous paper and give a further development of the theory in an abstract form. In Part II, we shall illustrate the results obtained in the first part by a series of examples which will give new developments of already known applications of the theory, as well as some new applications.

\section{TABLE OF CONTENTS}

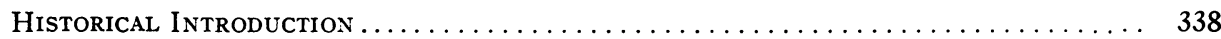

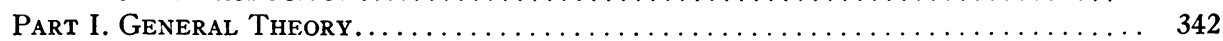

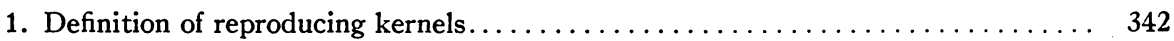

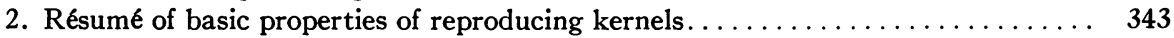

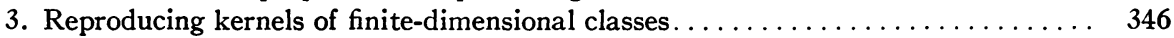

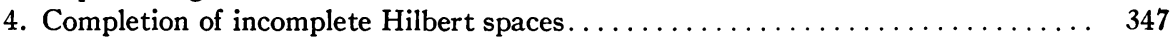

5. The restriction of a reproducing kernel $\ldots \ldots \ldots \ldots \ldots \ldots \ldots \ldots \ldots \ldots \ldots \ldots \ldots$

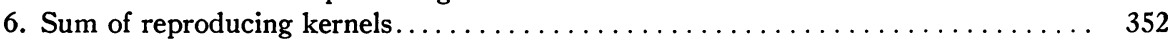

Presented to the Society, December 28, 1948, under the title Hilbert spaces and conformal mappings; received by the editors November 26, 1948.

(1) Paper done under contract with the Office of Naval Research, N5ori 76-16-NR 043-046, Division of Engineering Science, Harvard University. 


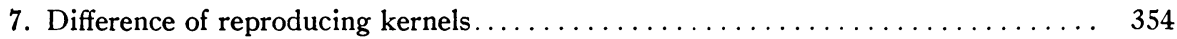

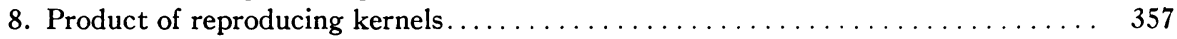

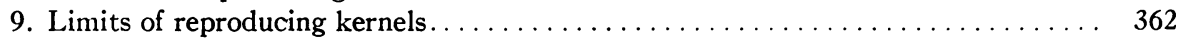

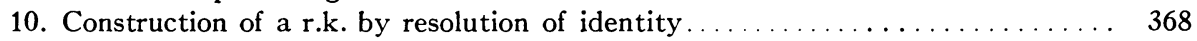

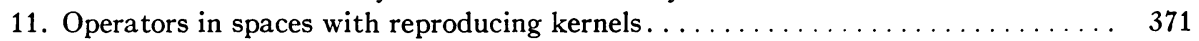

12. The reproducing kernel of a sum of two closed subspaces............. 375

13. Final remarks in the general theory ........................... 380

PART II. EXAMPLES... . . . . . . . . . . . . . . . . . . . . . . . . . . . . . . . . . . 384

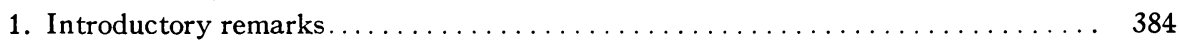

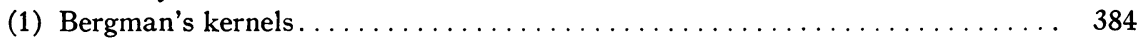

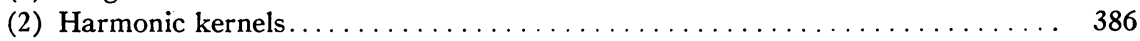

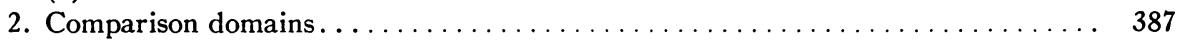

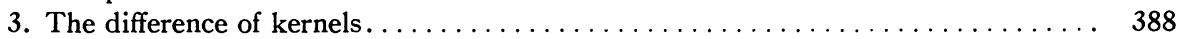

4. The square of a kernel introduced by Szegö. . . . . . . . . . . . . . . . . . . . 391

5. The kernel $H\left(z, z_{1}\right)$ for an ellipse . . . . . . . . . . . . . . . . . . . 393

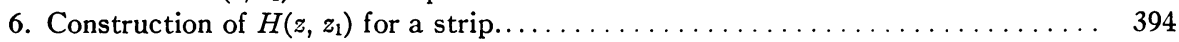

7. Limits of increasing sequences of kernels...................... 396

8. Construction of reproducing kernels by the projection-formula of $\$ 12, I \ldots \ldots \ldots 397$

BibLIOGRAPHY .............................................. 401

\section{Historical InTRODUCTION}

Examples of kernels of the type in which we are interested have been known for a long time, since all the Green's functions of self-adjoint ordinary differential equations (as also some Green's functions-the bounded onesof partial differential equations) belong to this type. But the characteristic properties of these kernels as we now understand them have only been stressed and applied since the beginning of the century.

There have been and continue to be two trends in the consideration of these kernels. To explain them we should mention that such a kernel $K(x, y)$ may be characterized as a function of two points, by a property discovered by J. Mercer [1] $\left(^{2}\right)$ in 1909 . To the kernel $K$ there corresponds a well determined class $F$ of functions $f(x)$, in respect to which $K$ possesses the "reproducing" property (E. H. Moore [2]). On the other hand, to a class of functions $F$, there may correspond a kernel $K$ with "reproducing" property (N. Aronszajn [4]).

Those following the first trend consider a given kernel $K$ and study it in itself, or eventually apply it in various domains (as integral equations, theory of groups, general metric geometry, and so on). The class $F$ corresponding to $K$ may be used as a tool of research, but is introduced a posteriori (as in the work of E. H. Moore [2], and more recently of A. Weil [1], I. Gelfand and D. Raikoff [1], and R. Godement [1, 2]). In the second trend, one is interested primarily in a class of functions $F$, and the corresponding kernel $K$ is used essentially as a tool in the study of the functions of this class. One of the basic problems in this kind of investigation is the explicit construction and computation of the kernel for a given class $F$.

(2) Numbers in brackets refer to the bibliography at the end of the paper. 
The first of these trends originated in the theory of integral equations as developed by Hilbert. The kernels considered then were continuous kernels of positive definite integral operators. This theory was developed by J. Mercer $[1,2]$ under the name of "positive definite kernels" and on occasion has been used by many others interested in integral equations, especially during the second decade of this century. Mercer discovered the property

$$
\sum_{i, j=1}^{n} K\left(y_{i}, y_{j}\right) \bar{\xi}_{i} \xi_{j} \geqq 0, \quad y_{i} \text { any points, } \xi_{i} \text { any complex numbers }\left(^{3}\right),
$$

characterizing his kernels, among all the continuous kernels of integral equations. To this same trend belong the investigations of E. H. Moore [1, 2] who, during the second and third decades of the century, introduced these kernels in the general analysis under the name of "positive hermitian matrices" with a view to applications in a kind of generalization of integral equations. Moore considered kernels $K(x, y)$ defined on an abstract set $E$ and characterized by the property (1). He discovered the theorem now serving as one of the links between the two trends, proving that to each positive hermitian matrix there corresponds a class of functions forming what we now call a Hilbert space with a scalar product $(f, g)$ and in which the kernel has the reproducing property.

$$
f(y)=(f(x), K(x, y)) .
$$

Also to the same trend (though seemingly without any connection to previous investigations) belongs the notion introduced by S. Bochner [2] during the third decade of the century under the name of "positive definite functions." Bochner considered continuous functions $\phi(x)$ of real variable $x$ such that the kernels $K(x, y)=\phi(x-y)$ conformed to condition (1). He introduced these functions with a view to application in the theory of Fourier transforms. The notion was later generalized by A. Weil [1] and applied by I. Gelfand and D. Raikoff [1], R. Godement [1, 2], and others to the investigation of topological groups under the name of positive definite functions or functions of positive type. These functions were also applied to general metric geometry (the Hilbert distances) by I. J. Schoenberg [1, 2], J. v. Neumann and I. J. Schoenberg [1], and S. Bochner [3].

The second trend was initiated during the first decade of the century in the work of S. Zaremba [1, 2] on boundary value problems for harmonic and biharmonic functions. Zaremba was the first to introduce, in a particular case, the kernel corresponding to a class of functions, and to state its reproducing property (2). However, he did not develop any general theory, nor did he give any particular name to the kernels he introduced. It appears that nothing was done in this direction until the third decade when S. Berg-

(3) Mercer used only real numbers $\xi_{i}$ as he considered only real kernels $K$. 
man [1] introduced kernels corresponding to classes of harmonic functions and analytic functions in one or several variables. He called these "kernel functions." They were introduced as kernels of orthogonal systems in these classes for an adequate metric. The reproducing property of these kernels was noticed by Bergman [1] (also by N. Aronszajn [1]), but it was not used as their basic characteristic property as is done at present.

In the third and fourth decades most of the work was done with kernels which we shall call Bergman's kernels, that is, kernels of classes of analytic functions $f$ of one or several complex variables, regular in a domain $D$ with the quadratic metric

$$
\int_{D}|f|^{2} d \tau
$$

A quantity of important results were achieved by the use of these kernels in the theory of functions of one and several complex variables (Bergman $[4,6,7]$, Bochner [1]), in conformal mapping of simply- and multiply-connected domains (Bergman [11, 12], Zarankiewicz [1] and others), in pseudoconformal mappings (Bergman [4, 5, 8, 9], Welke [1], Aronszajn [1], and others), in the study of invariant Riemannian metrics (Bergman [11, 14], Fuchs $[1,2])$, and in other subjects.

The original idea of Zaremba to apply the kernels to the solution of boundary value problems was represented in these two decades by only a few papers of Bergman $[1,2,3,10]$. Only since the last war has this idea been put into the foreground by a series of papers by Bergman [13], and Bergman and Schiffer $[1,2,3]$. In these investigations, the kernel was proved to be a powerful tool for solving boundary value problems of partial differential equations of elliptic type. By the use of variational methods going back to Hadamard, relations were established between the kernels corresponding to classes of solutions of different equations and for different domains (Bergman and Schiffer $[1,3])$. For a partial differential equation, the kernel of the class of solutions in a domain was proved to be the difference of the corresponding Neumann's and Green's functions (Bergman and Schiffer $[1,3]$ ) (in the special case of the biharmonic equation a relation of this kind was already noticed by Zaremba). Parallel to this revival of the application of kernels to partial differential equations there is developing a study of the relationship between these kernels and Bergman's kernels of analytic functions (Bergman [12], M. Schiffer [1, 2]). Also the application of kernels to conformal mapping of multiply-connected domains has made great progress as all the important mapping functions were proved to be simply expressible by the Bergman's kernel (Bergman [11, 12], P. Garabedian and M. Schiffer [1], Garabedian $[1]$, and $Z$. Nehari $[1,2])$. Quite recently, the connection was found between the Bergman's kernel and the kernel introduced by G. Szegö (P. Garabedian [1]).

In 1943, the author ([4] also [6]) developed the general theory of repro- 
ducing kernels which contains, as particular cases, the Bergman kernelfunctions. This theory gives a general basis for the study of each particular case and allows great simplification of many of the proofs involved. In this theory a central role is played by the reproducing property of the kernel in respect to the class to which it belongs. The kernel is defined by this property. The simple fact was stressed that a reproducing kernel always possesses property (1) characteristic of positive hermitian matrices (in the sense of E. H. Moore). This forms the second link between the two trends in the kernel theory (the theorem of E. H. Moore forming the first link was mentioned above).

The mathematicians working in the two trends seem not to have noticed the essential connections between the general notions they were using. At present the two concepts of the kernel, as a positive hermitian matrix and as a reproducing kernel, are known to be equivalent and methods elaborated in the investigations belonging to one trend prove to be of importance in the other.

We should like to elaborate here briefly on the matter of the terminology which has been used by various investigators. As we have seen above, different names have been given to the kernels in which we are interested. When the kernels were used in themselves, without special or previous consideration of the class to which they belonged, they were called "positive definite kernels," "positive hermitian matrices," "positive definite functions," or "functions of positive type." In cases where they were considered as determined, and in connection with a class of functions, they were called "kernel functions" or "reproducing kernels." It is not our intention to settle here the question of terminology. Our purpose is rather to state our choice and to give our reasons for it together with a comparison of the terminology we have chosen with that used by other authors.

It would seem advisable to keep two names for our kernels, the function of each name being to indicate immediately in what context the kernel under consideration is to be taken. Thus, when we consider the kernel in itself we shall call it (after E. H. Moore) a positive matrix $\left({ }^{4}\right)$, in abbreviation, p. matrix, or $p . m$. When we wish to indicate the kernel corresponding to a class of functions we shall call it the reproducing kernel of the class, in abbreviation, $r$. kernel or $r . k$.

As compared to other terminology, we believe that the name "positive definite function" or perhaps better "function of positive type" will probably continue to be used in the particular case when the kernel is of the form $\phi(x-y), x, y$ belonging to an additive group. This term has been used in a few instances for some more general kernels, but we believe that it would prove to be more convenient if it were restricted to the particular case

(4) We drop here the adjective "hermitian" since the condition that the quadratic form (1) be positive implies the hermitian symmetry of the matrix. 
mentioned above.

Although the name of "positive definite kernels" would seem, somehow, more adequate than "positive matrices," especially since it was introduced first, we have chosen rather the term used by E. H. Moore. This is because we wish to reserve the notion of positive definite kernel for more general kernels which would include the positive definite matrices as well as some other non-bounded kernels (such as the kernels of general positive definite integral operators and also the recently introduced pseudo-reproducing kernels [Aronszajn 5, 6]).

On the other hand, when we have in mind the kernel corresponding to a given class of functions, the simplest terminology is to call it "the kernel of the class" or "the kernel belonging to the class." But when some ambiguity is to be feared, or when we wish to stress its characteristic property, we use the adjective "reproducing."

\section{Part I. General theory}

1. Definition of reproducing kernels. Consider a linear class $F$ of functions $f(x)$ defined in a set $E$. We shall suppose that $F$ is a complex class, that is, that it admits of multiplication by complex constants.

Suppose further that for $f \in F$ is defined a norm $\|f\|$ (that is, a real number satisfying: $\|f\| \geqq 0,\|f\|=0$ only for $f=0,\|c f\|=|c|\|f\|,\|f+g\| \leqq\|f\|+\|g\|)$ given by a quadratic hermitian form $Q(f)$

$$
\|f\|^{2}=Q(f) .
$$

Here, a functional $Q(f)$ is called quadratic hermitian if for any constants $\xi_{1}, \xi_{2}$ and functions $f_{1}, f_{2}$ of $F$

$$
Q\left(\xi_{1} f_{1}+\xi_{2} f_{2}\right)=\left|\xi_{1}\right|^{2} Q\left(f_{1}\right)+\xi_{1} \bar{\xi}_{2} Q\left(f_{1}, f_{2}\right)+\bar{\xi}_{1} \xi_{2} Q\left(f_{2}, f_{1}\right)+\left|\xi_{2}\right|^{2} Q\left(f_{2}\right) .
$$

$Q\left(f_{1}, f_{2}\right)=\overline{Q\left(f_{2}, f_{1}\right)}$ is the uniquely determined bilinear hermitian form corresponding to the quadratic form $Q(f)$. This bilinear form will be denoted by $\left(f_{1}, f_{2}\right) \equiv Q\left(f_{1}, f_{2}\right)$ and called the scalar product corresponding to the norm $\|f\|$ (or the quadratic metric $\|f\|^{2}$ ). We have

$$
\|f\|^{2}=(f, f) \text {. }
$$

The class $F$ with the norm, \|\| , forms a normed complex vector space. If this space is complete it is a Hilbert space.

If $F$ is a class of real-valued functions forming a real vector space (that is, admitting of multiplication with only real constants), if the norm, \|\| , in $F$ is given by $\|f\|^{2}=Q(f)$ with an ordinary quadratic form $Q$ (that is, for real $\xi_{1}, \xi_{2}, Q\left(\xi_{1} f_{1}+\xi_{2} f_{2}\right)=\xi_{1}^{2} Q\left(f_{1}\right)+2 \xi_{1} \xi_{2} Q\left(f_{1}, f_{2}\right)+\xi_{2}^{2} Q\left(f_{2}\right)$, where $Q\left(f_{1}, f_{2}\right)$ is the corresponding bilinear symmetric form), and if $F$ is a complete space, it is a real Hilbert space. The scalar product is given there by $\left(f_{1}, f_{2}\right) \equiv Q\left(f_{1}, f_{2}\right)$.

Every class $F$ of real functions forming a real Hilbert space determines a 
complex Hilbert space in the following way: consider all functions $f_{1}+i f_{2}$ with $f_{1}$ and $f_{2}$ in $F$. They form a complex vector space $F_{c}$ in which we define the norm by $\left\|f_{1}+i f_{2}\right\|^{2}=\left\|f_{1}\right\|^{2}+\left\|f_{2}\right\|^{2}$. $F_{c}$ is a complex Hilbert space.

The complex Hilbert spaces $F$ determined in this way by real Hilbert spaces are characterized by the two properties:

(1) if $f \in F, \bar{f} \in F(\bar{f}$ is the conjugate complex function of $f$ ),

(2) $\|f\|=\bar{f}$.

Let $F$ be a class of functions defined in $E$, forming a Hilbert space (complex or reai). The function $K(x, y)$ of $x$ and $y$ in $E$ is called a reproducing kernel $(r . k$.) of $F$ if

1. For every $y, K(x, y)$ as function of $x$ belongs to $F$.

2. The reproducing property: for every $y \in E$ and every $f \in F$,

$$
f(y)=(f(x), K(x, y))_{x} .
$$

The subscript $x$ by the scalar product indicates that the scalar product applies to functions of $x$.

If a real class $F$ possesses a r.k. $K(x, y)$ then it is immediately verified that the corresponding complex space $F_{c}$ possesses the same kernel (which is realvalued):

From now on (unless otherwise stated) we shall consider only complex Hilbert spaces. As we have seen there is no essential limitation in this assumption.

It will be useful to introduce a distinction between the terms subclass and subspace. When $F_{1}$ and $F_{2}$ are two classes of functions defined in the same set $E, F_{1}$ is a subclass of $F_{2}$ if every $f$ of $F_{1}$ belongs to $F_{2}$. $F_{1}$ is a subspace of $F_{2}$ if it is a subclass of $F_{2}$ and if for every $f \in F_{1},\|f\|_{1}=\|f\|_{2}\left(\|\quad\|_{1}\right.$ and \|\|$_{2}$ are the norms in $F_{1}$ and $F_{2}$ respectively). $F_{1} \subset F_{2}$ means that $F_{1}$ is a subclass of $F_{2}$.

2. Résumé of basic properties of reproducing kernels. In the following, $F$ denotes a class of functions $f(x)$ defined in $E$, forming a Hilbert space with the norm $\|f\|$ and scalar product $\left(f_{1}, f_{2}\right) . K(x, y)$ will denote the corresponding reproducing kernel.

The detailed proofs of the properties listed below may be found in [Aronszajn, 4].

(1) If a r.k. $K$ exists it is unique. In fact, if another $K^{\prime}(x, y)$ existed we would have for some $y$

$$
\begin{aligned}
0<\left\|K(x, y)-K^{\prime}(x, y)\right\|^{2} & =\left(K-K^{\prime}, K-K^{\prime}\right) \\
& =\left(K-K^{\prime}, K\right)-\left(K-K^{\prime}, K^{\prime}\right)=0
\end{aligned}
$$

because of the reproducing property of $K$ and $K^{\prime}$.

(2) Existence. For the existence of a r.k. $K(x, y)$ it is necessary and sufficient that for every $y$ of the set $E, f(y)$ be a continuous functional of $f$ running through the Hilbert space $F$.

In fact, if $K$ exists, then $|f(y)| \leqq\|f\|(K(x, y), K(x, y))^{1 / 2}=K(y, y)^{1 / 2}\|f\|$. 
On the other hand if $f(y)$ is a continuous functional, then by the general theory of the Hilbert space there exists a function $g_{y}(x)$ belonging to $F$ such that $f(y)=\left(f(x), g_{y}(x)\right)$, and then if we put $K(x, y)=g_{y}(x)$ it will be a reproducing kernel.

(3) $K(x, y)$ is a positive matrix in the sense of $\mathrm{E}$. H. Moore, that is, the quadratic form in $\xi_{1}, \cdots, \xi_{n}$

$$
\sum_{i, j=1}^{n} K\left(y_{i}, y_{j}\right) \bar{\xi}_{i} \xi_{j}
$$

is non-negative for all $y_{1}, \cdots, y_{n}$ in $E$. This is clear since expression (1) equals $\left\|\sum_{1}^{n} K\left(x, y_{i}\right) \xi_{i}\right\|^{2}$, following the reproducing property. In particular it follows that

$$
K(x, x) \geqq 0, \quad K(x, y)=\overline{K(y, x)}, \quad|K(x, y)|^{2} \leqq K(x, x) K(y, y) .
$$

(4) The theorem in (3) admits a converse due essentially to E. H. Moore: to every positive matrix $K(x, y)$ there corresponds one and only one class of functions with a uniquely determined quadratic form in it, forming a Hilbert space and admitting $K(x, y)$ as a reproducing kernel.

This class of functions is generated by all the functions of the form $\sum \alpha_{k} K(x, y)$. The norm of this function is defined by the quadratic form $\left\|\sum \alpha_{k} K\left(x, y_{k}\right)\right\|^{2}=\sum \sum K\left(y_{i}, y_{j}\right) \bar{\xi}_{i} \xi_{j}$. Functions with this norm do not as yet form a complete Hilbert space, but it can be easily seen that they may be completed by the adjunction of functions to form such a complete Hilbert space. This follows from the fact that every Cauchy sequence of these functions (relative to the above norm) will converge at every point $x$ towards a limit function whose adjunction to the class will complete the space.

(5) If the class $F$ possesses a r.k. $K(x, y)$, every sequence of functions $\left\{f_{n}\right\}$ which converges strongly to a function $f$ in the Hilbert space $F$, converges also at every point in the ordinary sense, $\lim f_{n}(x)=f(x)$, this convergence being uniform in every subset of $E$ in which $K(x, x)$ is uniformly bounded. This follows from

$$
\begin{aligned}
\left|f(y)-f_{n}(y)\right| & =\left|\left(f(x)-f_{n}(x), K(x, y)\right)\right| \leqq\left\|f-f_{n}\right\|\|K(x, y)\| \\
& =\left\|f-f_{n}\right\|_{1}(K(y, y))^{1 / 2} .
\end{aligned}
$$

If $f_{n}$ converges weakly to $f$, we have again $f_{n}(y) \rightarrow f(y)$ for every $y$ (since, by the definition of the weak convergence, $\left(f_{n}(x), K(x, y)\right) \rightarrow(f(x), K(x, y))$. There is in general no increasing sequence of sets $E_{1} \subset E_{2} \subset \cdots \rightarrow E$ in each of which $f_{n}$ converges uniformly to $f$.

If a topology (a notion of limit) is defined in $E$ and if the correspondence $y \leftrightarrow K(x, y)$ transforms $E$ in a continuous manner into a subset of the space $F$, then the weakly convergent sequence $\left\{f_{n}\right\}$ converges uniformly in every compact set $E_{1} \subset E$. In fact $E_{1}$ is transformed into a compact subset of the 
space $F$. For every $\epsilon>0$ we can then choose a finite set $\left(y_{1}, \cdots, y_{l}\right) \subset E_{1}$ so that for every $y \in E_{1}$ there exists at least one $y_{k}$ with $\left\|K(x, y)-K\left(x, y_{k}\right)\right\|$ $\leqq \epsilon / 4 M$, where $M=$ l.u.b. $\left\|f_{n}\right\|$.

Further, if we choose $n_{0}$ so that for $n>n_{0},\left|f\left(y_{k}\right)-f_{n}\left(y_{k}\right)\right|<\epsilon / 4$, we shall obtain for $y \in E_{1}$.

$$
\begin{aligned}
\left|f(y)-f_{n}(y)\right| & =\left|\left(f(y)-f\left(y_{k}\right)\right)+\left(f\left(y_{k}\right)-f_{n}\left(y_{k}\right)\right)+\left(f_{n}\left(y_{k}\right)-f_{n}(y)\right)\right| \\
& \leqq\left|f\left(y_{k}\right)-f_{n}\left(y_{k}\right)\right|+\left|\left(f(x)-f_{n}(x), K(x, y)-K\left(x, y_{k}\right)\right)\right| \\
& \leqq \frac{\epsilon}{4}+\left\|f-f_{n}\right\| \| K(x, y)-K\left(x, y_{k}\right)|| \frac{\epsilon}{4}+2 M \frac{\epsilon}{4 M}<\epsilon .
\end{aligned}
$$

The continuity of the correspondence $y \leftrightarrow K(x, y)$ is equivalent to equicontinuity of all functions of $F$ with $\|f\|<M$ for any $M>0$. This property is satisfied by most of the classes with reproducing kernels which are usually considered (such as classes of analytic functions, harmonic functions, solutions of partial differential equations, and so on).

(6) If the class $F$ with the r.k. $K$ is a subspace of a larger Hilbert space $\mathfrak{S}$, then the formula

$$
f(y)=(h, K(x, y))_{x}
$$

gives the projection $f$ of the element $h$ of $\mathfrak{S}$ on $F$.

In fact $h=f+g$, where $g$ is orthogonal to the class $F . K(x, y)$ as a function of $x$ belongs to $F$ and so we have $(h, K(x, y))=(f+g, K(x, y))=(f, K(x, y))$ $=f(y)$ by the reproducing property.

(7) If $F$ possesses a r.k. $K$, then the same is true of all closed linear subspaces of $F$, because if $f(y)$ is a continuous functional of $f$ running through $F$, it is so much the more so if $f$ runs through a subclass of $F$. If $F^{\prime}$ and $F^{\prime \prime}$ are complementary subspaces of $F$, then their reproducing kernels satisfy the equation $K^{\prime}+K^{\prime \prime}=K$.

(8) If $F$ possesses a r.k. $K$ and if $\left\{g_{n}\right\}$ is an orthonormal system in $F$, then for every sequence $\left\{\alpha_{n}\right\}$ of numbers satisfying

$$
\sum_{1}^{\infty}\left|\alpha_{n}\right|^{2}<\infty
$$

we have

$$
\sum_{1}^{\infty}\left|\alpha_{n}\right|\left|g_{n}(x)\right| \leqq K(x, x)^{1 / 2}\left(\sum_{1}^{\infty}\left|\alpha_{n}\right|^{2}\right)^{1 / 2} .
$$

In fact, for a fixed $y$, the Fourier coefficients of $K(x, y)$ for the system $\left\{g_{n}\right\}$ are

$$
\left(K(x, y), g_{n}(x)\right)=\overline{\left(g_{n}(x), K(x, y)\right)}=\overline{g_{n}(y)} .
$$


Consequently

$$
\sum_{1}^{\infty} g_{n}(y)^{2} \leqq(K(x, y), K(x, y))_{x}=K(y, y) .
$$

Therefore

$$
\begin{aligned}
\sum_{1}^{\infty}\left|\alpha_{n}\right|\left|g_{n}(x)\right| & \leqq\left(\sum_{1}^{\infty}\left|\alpha_{n}\right|^{2}\right)^{1 / 2}\left(\sum_{1}^{\infty}\left|g_{n}(x)\right|^{2}\right)^{1 / 2} \\
& \leqq K(x, x)^{1 / 2}\left(\sum_{1}^{\infty}\left|\alpha_{n}\right|^{2}\right)^{1 / 2}
\end{aligned}
$$

3. Reproducing kernels of finite-dimensional classes. If $F$ is of finite dimension $n$, let $w_{1}(x), \cdots, w_{n}(x)$ be $n$ linearly independent functions of $F$. All functions $f(x)$ of $F$ are representable in a unique manner as

$$
f(x)=\sum_{1} \zeta_{k} w_{k}(x), \quad \zeta_{k} \text { complex constants. }
$$

The most general quadratic metric in $F$ will be given by a positive definite hermitian form

$$
\|f\|^{2}=\sum_{i, j=1}^{n} \alpha_{i j} \zeta_{i} \bar{\zeta}_{j} .
$$

The scalar product has the form

$$
(f, g)=\sum_{i, j} \alpha_{i j} \zeta_{i} \bar{\eta}_{j}, \quad \quad \text { where } g=\sum \eta_{k} w_{k} .
$$

It is clear that

$$
\alpha_{i j}=\left(w_{i}, w_{j}\right) .
$$

Therefore the matrix $\left\{\alpha_{i j}\right\}$ is the Gramm's matrix of the system $\left\{w_{k}\right\}$. This matrix always possesses an inverse. Denote by $\left\{\beta_{i j}\right\}$ the conjugate of this inverse matrix. We have then

$$
\sum_{i} \alpha_{i} \vec{\beta}_{j k}=0 \text { or } 1 \text { following as } i \neq k \text { or } i=k .
$$

It is immediately verified that the function

$$
K(x, y)=\sum_{i, j=1}^{n} \beta_{i j} w_{i}(x) \overline{w_{j}(y)}
$$

is the reproducing kernel of the class $F$ with the metric (2).

The matrix $\left\{\beta_{i j}\right\}$ is hermitian positive definite. From the preceding developments we get, clearly, the following theorem. 
Theorem. $A$ function $K(x, y)$ is the reproducing kernel of a finite-dimensional class of functions if and only if it is of the form (6) with a positive definite matrix $\left\{\beta_{i j}\right\}$ and linearly independent functions $w_{k}(x)$. The corresponding class $F$ is then generated by the functions $w_{k}(x)$, the functions $f \in F$ given by (1) and the corresponding norm $\|f\|$ given by (2), where $\left\{\alpha_{i j}\right\}$ is the inverse matrix of $\left\{\overline{\beta_{i j}}\right\}$.

4. Completion of incomplete Hilbert spaces. In applications, we of ten meet classes of functions forming incomplete Hilbert spaces, that is, linear classes, with a scalar product, satisfying all the conditions for a Hilbert space with the exception of the completeness. For such classes, two problems present themselves. Firstly, the problem of completing the class so as to obtain a class of functions forming a complete Hilbert space and secondly, to decide (before effecting the completion of the class) if the complete class will possess a reproducing kernel.

A few remarks should be added here about the problem of the completion of a class of functions forming an incomplete Hilbert space. Consider such a class $F$. It is well known that to this class we can adjoin ideal elements which will be considered as the limits of Cauchy sequences in $F$, when such a limit is not available in $F$, and in such a way we obtain an abstract Hilbert space containing the class $F$ as a dense subset. This space, however, will not form a class of functions. In quite an arbitrary way we could realize the ideal elements to be adjoined to $F$ as functions so as to obtain a complete space formed by a linear class of functions, but, in general, this arbitrary manner of completion will destroy all the continuity properties between the values of the functions and the convergence in the space.

In this paper when we speak about the functional completion of an incomplete class of functions $F$, we mean a completion by adjunction of functions such that the value of a function $f$ of the completed class at a given point $y$ depends continuously on $f$ (as belonging to the Hilbert space) $\left({ }^{5}\right)$. From the existence theorem of reproducing kernels we deduce the fact that a completed class has a reproducing kernel. In this way the problem of functional completion and of the existence of a reproducing kernel in the complete class is merged into one problem. We shall prove here the following theorem:

Theorem. Consider a class of functions $F$ forming an incomplete Hilbert space. In order that there exist a functional completion of the class it is necessary and sufficient that $1^{\circ}$ for every fixed $y \in E$ the linear functional $f(y)$ defined in $F$ be bounded; $2^{\circ}$ for a Cauchy sequence $\left\{f_{m}\right\} \subset F$, the condition $f_{m}(y) \rightarrow 0$ for every $y$ implies $\left\|f_{m}\right\| \rightarrow 0$. If the functional completion is possible, it is unique.

Proof. That the first condition is necessary is immediately seen from the

(5) A more general functional completion was introduced in connection with the theory of pseudo-reproducing kernels (N. Aronszajn [5]). 
existence theorem of reproducing kernels, since the complete class would necessarily have such a kernel. The necessity of the second condition follows from the fact that a Cauchy sequence in $F$ is strongly convergent in the complete space to a function $f$, and the function $f$ is the limit of $f_{m}$ at every point $y$ of $E$. Consequently, $f=0$ and the norms of $f_{m}$ have to converge to the norm of $f$ which is equal to zero. To prove the sufficiency we proceed as follows: consider any Cauchy sequence $\left\{f_{n}\right\} \subset F$. For every fixed $y$ denote by $M_{y}$ the bound of the functional $f(y)$ so that

$$
f(y) \mid \leqq M_{y}\|f\| \text {. }
$$

Consequently,

$$
\left|f_{m}(y)-f_{n}(y)\right| \leqq M_{y}\left\|f_{m}-f_{n}\right\| .
$$

It follows that $\left\{f_{n}(y)\right\}$ is a Cauchy sequence of complex numbers, that is, it converges to a number which we shall denote by $f(y)$. In this way the Cauchy sequence $\left\{f_{n}\right\}$ defines a function $f$ to which it is convergent at every point of $E$.

Consider the class of all the functions $f$, limits of Cauchy sequences $\left\{f_{n}\right\} \subset F$. It is immediately seen that it is a linear class of functions, and that it contains the class $F$ (since the Cauchy sequence $\left\{f_{n}\right\}$ with $f_{n}=f \in F$ is obviously convergent to $f$ ). Consider, then, in the so-defined class $\bar{F}$, the norm

$$
\|f\|_{1}=\lim \left\|f_{n}\right\|
$$

for any Cauchy sequence $\left\{f_{n}\right\} \subset F$ converging to $f$ at every point $y$. This norm does not depend on the choice of the Cauchy sequence: in fact, if another sequence, $\left\{f_{n}^{\prime}\right\}$ converges to $f$ at every point $y$, then $f_{n}^{\prime}-f_{n}$ will be a Cauchy sequence converging to zero, and by the second condition the norms $\left\|f_{n}^{\prime}-f_{n}\right\|$ converge to zero.

Consequently,

$$
\left|\lim \left\|f_{n}^{\prime}\right\|-\lim \left\|f_{n}\right\|\right|=\lim \left|\left\|f_{n}^{\prime}\right\|-\left\|f_{n}\right\|\right| \leqq \lim \left\|f_{n}^{\prime}-f_{n}\right\|=0 .
$$

On the other hand, it is readily seen that $\|f\|_{1}^{2}$ is a quadratic positive form in the class $\bar{F}$; it is obviously 0 for $f=0$, and it is positive for $f \neq 0$ because of (1). This norm defines a scalar product in $\bar{F}$ satisfying all the required properties. It remains to be shown that $\bar{F}$ is complete and contains $F$ as a dense subspace.

The second assertion is immediately proved because $F \subset \bar{F}$. For elements of $F$, the norms \|\|$,\|\|_{1}$ coincide, and every function $f \in \bar{F}$ is, by definition, the limit of a Cauchy sequence $\left\{f_{n}\right\} \subset F$ everywhere in $E$. It follows that $f$ is a strong limit of $f_{n}$ in $\bar{F}$ since by (2)

$$
\lim _{n=\infty}\left\|f-f_{n}\right\|_{1}=\lim _{n=\infty} \lim _{m=\infty}\left\|f_{m}-f_{n}\right\|=0 .
$$


To prove the first assertion, that is, the completeness of $\bar{F}$, we shall consider any Cauchy sequence $\left\{f_{n}\right\} \subset \bar{F}$. Since $F$ is dense in $\bar{F}$, we can find a Cauchy sequence $\left\{f_{n}^{\prime}\right\} \subset F$ such that

$$
\lim \left\|f_{n}^{\prime}-f_{n}\right\|_{1}=0 .
$$

The Cauchy sequence $\left\{f_{n}^{\prime}\right\}$ converges to a function $f \in \bar{F}$. This convergence is meant at first as ordinary convergence everywhere in $E$, but the argument used above shows that the $f_{n}^{\prime}$ also converge strongly to $f$ in the space $\bar{F}$. It follows immediately that $f_{n}$ converges strongly to $f$. The uniqueness of the complete class is seen from the fact that in the completed class a function $f$ must necessarily be a strong limit of a Cauchy sequence $\left\{f_{n}\right\} \subset F$. Since a reproducing kernel must exist for the completed class, this implies that $f$ is a limit everywhere of the Cauchy sequence $\left\{f_{n}\right\}$ which means that it belongs to the above class $\bar{F}$. As the norm of $f$ has to be the limit of $\left\|f_{n}\right\|$ it necessarily coincides with $\|f\|_{1}$. It is also clear that every function $f$ of $\bar{F}$ must belong to the completed class. In summing up the above arguments we see that any functional completion of $F$ must coincide with $\bar{F}$ and have the same norm and scalar product as $\bar{F}$.

It should be stressed that the second condition cannot be excluded from our theorem. We shall demonstrate this by the following example:

Consider the unit circle $|z|<1$. Take there an infinite sequence of points $\left\{z_{n}\right\}$ such that

$$
\sum\left(1-\left|z_{n}\right|\right)<\infty .
$$

We shall denote by $E$ the set of all points $z_{n}$, and in $E$ we shall consider the class $F$ of all polynomials in $z$. It is obvious that the values of a polynomial cannot vanish everywhere in the set $E$ if the polynomial is not identically zero. Consequently the values of a polynomial on the set $E$ determine completely the polynomial. We define the norm for a function $f$ of the class $F$ by the formula

$$
\|f\|^{2}=\iint_{|z|<1}|p(z)|^{2} d x d y, \quad z=x+i y,
$$

where $p$ denotes the polynomial whose values on the set $E$ are given by the function $f$. We see that $F$ satisfies all requirements for a Hilbert space with the exception of completeness. The first condition of our theorem is satisfied but the second is not. To prove the last assertion we take the Blaschke function $\phi(z)$ corresponding to $\left\{z_{n}\right\}$. This function has the following properties

$$
\begin{array}{rr}
\phi\left(z_{n}\right)=0, & n=1,2,3, \cdots \\
|\phi(z)|<1 & \text { for }|z|<1 .
\end{array}
$$


The function $\phi(z)$ is a strong limit of polynomials $p_{k}(z)$ in the sense

$$
\lim \iint_{|z|<1}\left|\phi(z)-p_{k}(z)\right|^{2} d x d y=0 .
$$

Consequently the sequence $\left\{p_{k}\right\}$ is a Cauchy sequence in our class $F$ and the polynomials $p_{k}(z)$ converge at each point $z_{n}$ to $\phi\left(z_{n}\right)=0$, in spite of the fact that the norms $\left\|p_{k}\right\|$ converge to $\|\phi\|>0$.

This example also shows us the significance of condition $2^{\circ}$. We can say that if condition $2^{\circ}$ is not satisfied it means that the incomplete class is defined in too small a set. If we added a sequence of points $\left\{z_{n}^{\prime}\right\}$ of the unit circle with a limit point inside the circle to the set $E$ of our example, then the class of polynomials, considered on this enlarged set $E_{1}$ with the same norm as above, would satisfy condition $2^{\circ}$. There are infinitely many ways of enlarging the set $E$ where an incomplete class $F$ is defined so as to insure the fulfillment of condition $2^{\circ}$.

In the general case we can always proceed as follows. We can consider the abstract completion of the class $F$ by adjunction of ideal elements. This completion leads to an abstract Hilbert space $\mathfrak{S}$. To every element of $\mathfrak{S}$ there corresponds a well determined function $f(x)$ defined on the set (the limit of Cauchy sequences in $F$ converging to this element). But to different elements of $\mathfrak{S}$ there may correspond the same function $f$. The correspondence is linear and the functionals $f(y)$ are continuous in the whole $\mathfrak{S}$. To every point $y \in E$ there corresponds an element $h_{y} \in \mathfrak{S}$ such that $f(y)=\left(\bar{f}, h_{y}\right)$, where $\bar{f}$ is any element of $\mathfrak{S}$ corresponding to the function $f(x)$. As there are elements of $\mathfrak{S}$ different from the zero element and which correspond to the function identically zero on $E$, it is clear that the set of elements $h_{y}$ is not complete in the space $\mathfrak{F}$. (This is characteristic of the fact that condition $2^{\circ}$ is not satisfied.) To the set of elements $h_{y}$ we can then add an additional set of elements so as to obtain a complete set in $\mathfrak{S}$. This additional set will be denoted by $E^{\prime}$. We can then extend the functions of our class $F$ in the set $E+E^{\prime}$ by defining, for any element $h \in E^{\prime}, f(h)=(f, h)$. This class of functions, so extended in $E+E^{\prime}$, will then satisfy the second condition.

We shall complete this section by the following remark: It often happens that for the incomplete class $F$ a kernel $K(x, y)$ is known such that for every $y, K(x, y)$ as function of $x$ belongs to $F$ (or, even more generally, belongs to a Hilbert space containing $F$ as a subspace), this kernel having the reproducing property

$$
f(y)=(f(x), K(x, y))
$$$$
\text { for every } f \in F \text {. }
$$

It is immediately seen that the first condition of our theorem follows from this reproducing property so that it suffices to verify the second condition in order to be able to apply our theorem.

5. The restriction of a reproducing kernel. Consider a linear class $F$ of 
functions defined in the set $E$, forming a Hilbert space and possessing a r.k. $K(x, y) . K$ is a positive matrix. If we restrict the points $x, y$ to a subset $E_{1} \subset E, K$ will still be a positive matrix. This means that $K$ will correspond to a class $F_{1}$ of functions defined in $E_{1}$ with an adequate norm \|\|$_{1}$. We shall now determine this class $F_{1}$ and the corresponding norm.

THEOREM. If $K$ is the reproducing kernel of the class $F$ of functions defined in the set $E$ with the norm \|\| , then $K$ restricted to a subset $E_{1} \subset E$ is the reproducing kernel of the class $F_{1}$ of all restrictions of functions of $F$ to the subset $E_{1}$. For any such restriction, $f_{1} \in F_{1}$, the norm $\left\|f_{1}\right\|_{1}$ is the minimum of $\|f\|$ for all $f \in F$ whose restriction to $E_{1}$ is $f_{1}$.

Proof. Consider the closed linear subspace $F_{0} \subset F$ formed by all functions which vanish at every point of $E_{1}$. Take then the complementary subspace $F^{\prime}=F \ominus F_{0}$. Both $F_{0}$ and $F^{\prime}$ are closed linear subspaces of $F$ and possess reproducing kernels $K_{0}$ and $K^{\prime}$ such that

$$
K=K_{0}+K^{\prime} \text {. }
$$

Since $K_{0}(x, y)$, for every fixed $y$, belongs to $F_{0}$, it is vanishing for $x \in E_{1}$. Consequently,

$$
K(x, y)=K^{\prime}(x, y), \quad \text { for } x \in E_{1} \text {. }
$$

Consider now the class $F_{1}$ of all restrictions of $F$ to the set $E_{1}$. If two functions $f$ and $g$ from $F$ have the same restriction $f_{1}$ in $E_{1}, f-g$ vanishes on $E_{1}$ and so belongs to $F_{0}$. Conversely, if the difference belongs to $F_{0}, f$ and $g$ have the same restriction $f_{1}$ in $E_{1}$. It is then clear that all the functions $f \in F$ which have the same restriction $f_{1}$ in $E$ have a common projection $f_{1}^{\prime}$ on $F^{\prime}$ and that the restriction of $f_{1}^{\prime}$ in $E_{1}$ is equal also to $f_{1}$. It is also clear that among all these functions, $f, f_{1}^{\prime}$ is the one which has the smallest norm. Consequently by the definition of the theorem, we can write

$$
\left\|f_{1}\right\|_{1}=\left\|f_{1}^{\prime}\right\| \text {. }
$$

The correspondence between $f_{1} \in F_{1}$ and $f_{1}^{\prime} \in F^{\prime}$ obviously establishes a one-to-one isometric correspondence between the space $F_{1}$ with the norm \|\|$_{1}$ and the space $F^{\prime}$ with the norm \|\| .

In order to prove that for the class $F_{1}$ with the norm $\|\quad\|_{1}$ the reproducing kernel is given by $K$ restricted to $E_{1}$, we take any function $f_{1} \in F_{1}$ and consider the corresponding function $f_{1}^{\prime} \in F^{\prime}$. Then, for $y \in E_{1}, f_{1}(y)=f_{1}^{\prime}(y)$ $=\left(f_{1}^{\prime}(x), K^{\prime}(x, y)\right)$.

Since $K^{\prime}(x, y)$, for every $y$ belongs to $F^{\prime}$, we may now write $f_{1}(y)$ $=\left(f_{1}^{\prime} \mid(x), K^{\prime}(x, y)\right)=\left(f_{1}(x), K_{1}(x, y)\right)_{1}$, where $K_{1}(x, y)$ is the restriction of $K^{\prime}(x, y)$ (considered as function of $x$ ) to the set $E_{1}$.

By hermitian symmetry we obtain from (2) 


$$
K(x, y)=K^{\prime}(x, y), \quad \text { for every } y \in E_{1} .
$$

This shows that the restriction $K_{1}(x, y)$ of $K^{\prime}(x, y)$ coincides with the restriction of $K$ to the set $E_{1}$, which completes our proof.

The norm \|\|$_{1}$ is especially simple when the subclass $F_{0} \subset F$ is reduced to the zero function. In this case $F^{\prime}=F$, each function $f_{1} \in F_{1}$ is a restriction of one and only one function $f \in F$ (since every function of $F$ vanishing in $E_{1}$ vanishes identically everywhere in $E$ ), and therefore $\left\|f_{1}\right\|_{1}=\|f\|$ for the function $f$ having the restriction $f_{1}$.

6. Sum of reproducing kernels. Let $K_{1}(x, y)$ and $K_{2}(x, y)$ be reproducing kernels corresponding to the classes $F_{1}$ and $F_{2}$ of functions defined in the same set $E$ with the norms \|\|$_{1}$ and \|\|$_{2}$ respectively. $K_{1}$ and $K_{2}$ are positive matrices, and obviously $K=K_{1}+K_{2}$ is also a positive matrix.

We shall now find the class $F$ and the norm \|\| corresponding to $K$. Consider the Hilbert space $\mathfrak{S}$ formed by all couples $\left\{f_{1}, f_{2}\right\}$ with $f_{i} \in F_{i}$. The metric in this space will be given by the equation

$$
\left\|\left\{f_{1}, f_{2}\right\}\right\|^{2}=\left\|f_{1}\right\|_{1}^{2}+\left\|f_{2}\right\|_{2}^{2} \text {. }
$$

Consider the class $F_{0}$ of functions $f$ belonging at the same time to $F_{1}$ and $F_{2}$ ( $F_{0}$ may be reduced to the zero function). Denote by $\mathfrak{S}_{0}$ the set of all couples $\{f,-f\}$ for $f \in F_{0}$. It is clear that $\mathfrak{S}_{0}$ is a linear subspace of $\mathfrak{S}_{\mathrm{E}}$. It is a closed subspace. In fact if $\left\{f_{n},-f_{n}\right\} \rightarrow\left\{f^{\prime}, f^{\prime \prime}\right\}$ then $f_{n}$ converges strongly to $f^{\prime}$ in $F_{1}$, and $-f_{n}$ converges strongly to $f^{\prime \prime}$ in $F_{2}$. Consequently, $f_{n}$ converges in the ordinary sense to $f^{\prime}$ and $-f_{n}$ to $f^{\prime \prime}$, which means that $f^{\prime \prime}=-f^{\prime}$ and $f^{\prime}$ and $f^{\prime \prime}$ belong to $F_{0}$. $\mathfrak{S}_{0}$ being a closed linear subspace of $\mathfrak{S}$ we can consider the complementary subspace $\mathfrak{S}^{\prime}$ so that $\mathfrak{S}=\mathfrak{S}_{0} \oplus \mathfrak{S}^{\prime}$. To every element of $\mathfrak{S}$ : $\left\{f^{\prime}, f^{\prime \prime}\right\}$ there corresponds the function $f(x)=f^{\prime}(x)+f^{\prime \prime}(x)$. This is obviously a linear correspondence transforming the space $\mathfrak{S}$ into a linear class of functions $F$. The elements of $\mathfrak{S}$ which are transformed by this correspondence into the zero function are clearly the elements of $\mathfrak{S}_{0}$. Consequently, this correspondence transforms $\mathfrak{S E}^{\prime}$ in a one-to-one way into $F$. The inverse correspondence transforms every function $f \in F$ into an element $\left\{g^{\prime}(f), g^{\prime \prime}(f)\right\}$ of $\mathfrak{S}^{\prime}$. We define the metric in $F$ by the equation

$$
\|f\|^{2}=\left\|\left\{g^{\prime}(f), g^{\prime \prime}(f)\right\}\right\|^{2}=\left\|g^{\prime}(f)\right\|_{1}^{2}+\left\|g^{\prime \prime}(f)\right\|_{2 \cdot}^{2},
$$

Our assertion will be that to the class $F$ with the above-defined norm there corresponds the reproducing kernel $K=K_{1}+K_{2}$. To prove this assertion we remark:

(1) $K(x, y)$ as a function of $x$, for $y$ fixed, belongs to $F$. Namely, it corresponds to the element $\left\{K_{1}(x, y), K_{2}(x, y)\right\} \in \mathfrak{S}$.

(2) Denote for fixed $y, K^{\prime}(x, y)=g^{\prime}(K(x, y))$ and $K^{\prime \prime}(x, y)=g^{\prime \prime}(K(x, y))$. For a function $f \in F$, we write $f^{\prime}=g^{\prime}(f), f^{\prime \prime}=g^{\prime \prime}(f)$. Consequently, $f(y)=f^{\prime}(y)$ $+f^{\prime \prime}(y), K^{\prime}(x, y)+K^{\prime \prime}(x, y)=K(x, y)=K_{1}(x, y)+K_{2}(x, y)$, and thus $K^{\prime \prime}(x, y)$ $-K_{2}(x, y)=-\left[K^{\prime}(x, y)-K_{1}(x, y)\right]$, so that the element $\left\{K_{1}(x, y)-K^{\prime}(x, y)\right.$, 
$\left.K_{2}(x, y)-K^{\prime \prime}(x, y)\right\} \in \mathfrak{S}_{0}$. Hence

$$
\begin{aligned}
f(y)= & f^{\prime}(y)+f^{\prime \prime}(y)=\left(f^{\prime}(x), K_{1}(x, y)\right)_{1}+\left(f^{\prime \prime}(x), K_{2}(x, y)\right)_{2} \\
= & \left(\left\{f^{\prime}, f^{\prime \prime}\right\},\left\{K_{1}(x, y), K_{2}(x, y)\right\}\right) \\
= & \left(\left\{f^{\prime}, f^{\prime \prime}\right\},\left\{K^{\prime}(x, y), K^{\prime \prime}(x, y)\right\}\right) \\
& +\left(\left\{f^{\prime}, f^{\prime \prime}\right\},\left\{K_{1}(x, y)-K^{\prime}(x, y), K_{2}(x, y)-K^{\prime \prime}(x, y)\right\}\right) .
\end{aligned}
$$

The last scalar product equals zero since the element $\left\{f^{\prime}, f^{\prime \prime}\right\} \in \mathfrak{S}^{\prime}$ and the element $\left\{K_{1}(x, y)-K^{\prime}(x, y), K_{2}(x, y)-K^{\prime \prime}(x, y)\right\} \in \mathfrak{S}_{0}$. The first scalar product in the last member is, by our definition, equal to $(f(x), K(x, y))$ which proves the reproducing property of the kernel $K(x, y)$.

We can characterize the class $F$ as the class of all functions $f(x)=f_{1}(x)$ $+f_{2}(x)$ with $f_{i} \in F_{i}$. In order to define the corresponding norm without passage through the auxiliary space, we consider for every $f \in F$ all possible decompositions $f=f_{1}+f_{2}$ with $f_{i} \in F_{i}$. For each such decomposition we consider the sum $\left\|f_{1}\right\|_{1}^{2}+\left\|f_{2}\right\|_{2}^{2}$. $\|f\|$ will then be defined by

$$
\|f\|^{2}=\min \left[\left\|f_{1}\right\|_{1}^{2}+\left\|f_{2}\right\|_{2}^{2}\right]
$$

for all the decompositions of $f$. To prove the equivalence of this definition and the previous one we have only to remember that $f(x)$ corresponds to the element $\left\{f_{1}, f_{2}\right\} \in \mathfrak{S}$ and also that $f$ corresponds to $\left\{g^{\prime}(f), g^{\prime \prime}(f)\right\} \in \mathfrak{S}^{\prime}$, that is, $f=f_{1}+f_{2}=g^{\prime}(f)+g^{\prime \prime}(f)$. Consequently,

$$
f_{2}-g^{\prime \prime}(f)=-\left[f_{1}-g^{\prime}(f)\right]
$$

so that $\left\{f_{1}-g^{\prime}(f), f_{2}-g^{\prime \prime}(f)\right\} \in \mathfrak{S}_{0}$ and

$\left\|f_{1}\right\|_{1}^{2}+\left\|f_{2}\right\|_{2}^{2}=\left\|\left\{f_{1}, f_{2}\right\}\right\|^{2}=\left\|\left\{g^{\prime}(f), g^{\prime \prime}(f)\right\}\right\|^{2}+\left\|\left\{f_{1}-g^{\prime}(f), f_{2}-g^{\prime \prime}(f)\right\}\right\|^{2}$,

and this expression will obviously get the minimal value if and only if $f_{1}=g^{\prime}(f), f_{2}=g^{\prime \prime}(f)$ and its value is then given by

$$
\left\|\left\{g^{\prime}(f), g^{\prime \prime}(f)\right\}\right\|^{2} \text {, }
$$

which by our previous definition is $\|f\|^{2}$.

Summing up, we may write the following theorem:

THEOREM. If $K_{i}(x, y)$ is the reproducing kernel of the class $F_{i}$ with the norm \|\|$_{i}$, then $K(x, y)=K_{1}(x, y)+K_{2}(x, y)$ is the reproducing kernel of the class $F$ of all functions $f=f_{1}+f_{2}$ with $f_{i} \in F_{i}$, and with the norm defined by

$$
\|f\|^{2}=\min \left[\left\|f_{1}\right\|_{1}^{2}+\left\|f_{2}\right\|_{2}^{2}\right] \text {, }
$$

the minimum taken for all the decompositions $f=f_{1}+f_{2}$ with $f_{i} \in F_{i}\left({ }^{6}\right)$.

It is easy to see how this theorem can be extended to the case where

(6) This theorem was found by R. Godement [1] in the case of a positive definite function in a group. 
$K(x, y)=\sum_{i=1}^{n} K_{i}(x, y)$. A particularly simple case presents itself when the classes $F_{1}$ and $F_{2}$ have no function besides zero in common. The norm in $F$ is then given simply by $\|f\|^{2}=\left\|f_{1}\right\|_{1}^{2}+\left\|f_{2}\right\|_{2}^{2}$.

In this case (and only in this case) $F_{1}$ and $F_{2}$ are complementary closed subspaces of $F$.

If we denote by $\bar{F}$ the class of all conjugate functions of functions of a class $F$, then the kernel of $\bar{F}$ is clearly $K_{1}(x, y)=\overline{K(x, y)}=K(y, x)$ ( $K$ is the kernel of $F$ and in $\bar{F}$ the scalar product $(\bar{f}, \bar{g})_{1}$ is given by $(g, f)$, the norm $\|\bar{f}\|_{1}$ by $\left.\|f\|\right)$. Consequently $\operatorname{Re} K(x, y)=2^{-1}(K(x, y)+K(y, x))$ is the reproducing kernel of the class $F_{0}$ of all sums $f+\bar{g}$, for $f$ and $g$ in $F$ with the norm given by

$$
\|\phi\|_{0}^{2}=2 \min \left[\|f\|^{2}+\|g\|^{2}\right],
$$

the minimum taken for all decompositions $\phi=f+\bar{g}, f$ and $g$ in $F$.

If $F$ is a complex space corresponding to a real space, that is, if $F=\bar{F}$ and $\|f\|=\|\vec{f}\|$ (see $\S 1$ ), it is clear that $F_{0}=F$ and $\|f\|_{0}=\|f\|$. Consequently the kernel $K=\operatorname{Re} K$ is real and this property characterizes the kernal $K$ corresponding to a real space.

7. Difference of reproducing kernels. For two positive matrices, $K_{1}(x, y)$ and $K(x, y)$, we shall write

$$
K_{1} \ll K
$$

if $K(x, y)-K_{1}(x, y)$ is also a p. matrix.

From $K_{1} \ll K_{2} \ll K_{3}$, it follows clearly that $K_{1} \ll K_{3}$. On the other hand, if $K_{1} \ll K_{2}$ and $K_{2} \ll K_{1}$, it follows that $K_{1}=K_{2}$. In fact, we then have $K_{2}(x, x)$ $-K_{1}(x, x) \geqq 0$ and also $K_{1}(x, x)-K_{2}(x, x) \geqq 0$, which means $K_{2}(x, x)-K_{1}(x, x)$ $=0$. Further, by the property of positive matrices,

$$
\left|K_{2}(x, y)-K_{1}(x, y)\right|^{2} \leqq\left[K_{2}(x, x)-K_{1}(x, x)\right]\left[K_{2}(y, y)-K_{1}(y, y)\right]=0,
$$

so that $K_{2}(x, y)=K_{1}(x, y)$ for every $x, y$ in $E$. Thus we see that the symbol $\ll$ establishes a partial ordering in the class of all positive matrices.

THEOREM I. If $K$ and $K_{1}$ are the r.k.'s of the classes $F$ and $F_{1}$ with the norms \|\|$,\|\|_{1}$, and if $K_{1} \ll K$, then $F_{1} \subset F$, and $\left\|f_{1}\right\|_{1} \geqq\left\|f_{1}\right\|$ for every $f_{1} \in F_{1}$.

Proof. $K_{1} \ll K$ means that $K_{2}(x, y)=K(x, y)-K_{1}(x, y)$ is a positive mat:-ix. Consider the class of functions $F_{2}$ and the norm \|\|$_{2}$ corresponding to $\ddot{n}_{2}$. As $K=K_{1}+K_{2}$ we know by the theorem of $\S 6$ that $F$ is the class of all functions of the form $f_{1}(x)+f_{2}(x)$ with $f_{1} \in F_{1}$ and $f_{2} \in F_{2}$. In particular, when $f_{2}=0$, the class $F$ contains all functions $f_{1} \in F_{1}$ so that $F_{1} \subset F$. On the other hand, in $F$ we have, by the same theorem,

$$
\left\|f_{1}\right\|^{2}=\min \left[\left\|f_{1}^{\prime}\right\|_{1}^{2}+\left\|f_{2}^{\prime}\right\|_{2}^{2}\right]
$$

for all decompositions $f_{1}=f_{1}^{\prime}+f_{2}^{\prime}$, with $f_{1}^{\prime} \in F_{1}$ and $f_{2}^{\prime} \in F_{2}$. In particular, for 
the decomposition $f_{1}=f_{1}+0$, we obtain

$$
\left\|f_{1}\right\|^{2} \leqq\left\|f_{1}\right\|_{1}^{2}
$$

which achieves the proof.

THEOREM II. If $K$ is the r.k. of the class $F$ with the norm \|\| , and if the linear class $F_{1} \subset F$ forms a Hilbert space with the norm \|\|$_{1}$, such that $\left\|f_{1}\right\|_{1}$ $\geqq\left\|f_{1}\right\|$ for every $f_{1} \in F_{1}$, then the class $F_{1}$ possesses a reproducing kernel $K_{1}$ satisfying $K_{1} \ll K$.

Proof. The existence of the kernel for $F$ involves the existence of constants $M_{y}$ such that $|f(y)| \leqq M_{y}\|f\|$ for all $f \in F$. In particular, for $f_{1} \in F_{1} \subset F$, we get

$$
\left|f_{1}(y)\right| \leqq M_{y}\left\|f_{1}\right\| \leqq M_{y}\left\|f_{1}\right\|_{1}
$$

which proves the existence of the kernel $K_{1}$ of $F_{1}$.

Let us now introduce an operator $L$ in the space $F$, transforming the space $F$ into the space $F_{1}$ and satisfying the equation

$$
\left(f_{1}, f\right)=\left(f_{1}, L f\right)_{1}, \quad \text { for every } f_{1} \in F_{1} .
$$

The existence and unicity of $L f$ is proved in the following way: $\left(f_{1}, f\right)$, for fixed $f$, is a linear continuous functional of $f_{1}$ in the space $F$. A fortiori, it is a similar functional in the space $F_{1}$. As such, it is representable as a scalar product in $F_{1}$ of $f_{1}$ with a uniquely determined element $L f$ of $F_{1}$.

The operator $L$ is everywhere defined in $F$, linear, symmetric, positive, and bounded with a bound not greater than 1 . It is clear that $L$ is everywhere defined and linear. It is symmetric because for any two functions $f, f^{\prime}$ of $F$, $\left(L f, f^{\prime}\right)=\left(L f, L f^{\prime}\right)_{1}=\overline{\left(L f^{\prime}, L f\right)_{1}}=\overline{\left(L f^{\prime}, f\right)}=\left(f, L f^{\prime}\right)$. It is positive because $(L f, f)=(L f, L f)_{1} \geqq 0$. It is bounded with a bound not greater than 1 because $(L f, f)=(L f, L f)_{1}=\|L f\|_{1}^{2} \geqq\|L f\|^{2}$. Consequently, $\|L f\|^{2} \leqq(L f, f) \leqq\|L f\| \cdot\|f\|$, and thus $\|L f\| \leqq\|f\|$.

Consider now the operator $I-L$ ( $I$ being the identical operator). This operator clearly possesses the same properties as those enumerated above for $L$. Therefore there exists a symmetric, bounded square root $L^{\prime}$ of this operator. (In general there will be infinitely many $L^{\prime}$ available and we choose any one of them.) Hence

$$
L^{\prime 2}=I-L \text {. }
$$

We define $F_{2}$ as the class of all functions $f_{2}=L^{\prime} f$ for $f \in F$. Denote by $F_{0}$ the closed linear subspace of $F$ transformed by $L^{\prime}$ into 0 , and by $F^{\prime}$ the complementary space $F \ominus F_{0}$. The functions of $F_{0}$ are also characterized by the fact that $L^{\prime 2} f=0$ (from $L^{\prime 2} f=0$ it follows that $\left(L^{\prime 2} f, f\right)=\left(L^{\prime} f, L^{\prime} f\right)=\left\|L^{\prime} f\right\|^{2}$ $=0$ ) which is equivalent to $f=L f$.

Now denote by $P^{\prime}$ the projection on $F^{\prime}$. Every two functions $f, g$ of $F$ transformed by $L^{\prime}$ into the same function $f_{2}$ of $F_{2}$ differ by a function belong- 
ing to $F_{0}$. Consequently, they have the same projection on $F^{\prime}$. It is also clear that the class $F^{\prime}$ is transformed by $L^{\prime}$ on $F_{2}$ in a one-to-one way. We remark further that

$$
F_{2} \subset F^{\prime}
$$

since for $f_{0} \in F_{0},\left(f_{0}, L^{\prime} f\right)=\left(L^{\prime} f_{0}, f\right)=(0, f)=0$. In the class $F_{2}$ we introduce the norm \|\|$_{2}$ by the equation

$$
\left\|f_{2}\right\|_{2}=\left\|P^{\prime} f\right\|, \quad \text { for any } f \text { with } f_{2}=L^{\prime} f .
$$

With this metric the class $F_{2}$ is isometric to the subspace $F^{\prime} \subset F$ (the isometry being given by the transformation $L^{\prime}$ ). It follows that $F_{2}$ is a complete Hilbert space and we shall show that the class $F_{2}$ with the norm \|\|$_{2}$ admits as reproducing kernel the difference $K(x, y)-K_{1}(x, y)$. To this effect we remark firstly that the operator $L$ is given by the formula

$$
f_{1}(y) \equiv L f=\left(f, K_{1}(x, y)\right) .
$$

In fact, since $L f \in F_{1}, f_{1}(y)=\left(f_{1}(x), K_{1}(x, y)\right)_{1}=\left(f(x), K_{1}(x, y)\right)$. Consequently for any fixed $z$, the operator $L$ applied to $K(x, z)$ gives the function $L K(x, z)=K_{1}(y, z)$. If follows that the operator $I-L=L^{\prime 2}$ transforms $K(x, z)$ into $L^{\prime 2} K(x, z)=K(y, z)-K_{1}(y, z)$. This formula proves, firstly, that $K(x, z)$ $-K_{1}(x, z)$ as a function of $x$ belongs to $F_{2}$, since it is the transform of $L^{\prime} K(x, z)$ by $L^{\prime}$. Secondly, we prove the reproducing property for any $f_{2} \in F_{2}$ :

$$
\begin{aligned}
f_{2}(y) & =\left(f_{2}(x), K(x, y)\right)=\left(L^{\prime} f, K(x, y)\right)=\left(f, L^{\prime} K(x, y)\right) \\
& =\left(P^{\prime} f, P^{\prime} L^{\prime} K(x, y)\right)=\left(L^{\prime} f, L^{\prime} L^{\prime} K(x, y)\right)_{2} \\
& =\left(f_{2}(x), K(x, y)-K_{1}(x, y)\right)_{2} .
\end{aligned}
$$

In these transformations we took $f$ as any function of $F$ such that $f_{2}=L^{\prime} f$ and we used the property $L^{\prime} K(x, y) \in F_{2} \subset F^{\prime}$. This finishes the proof of our theorem.

The proof of Theorem II has established even more than the theorem announced: namely, it gives us the construction of the class $F_{2}$ and the metric \|\|$_{2}$ for which the difference $K-K_{1}$ is the reproducing kernel. Let us summarize this in a separate theorem.

Theorem III. Under the hypotheses of Theorem II, the class $F_{2}$ and the norm \|\|$_{2}$ corresponding to the kernel $K_{2}=K-K_{1}$ are defined as follows: the equation $f_{1}(y) \equiv L f=\left(f(x), K_{1}(x, y)\right)_{x}$ defines in $F$ a positive operator with bound not greater than 1 , transforming $F$ into $F_{1} \subset F$. We take any symmetric square root $L^{\prime}=(I-L)^{1 / 2} . F_{2}$ is the class of all transforms $L^{\prime} f$ for $f \in F$. Let $F_{0}$ be the closed linear subspace of all functions $f \in F$ with $f=L f$ and let $F^{\prime}$ be $F \ominus F_{0} . L^{\prime}$ establishes a one-to-one correspondence between $F^{\prime}$ and $F_{2}$. The norm $\left\|f_{2}\right\|_{2}$ for $f_{2}=L^{\prime} f^{\prime}, f^{\prime} \in F^{\prime}$, is then given by 


$$
\left\|f_{2}\right\|_{2}=\left\|f^{\prime}\right\| \text {. }
$$

The construction of the class $F_{2}$ is somehow complicated because we need the square root $L^{\prime}$ of the operator $I-L$. However, there exists a much easier way of constructing an everywhere dense linear subclass of $F_{2}$ and the norm \|\|$_{2}$ in this subclass. Namely, we can consider the class $F_{2}^{\prime}$ of all transforms $(I-L) f=f-L f=L^{\prime 2} f$, of $f \in F$, by the operator $I-L$.

This clearly is a linear subclass of $F_{2}$. The norm \|\|$_{2}$ in $F_{2}^{\prime}$ can be defined as follows:

$$
\begin{aligned}
\left\|f_{2}^{\prime}\right\|_{2}^{2} & =\left\|L^{\prime} f\right\|^{2}=\left(L^{\prime} f, L^{\prime} f\right)=\left(f,{L^{\prime}}^{2} f\right)=(f, f-L f)=(f, f)-(f, L f) \\
& =(f, f)-(L f, L f)_{1} .
\end{aligned}
$$

In these transformations we considered $f_{2}^{\prime}=f-L f=L^{\prime 2} f$.

$F_{2}^{\prime}$ is everywhere dense in $F_{2}$ (in respect to the norm \|\|$_{2}$ ), otherwise we would have a function $f_{2}=L^{\prime} f^{\prime} \neq 0$ with $\left(L^{\prime} f^{\prime}, L^{\prime 2} g\right)_{2}=0$ for all $g \in F$. We can suppose here $f^{\prime} \in F^{\prime}$ so that $0=\left(L^{\prime} f^{\prime}, L^{\prime 2} g\right)_{2}=\left(f^{\prime}, L^{\prime} g\right)=\left(L^{\prime} f^{\prime}, g\right)$ whence $L^{\prime} f^{\prime}=0, f^{\prime} \in F_{0}$, which is impossible.

The simplest case is the one where $F_{2}^{\prime}=F_{2}$. By using the spectral decomposition, we can easily show that this case presents itself if, and only if, the zero is not a limit point of the spectrum of $L^{\prime}$, which is the equivalent of saying that 1 is not a limit point of the spectrum of $L$. In this case $F_{2}=F^{\prime}$. If $L$ has a bound $<1$, the spectrum does not contain 1 and the subspace $F^{\prime}$ coincides with the whole space $F$ so that $F_{2}=F$.

When $L$ is completely continuous, the only limit point of the spectrum of $L$ is zero, so that 1 is certainly not a limit point, and $F_{2}^{\prime}=F_{2}=F^{\prime}$.

We shall add still another theorem which results immediately from Theorem III.

Theorem IV. Let $K$ be the r.k. of class $F$. To every decomposition $K=K_{1}$ $+K_{2}$ in two p. matrices $K_{1}$ and $K_{2}$, there corresponds a decomposition of the identity operator $I$ in $F$ in two positive operators $L_{1}$ and $L_{2}, I=L_{1}+L_{2}$, given by

$$
L_{1} f(y)=\left(f(x), K_{1}(x, y)\right), \quad L_{2} f(y)=\left(f(x), K_{2}(x, y)\right),
$$

such that if $L_{1}^{1 / 2}$ and $L_{2}^{1 / 2}$ denote any symmetric square roots of $L_{1}$ and $L_{2}$, the classes $F_{1}$ and $F_{2}$ of all transforms $L_{1}^{1 / 2}$ fand $L_{2}^{1 / 2}$ frespectively, $f \in F$, correspond to the kernels $K_{1}$ and $K_{2}$. If $F_{i 0}, i=1,2$, is the class of all $f \in F$ with $L_{i} f=0$, and if $F_{i}^{\prime \prime}=F \ominus F_{i 0}$, then $L_{i}^{1 / 2}$ establishes a one-to-one correspondence between $F_{i}^{\prime \prime}$ and $F_{i}$ and the norm \|\|${ }_{i}$ in $F_{i}$ is given by $\left\|L_{i}^{1 / 2} f\right\|_{i}=\|f\|$ for every $f \in F_{i}^{\prime \prime}$.

Conversely, to each decomposition $I=L_{1}+L_{2}$ in two positive operators there correspond classes $F_{i}$ with norms \|\|$_{i}$ defined as above. The corresponding $r . k . ' s$ $K_{i}$ are defined by $K_{i}(x, y)=L_{i} K(x, y)$ and satisfy the equation $K=K_{1}+K_{2}$.

8. Product of reproducing kernels. Consider two positive matrices $K_{1}$ 
and $K_{2}$ defined in a set $E$. Using a classical result of I. Schur concerning finite matrices, it is easy to prove that their product $K_{1} \cdot K_{2}$ is also a positive matrix. We shall prove this theorem, constructing at the same time the class $F$ and the norm \|\| corresponding to the matrix $K=K_{1} \cdot K_{2}\left({ }^{7}\right)$. To this effect, we consider the class $F_{i}$ and the norm $\|\quad\|_{i}$ corresponding to $K_{i}$ and form the direct product $F^{\prime}$ of the Hilbert spaces $F_{1}$ and $F_{2}, F^{\prime \prime}=F_{1} \otimes F_{2}\left({ }^{8}\right)$. We construct this direct product in the following manner: We form the product set $E^{\prime}=E \times E$ of all couples of points $\left\{x_{1}, x_{2}\right\}, x_{i} \in E$. In the set $E^{\prime}$ consider the class of all functions $f^{\prime}\left(x_{1}, x_{2}\right)$ representable in the form:

$$
f^{\prime}\left(x_{1}, x_{2}\right)=\sum_{k=1}^{n} f_{1}^{(k)}\left(x_{1}\right) f_{2}^{(k)}\left(x_{2}\right)
$$

with $f_{1}^{(k)} \in F_{1}$ and $f_{2}^{(k)} \in F_{2}$. As scalar product of two such functions we define:

$$
\left(f^{\prime}, g^{\prime}\right)^{\prime}=\sum_{k=1}^{n} \sum_{l=1}^{m}\left(f_{1}^{(k)}, g_{1}^{(l)}\right)_{1}\left(f_{2}^{(k)}, g_{2}^{(l)}\right)_{2},
$$

where $m$ is the number of terms in the representation of $g^{\prime}$. The same function $f^{\prime}$ may admit of many different representations of type (1). The scalar product, $\left(f^{\prime}, g^{\prime}\right)^{\prime}$, is independent of the particular representation chosen for $f^{\prime}$ and $g^{\prime}$. In fact, we see immediately from (2) that

$$
\left(f^{\prime}, g^{\prime}\right)^{\prime}=\sum_{l=1}^{m}\left(\left(f^{\prime}\left(x_{1}, x_{2}\right), g_{1}^{(l)}\left(x_{1}\right)\right)_{1}, g_{2}^{(l)}(x)\right)_{2}
$$

which proves that $\left(f^{\prime}, g^{\prime}\right)$ is independent of the particular representation of $f^{\prime}$. In a similar way we prove that it is independent of the particular representation of $g^{\prime}$. We still have to prove that $\left(f^{\prime}, g^{\prime}\right)^{\prime}$ satisfies all the requirements for the scalar product. It is clearly seen that it is a bilinear hermitian form in $f^{\prime}, g^{\prime}$ and it remains to be proved that $\left(f^{\prime}, f^{\prime}\right)^{\prime} \geqq 0$ and is equal to zero only when $f^{\prime}=0$. In order to prove this, we take any representation of $f^{\prime}$ of type (1) and orthonormalize the sequences $\left\{f_{1}^{(\mathbf{k})}\right\}$ and $\left\{f_{2}^{(\mathbf{k})}\right\}$ in the spaces $F_{1}$ and $F_{2}$ respectively. Denote by $\left\{f_{1}^{(k, 1)}\right\}$ and $\left\{f_{2}^{(l, 1)}\right\}$ the orthonormalized sequences, where $k=1,2, \cdots, n_{1}, l=1,2, \cdots, n_{2}$.

Every function $f_{i}^{(k)}$ is then a linear combination of the orthonormal functions $f_{i}^{\left(\mathbf{k}^{\prime}, 1\right)}$ so that we obtain a representation for $f^{\prime}$ as a double series

$$
f^{\prime}\left(x_{1}, x_{2}\right)=\sum_{k=1}^{n_{1}} \sum_{l=1}^{n_{2}} \alpha_{k, l} f_{1}^{(k, 1)} f_{2}^{(l, 1)} .
$$

We then obtain for $\left(f^{\prime}, f^{\prime}\right)^{\prime}$ the following expression

( 7 ) The idea of the proof was arrived at independently by R. Godement and the author. Godement applied it only to positive definite functions.

${ }^{(8)}$ For the notion of direct product of abstract Hilbert spaces see J. v. Neumann and F. J. Murray [1]. 
(5)

$$
\begin{aligned}
\left(f^{\prime}, f^{\prime}\right) & =\sum_{k=1}^{n_{1}} \sum_{l=1}^{n_{2}} \sum_{k^{\prime}=1}^{n_{1}} \sum_{l^{\prime}=1}^{n_{2}} \alpha_{k, l} \bar{\alpha}_{k^{\prime}, l^{\prime}}\left(f_{1}^{(k, 1)}, f_{1}^{\left(k^{\prime}, 1\right)}\right)_{1}\left(f_{2}^{(l, 1)}, f_{2}^{\left(l^{\prime}, 1\right)}\right)_{2} \\
& =\sum_{k=1}^{n_{1}} \sum_{l=1}^{n_{2}}\left|\alpha_{k, l}\right|^{2} .
\end{aligned}
$$

It is clear from this representation that $\left(f^{\prime}, f^{\prime}\right) \geqq 0$ and equals zero only when all the $\alpha_{k, l}=0$, that is, when $f^{\prime}=0$. The class of all functions $f^{\prime}$ of type (1) does not yet form in general a Hilbert space because it may not be complete. To complete this class with respect to the norm \|\|$^{\prime}$, we consider a complete orthonormal sequence $\left\{g_{i}^{(\mathrm{k})}\right\}$ in the space $F_{i}, i=1,2$. It is obvious that the double sequence $\left\{g_{1}^{(k)}\left(x_{i}\right) \cdot g_{2}^{(l)}\left(x_{2}\right)\right\}$ is composed of functions of type (1) and is orthonormal in respect to the norm \|\|$^{\prime}$. Consider then, all the functions $g^{\prime}$ of the form

$$
g^{\prime}\left(x_{1}, x_{2}\right)=\sum_{k=1}^{\infty} \sum_{l=1}^{\infty} \alpha_{k, l} g_{1}^{(k)}\left(x_{1}\right) g_{2}^{(l)}\left(x_{2}\right)
$$

with

$$
\left(g^{\prime}, g^{\prime}\right)^{\prime}=\sum_{k=1}^{\infty} \sum_{l=1}^{\infty}\left|\alpha_{k, l}\right|^{2}<\infty .
$$

It is clear that any finite sum of type (6) is also of type (1) and that the norm \|\|$^{\prime}$ for such finite sums coincides with the norm introduced in (7). We prove firstly that every sum of type (6) is absolutely convergent for every $x_{1}, x_{2}$. In fact, as the class $F_{1}$ possesses the reproducing kernel $K_{1}$, in view of (7) we have

$$
\sum_{k=1}^{\infty}\left|\alpha_{k, l}\right|\left|g_{1}^{(k)}\left(x_{1}\right)\right| \leqq\left[K_{1}\left(x_{1}, x_{1}\right)\right]^{1 / 2}\left[\sum_{k=1}^{\infty}\left|\alpha_{k, l}\right|^{2}\right]^{1 / 2} .
$$

Then,

$$
\begin{aligned}
& \sum_{k=1}^{\infty} \sum_{l=1}^{\infty}\left|\alpha_{k, l}\right|\left|g_{1}^{(k)}\left(x_{1}\right)\right|\left|g_{2}^{(l)}\left(x_{2}\right)\right| \\
& \leqq \sum_{l=1}^{\infty}\left|g_{2}^{(l)}\left(x_{2}\right)\right|\left[K_{1}\left(x_{1}, x_{1}\right)\right]^{1 / 2}\left[\sum_{k=1}^{\infty}\left|\alpha_{k, l}\right|^{2}\right]^{1 / 2} \\
& \leqq\left[K_{1}\left(x_{1}, x_{1}\right)\right]^{1 / 2}\left[K_{2}\left(x_{2}, x_{2}\right)\right]^{1 / 2} \cdot\left[\sum_{k=1}^{\infty} \sum_{l=1}^{\infty}\left|\alpha_{k, l}\right|^{2}\right]^{1 / 2}
\end{aligned}
$$

since the space $F_{2}$ possesses the reproducing kernel $K_{2}$.

The class of functions $g^{\prime}$ of the form (6) clearly forms a complete Hilbert space, isomorphic with the space of double sequences $\left\{\alpha_{k, l}\right\}$ satisfying (7). The inequality (8) gives us further, for a function $g^{\prime}$ of type (6), 


$$
\left|g^{\prime}\left(y_{1}, y_{2}\right)\right|<K_{1}\left(y_{1}, y_{1}\right)^{1 / 2} K_{2}\left(y_{2}, y_{2}\right)^{1 / 2}\left\|g^{\prime}\right\|^{\prime},
$$

which means that the space of functions of type (6) possesses a reproducing kernel.

It remains to be proved that this space is the completion of the class of all functions $f^{\prime}$ of type (1) with the norm \|\|$\prime$. As already the finite sums of type (6) are everywhere dense in the space of all functions of type (6) it is sufficient to prove that every function of type (1) is of type (6). For this, it is enough to prove that every function of type (1) may be approximated as closely as we wish (in respect to the norm \|\|$^{\prime}$ ) by finite sums of type (6). Let us consider a representation (1) of the function $f^{\prime}$. We can approximate every $f_{i}^{(k)}$ by a finite linear combination $h_{i}^{(k)}$ of functions $g_{i}^{(l)}$ so that $\left\|h_{i}^{(k)}\right\|_{i}$ $\leqq\left\|f_{i}^{(k)}\right\|_{i},\left\|f_{i}^{(k)}-h_{i}^{(k)}\right\|_{i} \leqq \epsilon$. Before we proceed further, we shall prove for every function $f^{\prime}$ and any of its representations (1) the inequality

$$
\left\|f^{\prime}\right\|^{\prime} \leqq \sum_{k=1}^{n}\left\|f_{1}^{(k)}\right\|_{1} \cdot\left\|f_{2}^{(k)}\right\|_{2}
$$

In fact,

$$
\begin{aligned}
\left\|f^{\prime}\right\|^{\prime 2}=\left(f^{\prime}, f^{\prime}\right)^{\prime} & =\sum_{k=1}^{n} \sum_{l=1}^{n}\left(f_{1}^{(k)}, f_{1}^{(l)}\right)_{1}\left(f_{2}^{(k)}, f_{2}^{(l)}\right)_{2} \\
& \leqq \sum_{k=1}^{n} \sum_{l=1}^{n}\left\|f_{1}^{(k)}\right\|\left\|_{1}\right\| f_{1}^{(l)}\left\|_{1} \cdot\right\| f_{2}^{(k)}\left\|_{2}\right\| f_{2}^{(l)} \|_{2} \\
& =\left[\sum_{k=1}^{n}\left\|f_{1}^{(k)}\right\|_{1}\left\|f_{2}^{(k)}\right\|_{2}\right]^{2}
\end{aligned}
$$

Continuing with the proof of the approximation we consider the functions

$$
\begin{aligned}
& h^{\prime}\left(x_{1}, x_{2}\right)=\sum_{k=1}^{n} h_{1}^{(k)}\left(x_{1}\right) f_{2}^{(k)}\left(x_{2}\right), \\
& g^{\prime}\left(x_{1}, x_{2}\right)=\sum_{k=1}^{n} h_{1}^{(k)}\left(x_{1}\right) h_{2}^{(k)}\left(x_{2}\right) .
\end{aligned}
$$

It is clear that $h^{\prime}$ is of type (1) and that $g^{\prime}$ is at the same time of type (1) and (6), which can be seen by developing the functions $h_{i}^{(k)}$ as linear combinations of $g_{i}^{(l)}$. Denoting by $M$ the maximum of all $\left\|f_{i}^{(k)}\right\|_{i}$, we obtain

$$
\begin{aligned}
\left\|f^{\prime}-g^{\prime}\right\|^{\prime} & \leqq\left\|f^{\prime}-h^{\prime}\right\|^{\prime}+\left\|h^{\prime}-g^{\prime}\right\|^{\prime} \\
\left\|f^{\prime}-h^{\prime}\right\|^{\prime} & =\sum_{k=1}^{n}\left\|\left(f_{1}^{(k)}\left(x_{1}\right)-h_{1}^{(k)}\left(x_{1}\right)\right) f_{2}^{(k)}\left(x_{2}\right)\right\|^{\prime} \\
& \leqq \sum_{k=1}^{n}\left\|f_{1}^{(k)}-h_{1}^{(k)}\right\|_{1} \cdot\left\|f_{2}^{(k)}\right\|_{2} \leqq \sum_{k=1}^{n} M \epsilon=n M \epsilon
\end{aligned}
$$




$$
\begin{aligned}
\left\|h^{\prime}-g^{\prime}\right\|^{\prime} & =\| \sum_{k=1}^{n} h_{1}^{(k)}\left(x_{1}\right)\left(\left(f_{2}^{(k)}\left(x_{2}\right)-h_{2}^{(k)}\left(x_{2}\right)\right) \|^{\prime}\right. \\
& \leqq \sum_{k=1}^{n}\left\|h_{1}^{(k)}\right\|_{1} \cdot\left\|f_{2}^{(k)}-h_{2}^{(k)}\right\|_{2} \leqq \sum_{k=1}^{n} M \epsilon=n M \epsilon .
\end{aligned}
$$

Finally, we obtain

$$
\left\|f^{\prime}-g^{\prime}\right\|^{\prime} \leqq 2 n M \epsilon
$$

which proves our assertion.

The class of all functions of type (6) with the norm given by (7) forms the direct product $F^{\prime}=F_{1} \otimes F_{2}$. As it is obtained by functional completion of the class of functions of type (1), this class being independent of the choice of orthogonal systems $\left\{g_{1}^{(k)}\right\}$ and $\left\{g_{2}^{(k)}\right\}$, the class $F^{\prime}$ is also independent of the choice of these systems (see the uniqueness of functional completion in $\$ 4$ ).

We shall now prove the following theorem:

TheOREM I. The direct product $F^{\prime}=F_{1} \otimes F_{2}$ possesses the reproducing kernel $K^{\prime}\left(x_{1}, x_{2}, y_{1}, y_{2}\right)=K_{1}\left(x_{1}, y_{1}\right) K_{2}\left(x_{2}, y_{2}\right)$.

The proof is immediate. Firstly, as a function of $x_{1}, x_{2}, K^{\prime}$ is of the form (1) and so belongs to $F^{\prime}$. Secondly, for any function $g^{\prime}$ of the form (6) we have

$$
\begin{aligned}
g^{\prime}\left(y_{1}, y_{2}\right) & =\sum \sum \alpha_{k, l}\left(g_{1}^{(k)}\left(x_{1}\right), K_{1}\left(x_{1}, y_{1}\right)\right)_{1}\left(g_{2}^{(l)}\left(x_{2}\right), K_{2}\left(x_{2}, y_{2}\right)\right)_{2} \\
& =\left(g^{\prime}\left(x_{1}, x_{2}\right), K^{\prime}\left(x_{1}, x_{2}, y_{1}, y_{2}\right)\right)^{\prime}
\end{aligned}
$$

which completes the proof.

From Theorem I we see immediately that the kernel $K(x, y)$ $=K_{1}(x, y) K_{2}(x, y)$ is a p. matrix as the restriction of the kernel $K^{\prime}\left(x_{1}, x_{2}, y_{1}, y_{2}\right)$ to the subset $E_{1} \subset E^{\prime}$ consisting of the "diagonal" elements $\{x, x\}$ of $E^{\prime}$. Further, from the theorem of $\$ 5$ we obtain the class of functions and the norm corresponding to the kernel $K$. Thus we have the following theorem.

THEOREM II. The kernel $K(x, y)=K_{1}(x, y) K_{2}(x, y)$ is the reproducing kernel of the class $F$ of the restrictions of all functions of the direct product $F^{\prime}=F_{1} \otimes F_{2}$ to the diagonal set $E_{1}$ formed by all the elements $\{x, x\} \in E^{\prime}$. For any such restriction $f,\|f\|=\min \left\|g^{\prime}\right\|^{\prime}$ for all $g^{\prime} \in F^{\prime}$, the restriction of which to the diagonal set $E_{1}$ is $f$.

REMARK. Let $\left\{g_{1}^{(k)}\right\}$ be a complete orthonormal system in $F_{1}$. Then every function $f \in F$ is representable as a series

$$
f(x)=\sum_{1}^{\infty} f_{2}^{(k)}(x) g_{1}^{(k)}(x), f_{2}^{(k)} \in F_{2}, \sum_{1}^{\infty}\left\|f_{2}^{(k)}\right\|_{2}^{2}<\infty .
$$

Among all such representations of $f(x)$ there exists one (and only one) 
which gives its minimum to the sum $\sum\left\|f_{2}^{(k)}\right\|_{2}^{2}$. This minimum is equal to $\|f\|^{2}$.

We can apply Theorem II to a class $F$ and its conjugate $\bar{F}$. The product of the corresponding kernels is $|K(x, y)|^{2}=K(x, y) K(y, x)$ and the corresponding class may be obtained from the remark above.

9. Limits of reproducing kernels. We shall consider two cases: (A) essentially, the case of a decreasing sequence of classes $F_{1} \supset F_{2} \supset \cdots$ with a decreasing sequence of kernels $K_{1} \gg K_{2} \gg K_{3} \gg \cdots$; (B) essentially, the case of an increasing sequence of classes and kernels.

A. The case of a decreasing sequence. Let $\left\{E_{n}\right\}$ be an increasing sequence of sets, $E$ their sum

$$
E=E_{1}+E_{2}+\cdots, E_{1} \subset E_{2} \subset \cdots .
$$

Let $F_{n}, n=1,2, \cdots$, be a class of functions defined in $E_{n}$. For a function $f_{n} \in F_{n}$ we shall denote by $f_{n m}, m \leqq n$, the restriction of $f_{n}$ to the set $E_{m} \subset E_{n}$ $\left(f_{n n}=f_{n}\right)$. We shall suppose then that the classes $F_{n}$ form a decreasing sequence in the sense

$$
\text { for every } f_{n} \in F_{n} \text { and every } m \leqq n, \quad f_{n m} \in F_{m} \text {. }
$$

Suppose further that the norms \|\|$_{n}$ defined in $F_{n}$ form an increasing sequence in the sense

$$
\text { for every } f_{n} \in F_{n} \text { and every } m \leqq n, \quad\left\|f_{n m}\right\|_{m} \leqq\left\|f_{n}\right\|_{n} .
$$

Finally, we suppose that every $F_{n}$ possesses a reproducing kernel $K_{n}(x, y)$.

The case of all sets $E_{n}$ equal, $E_{1}=E_{2}=\cdots=E$, is not excluded. Clearly, in this case $f_{n m}=f_{n}, F_{n} \subset F_{m}$, and, following Theorem II of $\S 7$, it is enough to suppose the existence of $K_{1}(x, y)$ in order to deduce the existence of all $K_{n}$ and to obtain the property $K_{n} \ll K_{m}$ for $m<n$.

In the general case we have to introduce the restrictions $K_{n m}$ of $K_{n}$ to the set $E_{m}(m \leqq n)$. By the theorem of $\S 5, K_{n m}$ is the r.k. of the class $F_{n m}$ of all restrictions $f_{n m}$ for $f_{n} \in F_{n}$. The norm in $F_{n m}$ is given by

$$
\left\|f_{n m}\right\|_{n m}=\min \left\|f_{n}^{\prime}\right\|_{n} \quad \text { for all } f_{n}^{\prime} \text { with } f_{n m}^{\prime}=f_{n m} .
$$

From (3) we get

$$
\left\|f_{n m}\right\|_{n m} \geqq\left\|f_{n m}\right\|_{m}
$$

and consequently, by Theorem II of $\S 7$,

$$
K_{n m} \ll K_{m}, \quad m<n .
$$

We shall now prove the following theorem.

THEOREM I. Under the above assumptions on the classes $F_{n}$, the kernels $K_{n}$ converge to a kernel $K_{0}(x, y)$ defined for all $x, y$ in $E . K_{0}$ is the r.k. of the class $F_{0}$ of all functions $f_{0}$ defined in $E$ such that $1^{\circ}$ their restrictions $f_{0 n}$ in $E_{n}$ 
belong to $F_{n}, n=1,2, \cdots, 2^{\circ} \lim _{n=\infty}\left\|f_{0 n}\right\|_{n}<\infty$. The norm of $f_{0} \in F_{0}$ is given by $\left\|f_{0}\right\|_{0}=\lim _{n=\infty}\left\|f_{0 n}\right\|_{n}$.

REMARK. Condition $1^{\circ}$ implies, following (3), that $\lim _{n=\infty}\left\|f_{0 n}\right\|_{n}$ exists, but it may be infinite.

Proof. The convergence of $K_{n}$ to $K$ is to be understood in this way: any two points $x, y$ in $E$ belong to all $E_{n}$ starting from some $E_{n_{0}}$ on. Consequently $K_{n}(x, y)$ are defined for $n>n_{0}$ and we have to prove $\lim _{n=\infty} K_{n}(x, y)=K(x, y)$.

For fixed $y \in E_{k}$ and $k \leqq m \leqq n$ we have, following our assumptions,

$$
\begin{aligned}
&\left\|K_{m k}(x, y)-K_{n k}(x, y)\right\|_{k}^{2} \leqq\left\|K_{m}(x, y)-K_{n m}(x, y)\right\|_{m}^{2} \\
&=K_{m}(y, y)-\overline{K_{n m}(y, y)}-K_{n m}(y, y)+\left\|K_{n m}(x, y)\right\|_{m}^{2} \\
& \leqq K_{m}(y, y)-2 K_{n}(y, y)+\left\|K_{n}(x, y)\right\|_{n}^{2} \\
&=K_{m}(y, y)-K_{n}(y, y) .
\end{aligned}
$$

From (4) it follows that $K_{m}-K_{n m}$ is a p.d. matrix. Therefore $K_{m}(y, y)$ $-K_{n m}(y, y)=K_{m}(y, y)-K_{n}(y, y) \geqq 0$, and the sequence $\left\{K_{m}(y, y)\right\}_{m \geqq k}$ is a decreasing sequence of non-negative numbers. Consequently it is a convergent sequence. (5) shows then that the functions $K_{m k}(x, y) \in F_{k}$, for fixed $k$ and $m \rightarrow \infty$, converge strongly in $F_{k}$ to some function $\phi_{k}(x) \in F_{k}$. This involves $\lim _{m=\infty} K_{m k}(x, y)=\lim _{m=\infty} K_{m}(x, y)=\phi_{k}(x)$ for every $x \in E_{k}$.

Since for every $x, y$ in $E$ we can choose a $k$ so that $x$ and $y$ belong to $E_{k}$, it is clear that $K_{m}(x, y)$ converge and that the limit $K_{0}(x, y)$ does not depend upon the choice of $k$. The function $\phi_{k}(x)$ is clearly the restriction $K_{0 k}(x, y)$ of $K_{0}$ to $E_{k}$. Consequently $K_{m k}(x, y)$, for fixed $y$, converges strongly in $F_{k}$ to $K_{0 k}(x, y)$ which belongs to $F_{k}$. We then obtain from (5), by taking $n \rightarrow \infty$,

(6) $\left\|K_{m k}(x, y)-K_{0 k}(x, y)\right\|_{k}^{2} \leqq K_{m}(y, y)-K_{0}(y, y)$;

$$
\begin{aligned}
\left\|K_{0 k}(x, y)\right\|_{k} & \leqq\left\|K_{0 k}(x, y)-K_{m k}(x, y)\right\|_{k}+\left\|K_{m k}(x, y)\right\|_{k} \\
& \leqq\left(K_{m}(y, y)-K_{0}(y, y)\right)^{1 / 2}+\left\|K_{m}(x, y)\right\|_{m} \\
& \leqq\left(K_{m}(y, y)-K_{0}(y, y)\right)^{1 / 2}+\left(K_{m}(y, y)\right)^{1 / 2}
\end{aligned}
$$

and for $m \rightarrow \infty,\left\|K_{0 k}(x, y)\right\|_{k}^{2} \leqq K_{0}(y, y)$.

Therefore for each $y \in E, K_{0}(x, y)$, as function of $x$, belongs to the class $F_{0}$ of our theorem.

Let us now prove that the class $F_{0}$ is a Hilbert space.

$F_{0}$ is linear, since $\left\|\alpha f_{0 n}+\beta g_{0 n}\right\|_{n} \leqq|\alpha|\left\|f_{0 n}\right\|_{n}+|\beta|\left\|g_{0 n}\right\|_{n}$.

$\left\|f_{0}\right\|_{0}^{2}$ is a quadratic form, since

$$
\begin{aligned}
\left\|\alpha f_{0}+\beta g_{0}\right\|_{0}^{2} & =\lim _{n=\infty}\left\|\alpha f_{0 n}+\beta g_{0 n}\right\|_{n}^{2} \\
& =\lim _{n=\infty}\left[\alpha \bar{\alpha}\left\|f_{0 n}\right\|_{n}^{2}+\alpha \bar{\beta}\left(f_{0 n}, g_{0 n}\right)_{n}+\bar{\alpha} \beta\left(g_{0 n}, f_{0 n}\right)_{n}+\beta \bar{\beta}\left\|g_{0 n}\right\|_{n}^{2}\right] .
\end{aligned}
$$


Since the quadratic form in square brackets converges for all values of the complex variables $\alpha, \beta$, it converges to a form of the same kind. This proves that $\left\|f_{0}\right\|_{0}^{2}$ is a quadratic form and also that

$$
\left(f_{0}, g_{0}\right)_{0}=\lim _{n=\infty}\left(f_{0 n}, g_{0 n}\right)_{n} \text {. }
$$

It remains to be proved that $F_{0}$ is complete.

Take a Cauchy sequence $\left\{f_{0}^{(n)}\right\} \subset F_{0}$. The inequality $\left\|f_{0 k}^{(m)}-f_{0 k}^{(n)}\right\|_{k}$ $\leqq\left\|f_{01}^{(m)}-f_{01}^{(n)}\right\|_{1}$, for $l>k$, gives, when $l \rightarrow \infty,\left\|f_{0 k}^{(m)}-f_{0 k}^{(n)}\right\|_{k} \leqq\left\|f_{0}^{(m)}-f_{0}^{(n)}\right\|_{0}$. Hence, $\left\{f_{0 k}^{(m)}\right\}_{m=1,2}, \ldots$ is a Cauchy sequence in $F_{k}, \lim _{m=\infty} f_{0 k}^{(m)}=\psi_{k} \in F_{k}$. It is clear that $\psi_{k}$ is the restriction to $E_{k}$ of a function $\psi_{0}$ defined in $E$. We have

$$
\left\|f_{0 k}^{(m)}-\psi_{0 k}\right\|_{k}=\lim _{n=\infty}\left\|f_{0 k}^{(m)}-f_{0 k}^{(n)}\right\|_{k} \leqq \lim _{n=\infty}\left\|f_{0}^{(m)}-f_{0}^{(n)}\right\|_{0} .
$$

This allows us to prove that $\psi_{0} \in F_{0}$, since it gives a bound for $\left\|\psi_{0 k}\right\|_{k}$ independent of $k$, namely

$$
\left\|\psi_{0 k}\right\|_{k} \leqq\left\|f_{0 k}^{(m)}\right\|_{k}+\lim _{n=\infty}\left\|f_{0}^{(m)}-f_{0}^{(n)}\right\|_{0} \leqq\left\|f_{0}^{(m)}\right\|_{0}+\lim _{n=\infty}\left\|f_{0}^{(m)}-f_{0}^{(n)}\right\|_{0}
$$

On the other hand, it shows that

$$
\left\|f_{0}^{(m)}-\psi_{0}\right\|_{0}=\lim _{k=\infty}\left\|f_{0 k}^{(m)}-\psi_{0 k}\right\|_{k} \leqq \lim _{n=\infty}\left\|f_{0}^{(m)}-f_{0}^{(n)}\right\|_{0}
$$

and consequently $\lim _{m=\infty}\left\|f_{0}^{(m)}-\psi_{0}\right\|_{0}=0$. This achieves the proof of completeness.

We have still to prove the reproducing property of $K_{0}$. To this effect take any $f_{0} \in F_{0}, y \in E$. For sufficiently large $n$ we have

$$
\begin{aligned}
f_{0}(y)=f_{0 n}(y)=\left(f_{0 n}(x), K_{n}(x, y)\right)_{n}=\left(f_{0 n}(x)\right. & \left., K_{0 n}(x, y)\right)_{n} \\
& +\left(f_{0 n}(x), K_{n}(x, y)-K_{0 n}(x, y)\right)_{n} .
\end{aligned}
$$

For $n \rightarrow \infty$, the first scalar product in the last member converges, by formula (7), to $\left(f_{0}, K_{0}(x, y)\right)_{0}$. The second scalar product converges to 0 ; in fact, by formula (6) (with $k=m=n$ ), it is in absolute value smaller than

$$
\left\|f_{0 n}\right\|_{n} K_{n}(x, y)-K_{0 n}(x, y)\left\|_{n} \leqq\right\| f_{0} \|_{0}\left(K_{n}(y, y)-K_{0}(y, y)\right)^{1 / 2} \text {. }
$$

This achieves the proof of our theorem.

B. The case of an increasing sequence. Let $\left\{E_{n}\right\}$ be a decreasing sequence of sets, $E$ their intersection

$$
E=E_{1} \cdot E_{2} \cdots, E_{1} \supset E_{2} \supset \cdots \text {. }
$$

Let $F_{n}$ be a class of functions defined in $E_{n}$. As before, we define the restriction $f_{n m}$, for $f_{n} \in F_{n}$, but now $m$ has to be greater than $n$. We suppose then that $F_{n}$ form an increasing sequence 


$$
\text { for every } f_{n} \in F_{n} \text { and every } m \geqq n, \quad f_{n m} \in F_{m} \text {. }
$$

We suppose further that the norms \|\|$_{n}$ form a decreasing sequence

$$
\text { for every } f_{n} \in F_{n} \text { and every } m \geqq n, \quad\left\|f_{n m}\right\|_{m} \leqq\left\|f_{n}\right\|_{n} \text {. }
$$

Finally, we suppose that every $F_{n}$ possesses a r.k. $K_{n}(x, y)$.

Now, even for all $E_{n}$ equal, we cannot deduce the existence of all kernels $K_{n}$ from the existence of one of them.

As in the case $\mathrm{A}$, we get for the restrictions $K_{n m}$ of $K_{n}$ the formula

$$
K_{n m} \ll K_{m},
$$

for $m>n$.

For $y \in E,\left\{K_{m}(y, y)\right\}$ is an increasing sequence of positive numbers. Its limit may be infinite. We define, consequently,

$$
E_{0}=\text { set of } y \in E \text {, such that } K_{0}(y, y)=\lim _{m=\infty} K_{m}(y, y)<\infty .
$$

For an illustration of this point consider Bergman's kernels $K_{n}$ for a decreasing sequence of domains $E_{n}$. If the intersection $E$ of the domains $E_{n}$ is composed of a closed circle with an exterior segment attached to it, the set $E_{0}$ will be composed of all interior points of the circle.

We suppose that $E_{0}$ is not empty and define the limit-class of the classes $F_{n}$ in the following way: let $F_{0}$ be the class of all restrictions $f_{n 0}$ of functions $f_{n} \in F_{n}(n=1,2, \cdots)$ to the set $E_{0}$. From (10), we know that the sequence $\left\{\left\|f_{n k}\right\|_{k}\right\}_{k=n, n+1}, \cdots$ is decreasing and we can define

$$
\left\|f_{n 0}\right\|_{0}=\lim _{k=\infty}\left\|f_{n k}\right\|_{k} \text {. }
$$

As in case $\mathrm{A}$ we prove that $\left\|f_{n 0}\right\|_{0}^{2}$ is a positive quadratic form. This form is positive definite since from $\left\|f_{n 0}\right\|_{0}=0$ it follows that for any $y \in E_{0},\left|f_{n 0}(y)\right|$ $=\left|f_{n k}(y)\right|=\left|\left(f_{n k}(x), K_{k}(x, y)\right)_{k}\right| \leqq\left\|f_{n k}\right\|_{k}\left(K_{k}(y, y)\right)^{1 / 2} \rightarrow\left\|f_{n 0}\right\|_{0}\left(K_{0}(y, y)\right)^{1 / 2}=0$, that is, $f_{n 0}=0$. Consequently \|\|$_{0}$ is a norm in $F_{0}$.

In general $F_{0}$ will not be complete. In order that $F_{0}$ admit of a functional completion with a reproducing kernel, there are two conditions to be fulfilled which are given in the theorem of $\$ 4$. The first one is that for every $y$ there exists a constant $M_{y}$ so that

$$
\left|f_{n 0}(y)\right| \leqq M_{y}\left\|f_{n 0}\right\|_{0} \quad \text { for all } f_{n 0} \in F_{0} .
$$

Let us remark that the functions $f_{n 0}$ may be considered as defined in the whole set $E$ (taking the restriction of $f_{n}$ to $E$ ). Then, for every $y \in E$, the condition (14) is equivalent to $K_{0}(y, y)=\lim K_{n}(y, y)<\infty$. In fact, from the latter condition it follows in the same manner as above that $\left|f_{n 0}(y)\right|$ $\leqq\left\|f_{n 0}\right\|_{0}\left(K_{0}(y, y)\right)^{1 / 2}$, that is, (14) with $M_{y}=\left(K_{0}(y, y)\right)^{1 / 2}$. From (14), by taking $f_{n}(x)=K_{n}(x, y)$ we get $K_{n}(y, y) \leqq M_{y}\left\|K_{n 0}(x, y)\right\|_{0} \leqq M_{y}\left\|K_{n}(x, y)\right\|_{n}$ $=M_{y}\left(K_{n}(y, y)\right)^{1 / 2}$, that is, $K_{n}(y, y) \leqq M_{y}^{2}$. 
Consequently, condition (14), that is, condition $1^{\circ}$ of $\$ 4$, is assured by our restriction to the set $E_{0}$. But the condition $2^{\circ}$ of $\$ 4$ is in general not assured.

We may illustrate this by the counter-example of $\$ 4$. Take all the $E_{n}$ equal to the set $E$ of this counter-example. As class $F_{n}$ we take the $n$-dimensional subspace of the class $F$ introduced there, consisting of all polynomials of degree $\leqq n$. Each $F_{n}$ is a complete space and its r.k. $K_{n}$ converges to the Bergman kernel of the circle, restricted to $E$. Consequently $E_{0}=E, F_{0}=F$ and \|\|$_{0}$ is clearly the norm introduced there.

To overcome this difficulty we can proceed as indicated in $\$ 4$. We complete $F_{0}$ by ideal elements; in the completed Hilbert space $\bar{F}_{0}$ we choose an additional set $E^{\prime}$ of ideal elements such that the functions of $F_{0}$, extended to $E_{0}+E^{\prime}$, with the same norm as in $F_{0}$, form a space admitting a functional completion leading to a class $\widetilde{F}_{0}$ with a reproducing kernel $\widetilde{K}_{0}$.

Following the theorem of $\S 5$, we can return now to our set $E_{0}$ by restricting the functions of $\tilde{F}_{0}$ to $E_{0}$. If we take in the restricted class the norm defined in the theorem of $\S 5$, we shall get as r.k. the restriction of $\tilde{K}_{0}$ to $E_{0}$. The restricted class $F_{0}^{*}$ and its norm \|\|$_{0}^{*}$ can then be described, in terms of the space $F_{0}$ and its norm \|\|$_{0}$, in the following way.

$f_{0}^{*} \in F_{0}^{*}$ if there is a Cauchy sequence $\left\{f_{0}^{(n)}\right\} \subset F_{0}$ such that

$$
\begin{aligned}
f_{0}^{*}(x) & =\lim _{n=\infty} f_{0}^{(n)}(x) \quad \text { for every } x \in E_{0}, \\
\left\|f_{0}^{*}(x)\right\|_{0}^{*} & =\min \lim _{n=\infty}\left\|f_{0}^{(n)}\right\|_{0},
\end{aligned}
$$

the minimum being taken for all Cauchy sequences $\left\{f_{0}^{(n)}\right\} \subset F_{0}$ satisfying (15). There exists at least one Cauchy sequence for which the minimum is attained. Such sequences will be called determining $f_{0}^{*}$.

The scalar product corresponding to \|\|$_{0}^{*}$ is defined by

$$
\left(f_{0}^{*}, g_{0}^{*}\right)_{0}^{*}=\lim _{n=\infty}\left(f_{0}^{(n)}, g_{0}^{(n)}\right)_{0}
$$

for any two Cauchy sequences $\left\{f_{0}^{(n)}\right\}$ and $\left\{g_{0}^{(n)}\right\}$ determining $f_{0}^{*}$ and $g_{0}^{*}$.

It is important to note that formula (17) is still valid when only one of the sequences $\left\{f_{0}^{(n)}\right\},\left\{g_{0}^{(n)}\right\}$ is determining, the other satisfying only (15).

All these facts about the space $F_{0}^{*}$ and its norm and scalar product are easily obtained when we form, as in $\S 5$, the subspace $\widetilde{F}_{0}^{0} \subset \widetilde{F}_{0}$ of all $\tilde{f}_{0} \in \widetilde{F}_{0}$ vanishing in $E_{0}$. The complementary subspace $\widetilde{F}_{0}^{\prime}=\widetilde{F}_{0} \ominus \widetilde{F}_{0}^{0}$ is then in an isomorphic correspondence with $F_{0}^{*}, \tilde{f}_{0}^{\prime} \rightarrow f_{0}^{*}$, where $f_{0}^{*}$ is the restriction of $\tilde{f}_{0}^{\prime}$ to $E_{0}$. Further,

$$
\left\|f_{0}^{*}\right\|_{0}^{*}=\left\|\tilde{f}_{0}^{\prime}\right\|_{0}, \quad\left(f_{0}^{*}, g_{0}^{*}\right)_{0}^{*}=\left(\tilde{f}_{0}^{\prime}, \tilde{g}_{0}^{\prime}\right)_{0}
$$


where \|\|$_{0}$ and $(,)_{0}$ are the norm and scalar product in $\widetilde{F}_{0}$.

A Cauchy sequence $\left\{f_{0}^{n}\right\} \subset F_{0}$ converges in $\tilde{F}_{0}$ to a function $\tilde{f}_{0}$. In the set $E_{0}$ it converges everywhere to a function $f_{0}^{*} \in F_{0}^{*}$ which is the restriction of $\tilde{f}_{0}$ to $E_{0} . f_{0}^{*}$ is also the restriction of $\tilde{f}_{0}^{\prime}=$ projection of $\tilde{f}_{0}$ on $\tilde{F}_{0}^{\prime}$. Since $\tilde{f}_{0}-\tilde{f}_{0}^{\prime}$ $\in \tilde{F}_{0}^{0}$, we get

$$
\lim _{n=\infty}\left\|f_{0}^{(n)}\right\|_{0}^{2}=\left\|\tilde{f}_{0}\right\|_{0}^{2}=\left\|\tilde{f}_{0}^{\prime}\right\|_{0}^{2}+\left\|\tilde{f}_{0}-\tilde{f}_{0}^{\prime}\right\|_{0}^{2} \geqq\left\|f_{0}^{*}\right\|_{0}^{* 2} .
$$

The equality here is attained if $\left\{f_{0}^{(n)}\right\}$ converges in $\widetilde{F}_{0}$ to $\tilde{f}_{0}^{\prime}$. Such a Cauchy sequence we have called as determining $f_{0}^{*}$. If now two Cauchy sequences $\left\{f_{0}^{(n)}\right\}$ and $\left\{g_{0}^{(n)}\right\}$ converge everywhere in $E_{0}$ to $f_{0}^{*}$ and $g_{0}^{*}$, they converge in $\widetilde{F}_{0}$ to vectors $\tilde{f}_{0}$ and $\tilde{g}_{0}$ whose restrictions to $E_{0}$ are $f_{0}^{*}$ and $g_{0}^{*}$. If $\bar{f}_{0}^{\prime}$ and $\tilde{g}_{0}^{\prime}$ are projections of $\tilde{f}_{0}$ and $\tilde{g}_{0}$ on $\tilde{F}_{0}^{\prime}$, then $\left(f_{0}^{*}, g_{0}^{*}\right)_{0}^{*}=\left(\tilde{f}_{0}^{\prime}, \tilde{g}_{0}^{\prime}\right)_{0}^{\tilde{D}}$, but $\lim \left(f_{0}^{(n)}, g_{0}^{(n)}\right)_{0}$ $=\left(\tilde{f}_{0}, \tilde{g}_{0}\right)_{0}$. If one of the sequences, say $\left\{f_{0}^{(n)}\right\}$, determines its limit in $E_{0}$, then $\tilde{f}_{0}=\tilde{f}_{0}^{\prime}$, and $\left(f_{0}^{*}, g_{0}^{*}\right)_{0}^{*}=\left(\tilde{f}_{0}^{\prime}, \tilde{g}_{0}^{\prime}\right)_{0}^{\tilde{0}}=\left(\tilde{f}_{0}^{\prime}, \tilde{g}_{0}\right)_{0} \tilde{=}=\lim \left(f_{0}^{(n)}, g_{0}^{(n)}\right)_{0}$.

The space $F_{0}^{*}$ being completely defined we prove the following theorem.

THEOREM II. The restrictions $K_{n 0}(x, y)$ for every fixed $y \in E_{0}$ form a Cauchy sequence in $F_{0}$. They converge to a function $K_{0}^{*}(x, y) \in F_{0}^{*}$ which is the reproducing kernel of $F_{0}^{*}$.

Proof. By an argument similar to the one used in (5) we obtain for $n \leqq m \leqq k$

$$
\left\|K_{m k}(x, y)-K_{n k}(x, y)\right\|_{k}^{2} \leqq K_{m}(y, y)-K_{n}(y, y) .
$$

Taking $k \rightarrow \infty$, we have

$$
\left\|K_{m 0}(x, y)-K_{n 0}(x, y)\right\|_{0}^{2} \leqq K_{m}(y, y)-K_{n}(y, y) .
$$

This proves, together with (12), that $\left\{K_{n 0}(x, y)\right\}$ is a Cauchy sequence in $F_{0}$. By property (14) this sequence converges for every $x \in E_{0}$ to a function $K_{0}^{*}(x, y)$ which, by definition (15), belongs to $F_{0}^{*}$.

It remains to prove the reproducing property of $K_{0}^{*}$. To this effect take any $f_{0}^{*} \in F_{0}^{*}$ and a Cauchy sequence $\left\{f_{0}^{(n)}\right\} \subset F_{0}$ determining $f_{0}^{*}$. Each $f_{0}^{(n)}$ is a restriction of some $f_{k_{n}} \in F_{k_{n}}, f_{0}^{(n)}=f_{k_{n} 0}$. By (13) there exists an increasing sequence $m_{1}<m_{2}<\cdots$ such that

$$
m_{n}>k_{n}, \quad\left\|f_{k_{n} m_{n}}\right\|_{m_{n}}^{2}-\left\|f_{k_{n}}\right\|_{0}^{2} \leqq \frac{1}{n^{2}} .
$$

It is clear that $\left\{K_{m_{n} 0}(x, y)\right\}$ is also a Cauchy sequence converging to $K_{0}^{*}(x, y)$. Consequently, from (17) it follows that

$$
\left(f_{0}^{*}(x), K_{0}^{*}(x, y)\right)_{0}^{*}=\lim _{n=\infty}\left(f_{k_{n} 0}(x), K_{m_{n} 0}(x, y)\right)_{0} .
$$

We may now write 


$$
\begin{aligned}
\left(f_{k_{n} 0}(x), K_{m_{n} 0}(x, y)\right)_{0} & =\left(f_{k_{n} m_{n}}(x), K_{m_{n}}(x, y)\right)_{m_{n}} \\
& -\left[\left(f_{k_{n} m_{n}}(x), K_{m_{n}}(x, y)\right)_{m_{n}}-\left(f_{k_{n} 0}(x), K_{m_{n} 0}(x, y)\right)_{0}\right] .
\end{aligned}
$$

The square bracket is of the form $\left[(g, h)_{m_{n}}-\left(g_{0}, h_{0}\right)_{0}\right]$ for $g, h$ of $F_{m_{n}}\left(g_{0}, h_{0}\right.$ are restrictions of $g, h$ to $E_{0}$ ). This is a bilinear form in $g, h$ and the corresponding quadratic form $(g, g)_{m_{n}}-\left(g_{0}, g_{0}\right)_{0}=\|g\|_{m_{n}}^{2}-\left\|g_{0}\right\|_{0}^{2}$ is positive (following (10) and (13)). Consequently the Cauchy-Schwarz inequality is valid for this form and in the case of the square bracket of (22) it gives in connection with (20)

$$
\begin{aligned}
|[\cdots]| & \leqq\left[\left\|f_{k_{n} m_{n}}\right\|_{m_{n}}^{2}-\left\|f_{k_{n} 0}\right\|_{0}^{2}\right]^{1 / 2}\left[\left\|K_{m_{n}}(x, y)\right\|_{m_{n}}^{2}-\left\|K_{m_{n} 0}(x, y)\right\|_{0}^{2}\right]^{1 / 2} \\
& \leqq \frac{1}{n}\left\|K_{m_{n}}(x, y)\right\|_{m_{n}}=\frac{1}{n} K_{m_{n}}(y, y)^{1 / 2} .
\end{aligned}
$$

For $n \rightarrow \infty$ this converges to 0 , since $K_{m_{n}}(y, y) \nearrow K_{0}(y, y)<\infty$. Therefore, (21) and (22) yield

$$
\begin{aligned}
\left(f_{0}^{*}(x), K_{0}^{*}(x, y)\right)_{0}^{*} & =\lim _{n=\infty}\left(f_{k_{n} m_{n}}(x), K_{m_{n}}(x, y)\right)_{m_{n}}=\lim _{n=\infty} f_{k_{n} m_{n}}(y) \\
& =\lim f_{k_{n} 0}(y)=\lim f_{0}^{(n)}(y)=f_{0}^{*}(y),
\end{aligned}
$$

which is the reproducing property of $K_{0}^{*}$.

REMARK. A particularly simple case is the one where the class $F_{0}$ with the norm \|\|$_{0}$ happens to be a subspace of a class $F$ possessing a reproducing kernel. Then, condition $2^{\circ}$ of $\$ 4$ is clearly satisfied; $F_{0}^{*}$ is the functional completion of $F_{0}$, the norm \|\|$_{0}^{*}$ is an extension of the norm \|\|$_{0}$, and $F_{0}^{*}$ is simply the closure in $F$ of $F_{0}$.

A trivial case of this kind is one where the $F_{n}$ form an increasing sequence of subspaces of a class $F$ with a r.k. Hence $E_{1}=E_{2}=\cdots=E=E_{0}$ and $F_{0}^{*}$ is the closure of the sum $\sum F_{n}$.

10. Construction of a r.k. by resolution of identity. Let us give a brief résumé of the essential properties of resolutions of identity in a Hilbert space $\mathfrak{S}$ (for a complete study of resolutions of identity, especially in connection with the theory of operators, see M. H. Stone [1]). For simplicity's sake we shall suppose here that the space $\mathfrak{S}$ is separable.

We call a resolution of identity a class $\left\{P_{\lambda}\right\}$ of projections in $\mathfrak{H}$, depending on a real parameter $\lambda,-\infty<\lambda<+\infty$, and having the following properties:

1. $P_{\lambda}$ is a projection on a closed subspace $\mathfrak{S}_{\lambda} \subset \mathfrak{S}_{\text {, }}$, increasing with $\lambda: \mathfrak{S}_{\lambda^{\prime}} \subset \mathfrak{S}_{\lambda}$ for $\lambda^{\prime}<\lambda$.

2. $P_{\lambda} \rightarrow 0$ for $\lambda \rightarrow-\infty ; P_{\lambda} \rightarrow I$ (identity operator) for $\lambda \rightarrow+\infty$.

For any open interval $\Delta: \lambda^{\prime}<\lambda<\lambda^{\prime \prime}$, we define

$$
\Delta \mathfrak{S}=\mathfrak{S}_{\lambda^{\prime \prime}} \ominus \mathfrak{S}_{\lambda^{\prime}}, \quad \Delta P=P_{\lambda^{\prime \prime}}-P_{\lambda^{\prime}} .
$$


$\Delta P$ is the projection on $\Delta \mathfrak{S}$.

For any decomposition of the real axis into intervals $\Delta_{k}=\left(\lambda_{k}, \lambda_{k+1}\right)$, $-\infty \leftarrow \cdots<\lambda_{-2}<\lambda_{-1}<\lambda_{0}<\lambda_{1}<\lambda_{2}<\cdots \rightarrow+\infty$, we have, obviously,

$$
I=\sum_{k} \Delta_{k} P
$$

the series converging in the sense of strong limit for operators.

A real number $\lambda_{0}$ belongs to the spectrum of $\left\{P_{\lambda}\right\}$ if $\Delta P \neq 0$ for every interval $\Delta$ containing $\lambda_{0}$. The numbers belonging to the spectrum form a closed set.

For any real $\theta$ and any decreasing sequence of intervals $\Delta_{n}$ containing $\theta$ and converging to $\theta$ there exist the limits

$$
\delta_{\theta} P=\lim \Delta_{n} P, \quad \delta_{\theta} \mathfrak{S}=\lim \Delta_{n} \mathfrak{S},
$$

the second limit being the intersection of the decreasing sequence of subspaces $\Delta_{n} \mathfrak{S}$. These limits do not depend on the choice of $\Delta_{n}$ and $\delta_{\theta} P$ is the projection on $\delta_{\theta} \mathfrak{S}$. Only for an enumerable set of $\theta$, say $\left\{\theta_{k}\right\}$, is $\delta_{\theta} P \neq 0$.

If we have $\delta_{\theta_{n}} P=I$, which means $\delta_{\theta_{1}} \mathfrak{S} \dot{+} \delta_{\theta_{2}} \mathfrak{S} \dot{+} \cdots=\mathfrak{S}$, we say that the spectrum of $\left\{P_{\lambda}\right\}$ is discrete.

If, for all $\theta, \delta_{\theta} P=0$, we say that the spectrum of $\{P\}$ is continuous (often called purely continuous).

In our applications we shall meet, essentially, only discrete spectra or continuous spectra.

It has been proved (theorem of Hellinger-Hahn) that for any spectrum there exist finite or infinite systems $\left\{f_{n}(\lambda)\right\}$ of elements $f_{n}(\lambda) \in \mathcal{S}$, depending on $\lambda$, such that if we denote by $\Delta f_{n}$ the difference $f_{n}\left(\lambda^{\prime \prime}\right)-f_{n}\left(\lambda^{\prime}\right)$, we have

(a) for $m \neq n,\left(\Delta_{1} f_{m}, \Delta_{2} f_{n}\right)=0$ for any intervals $\Delta_{1}, \Delta_{2}$.

(b) $\left(\Delta_{1} f_{n}, \Delta_{2} f_{n}\right)=0$ for any non-overlapping intervals $\Delta_{1}, \Delta_{2}$.

(c) For every interval $\Delta$, the elements $\Delta_{1} f_{n}, n=1,2, \cdots$ for all $\Delta_{1} \subset \Delta$, belong to the subspace $\Delta \mathfrak{S}$ and form a complete system in $\Delta \mathfrak{S}$.

The minimal number of elements in such a system $\left\{f_{n}(\lambda)\right\}$ is called the multiplicity of the spectrum. The spectrum is called simple if the multiplicity $=1$, that is, if there exists such a system with only one element $f_{1}(\lambda)$.

In the case of a discrete spectrum the multiplicity is the maximal dimension of the subspaces $\delta_{\theta} \mathfrak{S}$.

If a system of elements $\left\{f_{n}(\lambda)\right\}$ satisfies (a) and (b) and instead of (c) satisfies the weaker condition

( $\left.c^{\prime}\right)$ The elements $\Delta f_{n}$ for $n=1,2, \cdots$ and for all intervals $\Delta$ form a complete system in $\mathfrak{S}$,

then the system $\left\{f_{n}(\lambda)\right\}$ determines a corresponding resolution of identity $\left\{P_{\lambda}\right\}$ (for which it satisfies (c)) in the following manner:

$\mathfrak{S}_{\lambda}$ is the subspace generated by all the $\Delta f_{n}, n=1,2, \cdots, \Delta=\left(\lambda^{\prime}, \lambda^{\prime \prime}\right)$ with $\lambda^{\prime \prime}<\lambda$. 
This resolution of identity is continuous to the left, that is, $\mathfrak{S}_{\lambda}=\lim \mathfrak{S}_{\lambda^{\prime}}$ for $\lambda^{\prime} \lambda \lambda$. This condition (or the right side continuity) is usually accepted, for reasons of convenience, as an additional condition on resolutions of identity.

For any system $\left\{f_{n}(\lambda)\right\}$ satisfying (a), (b), and (c), it is seen that $\left\|f_{n}(\lambda)\right\|^{2}$ is a non-decreasing function $\mu_{n}(\lambda), \Delta \mu_{n}=\mu_{n}\left(\lambda^{\prime \prime}\right)-\mu_{n}\left(\lambda^{\prime}\right)=\left\|\Delta f_{n}\right\|^{2}$. We consider the measure $\mu_{n}$, introduced on the real axis by $\mu_{n}(\lambda)$, which leads to the Lebesgue-Stieltjes integral $\int \Phi(\lambda) d \mu_{n}(\lambda)$. It has been proved that for every $u \in \mathfrak{S}$ there exists the limit

$$
\phi_{n}(\lambda)=\lim _{\lambda^{\prime} / \lambda, \lambda^{\prime \prime} \backslash \lambda} \frac{\left(u, \Delta f_{n}\right)}{\left\|\Delta f_{n}\right\|^{2}}=\frac{d\left(u, f_{n}(\lambda)\right)}{d \mu_{n}(\lambda)},
$$

for all $\lambda$ with exception of a set of $\mu_{n}$-measure 0 .

We have further

$$
\begin{gathered}
\|u\|^{2}=\sum_{n} \int_{-\infty}^{\infty}\left|\phi_{n}(\lambda)\right|^{2} d \mu_{n} . \\
(u, v)=\sum_{n} \int_{-\infty}^{\infty} \phi_{n}(\lambda) \overline{\psi_{n}(\lambda)} d \mu_{n}, \text { where } \psi_{n}(\lambda)=\frac{d\left(v, f_{n}(\lambda)\right)}{d \mu_{n}(\lambda)} .
\end{gathered}
$$

Let us now apply the above considerations to the construction of a r.k. We suppose that our Hilbert space $\mathfrak{E}$ is a class of functions defined in $E$ with a r.k. $K(x, y)$.

For a given resolution of identity $\left\{P_{\lambda}\right\}$ every subspace $\Delta \mathfrak{W}$ will have a r.k. which we shall denote by $\Delta K(x, y)$. The kernel $\Delta K$ determines the projection $\Delta P$ by the equation

$$
\Delta P f=f_{1}(y)=(f(x), \Delta K(x, y)), \quad \text { for any } f \in \mathfrak{S} .
$$

The kernel $K$ corresponds to the identity $I$ and following (2) we have

$$
K(x, y)=\sum_{k} \Delta_{k} K(x, y)
$$

for any decomposition $\left\{\Delta_{k}\right\}$ of the real axis. The series in (8) converges absolutely. In fact, following (2), the series $K(y, z)=I K(y, z)=\sum \Delta_{k} P K(y, z)$ $=\sum\left(K(x, z), \Delta_{k} K(x, y)\right)_{x}=\sum \Delta_{k} K(y, z)$ as function of $y$ is strongly convergent. It converges then in the ordinary sense for every $y$, in particular for $y=z$. Thus, $K(z, z)=\sum \Delta_{k} K(z, z)<\infty, \Delta_{k} K(z, z) \geqq 0$. Consequently

$$
\begin{aligned}
\sum\left|\Delta_{k} K(x, y)\right| & \leqq \sum\left(\Delta_{k} K(x, x)\right)^{1 / 2}\left(\Delta_{k} K(y, y)\right)^{1 / 2} \\
& \leqq\left[\sum \Delta_{k} K(x, x) \cdot \sum \Delta_{k} K(y, y)\right]^{1 / 2} .
\end{aligned}
$$

If the resolution of identity $\left\{P_{\lambda}\right\}$ has a discrete spectrum $\left\{\theta_{k}\right\}$ and if the r.k. of $\delta_{\theta_{k}} \mathfrak{S}$ is denoted by $\delta_{\theta_{k}} K$, then we have again an absolutely convergent representation 


$$
K(x, y)=\sum_{k} \delta_{\theta_{k}} K(x, y) .
$$

An especially important case which is most often applied is one where the spectrum is simple. Then the subspaces $\delta_{\theta_{k}} \mathfrak{S}$ are one-dimensional and each is generated by a function $g_{k}(x)$ which we can suppose normalized, $\left\|g_{k}\right\|=1$. The functions $g_{k}(x)$ form a complete orthonormal system in $\mathfrak{S}$. The kernels $\delta_{\theta_{k}} K(x, y)$ are given by $g_{k}(x) \overline{g_{k}(y)}$, and (9) takes the form of the well known development of the kernel in an orthonormal complete system

$$
K(x, y)=\sum_{k} g_{k}(x) \overline{g_{k}(y)}
$$

which for a long time was taken as a basis of the definition of a r.k.

Suppose now that the resolution of identity $\left\{P_{\lambda}\right\}$ is given by a system of functions $f_{n}(\lambda) \equiv f_{n}(x, \lambda)$ satisfying (a), (b), and ( $\left.c^{\prime}\right)$. Following (4) we define for $u=K(x, y)$ (considered as function of $x$ ), the functions

$$
\Phi_{n}(y, \lambda)=\lim _{\Delta \rightarrow \lambda} \frac{\left(K(x, y), \Delta f_{n}\right)}{\Delta \mu_{n}}=\lim \frac{\overline{f_{n}\left(y, \lambda^{\prime \prime}\right)}-\overline{f_{n}\left(y, \lambda^{\prime}\right)}}{\mu_{n}\left(\lambda^{\prime \prime}\right)-\mu_{n}\left(\lambda^{\prime}\right)}=\frac{\overline{d f_{n}(y, \lambda)}}{d \mu_{n}(\lambda)} .
$$

From (6) and (5) we then obtain

$$
\begin{aligned}
K(y, z)=(K(x, z), K(x, y)) & =\sum_{n} \int_{-\infty}^{\infty} \Phi_{n}(y, \lambda) \overline{\Phi_{n}(z, \lambda)} d \mu_{n}, \\
K(y, y) & =\sum_{n} \int_{-\infty}^{\infty}\left|\Phi_{n}(y, \lambda)\right|^{2} d \mu_{n} .
\end{aligned}
$$

The series and the integrals in (12) are absolutely convergent because of (13).

The function $\Phi_{n}(y, \lambda)$ is in general defined for each $y$ only almost everywhere in $\lambda$ in the sense of the measure $\mu_{n}$. Nevertheless, in most applications it turns out to be a continuous function of $\lambda$. In spite of this, $\Phi_{n}\left(y, \lambda_{0}\right)$, as function of $y$ for a fixed $\lambda_{0}$, will not in general belong to $\$$.

11. Operators in spaces with reproducing kernels $\left({ }^{9}\right)$. In a class $F$ forming a Hilbert space with a r.k. $K$, the bounded operators admit of an interesting representation.

The notation $L_{x} K(x, y)$ indicates that the operator is applied to $K(x, y)$ as function of $x$ and that the resulting function is considered as function of $x$ (but it will depend also on $y$ which will act in the transformation as a parameter). It is then clear what is meant by $L_{z} \overline{K(x, z)}, L_{x} L_{z}^{\prime} \overline{K(x, z)}$, and so on. The notation $L f(x)$ is clear and we may also write $L f\left(x_{0}\right)$ if $x_{0}$ is a particular value of $x$. Consider the adjoint operator $L^{*}$ (that is, the operator for which $(L f, g)$ $\left.=\left(f, L^{*} g\right)\right)$. Take the transform

(9) The developments of this section are closely related with the work and ideas of E. H. Moore. 


$$
\Lambda(x, y)=L_{x}^{*} K(x, y) .
$$

$\Lambda$ is a function of the two points $x, y$. As function of $x$ it belongs to $F$. Take then for any $f \in F$ the scalar product $(f(x), \Lambda(x, y))_{x}=\left(f(x), L_{x}^{*} K(x, y)\right)_{x}$ $=(L f(x), K(x, y))_{x}=L f(y)$,

$$
L f(y)=(f(x), \Lambda(x, y)) .
$$

In this way, to each bounded operator there corresponds a kernel $\Lambda(x, y)$ which for every $y$, as function of $x$, belongs to $F$. The operator is represented in terms of the kernel by formula (2).

Let us now find the kernel $\Lambda^{*}(x, y)$ corresponding to the adjoint operator $L^{*}$. We have $\left(L^{*}\right)^{*}=L$ and thus

$$
\begin{aligned}
\left(L_{x} K(x, z), K(x, y)\right) & =\left(K(x, z), L_{x}^{*} K(x, y)\right), \\
\left(\Lambda^{*}(x, z), K(x, y)\right) & =\overline{(\Lambda(x, y), K(x, z)),} \\
\Lambda^{*}(y, z) & =\overline{\Lambda(z, y) .}
\end{aligned}
$$

It is clear that to $L_{1}+L_{2}$ or $\alpha L$ correspond $\Lambda_{1}+\Lambda_{2}$ and $\bar{\alpha} \Lambda$ respectively. We shall now find the kernel $\Lambda$ corresponding to the composition $L=L_{1} L_{2}$. Since $\left(L_{1} L_{2}\right)^{*}=L_{2}^{*} L_{1}^{*}$, we have

$$
\begin{array}{rlr}
\Lambda(y, z) & =\left(L_{1} L_{2}\right)_{y}^{*} K(y, z)=L_{2 y}^{*} L_{1 y}^{*} K(y, z) \\
& =L_{2 y}^{*} \Lambda_{1}(y, z)=\left(\Lambda_{1}(x, z), \Lambda_{2}^{*}(x, y)\right)=\left(\Lambda_{1}(x, z), \overline{\Lambda_{2}(y, x)}\right) \\
\Lambda(y, z) & =\left(\Lambda_{1}(x, z), \overline{\Lambda_{2}(y, x)}\right) \quad \text { for } L=L_{1} L_{2} .
\end{array}
$$

Let us note the following properties resulting immediately from (1)-(4):

$$
\begin{aligned}
\left((f(x), \Lambda(x, y))_{x}, g(y)\right)_{y} & =\left(f(x),(g(y), \overline{\Lambda(x, y)})_{y}\right)_{x} \\
& =\left(f(x), \overline{\left.(\overline{(x, y)}, g(y))_{y}\right)_{x}}\right.
\end{aligned}
$$

(6) The symmetry of $L$ is equivalent to the hermitian symmetry of $\Lambda$ :

$$
\Lambda(x, y)=\overline{\Lambda(y, x)} \text {. }
$$

We prove now the following property:

The operator $L$ is positive if and only if $\Lambda$ is a p. matrix.

In fact, $L$ positive means that for every $f \in F,(L f, f) \geqq 0$. For $f=\sum_{1}^{n} \zeta_{k} K\left(x, y_{k}\right)$ we then get

$$
\begin{aligned}
\sum_{i} \sum_{i} \zeta_{i} \bar{\zeta}_{j}\left(L_{x} K\left(x, y_{i}\right), K\left(x, y_{j}\right)\right) \\
=\sum \sum \zeta_{i} \bar{\zeta}_{j}\left(\Lambda^{*}\left(x, y_{i}\right), K\left(x, y_{j}\right)\right)=\sum \sum \Lambda^{*}\left(y_{j}, y_{i}\right) \zeta_{i} \zeta_{j} \\
=\sum \sum \overline{\Lambda\left(y_{i}, y_{j}\right)} \zeta_{i} \bar{\zeta}_{j}>0 .
\end{aligned}
$$


This proves that $\bar{\Lambda}$ and thus $\Lambda$ is also a p. matrix. It also proves the well known fact that a positive operator is always symmetric.

If now $\Lambda$ is a p. matrix, we see that $(L f, f) \geqq 0$ will be satisfied for all $f$ of the form $\sum_{1}^{n} \zeta_{k} K\left(x, y_{k}\right)$. As these functions form a dense set in $F$, every function $f \in F$ may be approximated by them and we get $(L f, f) \geqq 0$ by a passage to the limit.

In generalizing the notation of $\S 7$ we shall write $\Lambda_{1} \ll \Lambda_{2}$ or $\Lambda_{2} \ll \Lambda_{1}$ for any two kernels if $\Lambda_{2}-\Lambda_{1}$ is a p. matrix.

TheOREM I. For an arbitrary kernel $\Lambda(x, y)$, hermitian symmetric (that is, $\Lambda(x, y)=\overline{\Lambda(y, x))}$, the necessary and sufficient condition that it correspond to $a$ bounded symmetric operator with lower bound $\geqq m>-\infty$ and upper bound $\leqq M<+\infty$ is that $m K \ll \Lambda \ll M K$.

Proof. Necessity. If $L$ is the corresponding symmetric operator with bounds not less than $m$ and not greater than $M$, we have

$$
m(f, f) \leqq(L f, f) \leqq M(f, f) \quad \text { for every } f \in F .
$$

It follows that $((L-m I) f, f) \geqq 0$ and $((M I-L) f, f) \geqq 0$, that is, the operators $L-m I$ and $M I-L$ are positive. Therefore, from (7) we obtain that $\Lambda-m K$ and $M K-\Lambda$ are p. matrices.

Suffciency. The condition of the theorem is clearly equivalent to

$$
0 \ll \frac{1}{M-m}(\Lambda-m K) \ll K \text {. }
$$

This means that the kernel $K_{1}=(1 /(M-m)(\Lambda-m K)$ is a p. matrix and is $\ll K$. Therefore it is a reproducing kernel of a class $F_{1}$ with the norm \|\|$_{1}$ and following Theorem I, $\$ 7, F_{1} \subset F$ and $\left\|f_{1}\right\|_{1} \geqq\left\|f_{1}\right\|$ for $f_{1} \in F_{1}$. Then, as in Theorem III, §7, the operator

$$
L_{1} f(y)=\left(f(x), K_{1}(x, y)\right)
$$

is a positive operator in $F$ with a bound not greater than 1 , that is,

$$
0 \leqq\left(L_{1} f, f\right) \leqq(f, f) \text {. }
$$

This operator corresponds to $K_{1}(x, y)$ by its definition. Consequently, to $\Lambda=(M-m) K_{1}+m K$ there corresponds the operator $L=(M-m) L_{1}+m I$ and the last inequalities give

$$
\begin{aligned}
& m(f, f) \leqq(m I f, f)+\left((M-m) L_{1} f, f\right) \leqq M(f, f), \\
& m(f, f) \leqq(L f, f) \leqq M(f, f) .
\end{aligned}
$$

An arbitrary kernel $\Lambda$ is representable in a unique way in the form

$$
\Lambda=\Lambda_{1}+i \Lambda_{2}, \Lambda_{1} \text { and } \Lambda_{2} \text { hermitian symmetric. }
$$

Namely, we have 


$$
\begin{aligned}
& \Lambda_{1}(x, y)=\frac{1}{2}(\Lambda(x, y)+\overline{\Lambda(y, x)}), \\
& \Lambda_{2}(x, y)=\frac{1}{2 i}(\Lambda(x, y)-\overline{\Lambda(y, x)} .
\end{aligned}
$$

The necessary and sufficient condition in order that $\Lambda$ correspond to a bounded operator is, clearly, that $\Lambda_{1}$ and $\Lambda_{2}$ correspond to such operators. To the last kernels we can apply Theorem $I$.

We now consider convergence of operators. The three simplest notions of limit for bounded operators are the following: the weak limit, w. $\lim _{n=\infty} L_{n}=L$, if $L_{n} u$ converges weakly to $L u$ for every $u \in F$; the strong limit, str. $\lim L_{n}=L$, if $L_{n} u$ converges strongly to $L u$; the uniform limit, un. $\lim L_{n}=L$, if $\left\|L_{n}-L\right\|$ $\rightarrow 0$, where \|\| for operators denotes their bound.

It is clear that weak convergence follows from strong convergence and that strong convergence follows from the uniform one.

It is known that w. $\lim L_{n}=L$ involves the boundedness of all $\left\|L_{n}\right\|$ and the inequality $\|L\| \leqq \lim$ inf. $\left\|L_{n}\right\|$.

THEOREM II. If $L=\mathrm{w} . \lim L_{n}$, then for the corresponding kernels we have $\Lambda(x, y)=\lim \Lambda_{n}(x, y)$ for every $x, y$ in $E$. If $L=$ un. $\lim L_{n}$, then $\Lambda_{n}$ converges uniformly to $\Lambda$ in every set of couples $(x, y)$ for which $K(x, x)$ and $K(y, y)$ are uniformly bounded. from

The first part follows immediately, by the definition of weak convergence

$$
\begin{aligned}
\Lambda(x, y) & =(\Lambda(z, y), K(z, x))_{z}=\left(L_{z}^{*} K(z, y), K(z, x)\right)_{z} \\
& =\left(K(z, y), L_{z} K(z, y)\right)_{z}=\lim \left(K(z, y), L_{n z} K(z, x)\right)_{z}=\lim \Lambda_{n}(x, y) .
\end{aligned}
$$

The second part follows easily from

$$
\begin{aligned}
\left|\Lambda(x, y)-\Lambda_{n}(x, y)\right| & =\left|\left(K(z, y),\left(L-L_{n}\right)_{z} K(z, x)\right)\right| \\
& \leqq\|K(z, y)\|_{z}\left\|\left(L-L_{n}\right)_{z} K(z, x)\right\|_{z} \\
& \leqq\|K(z, y)\| z\left\|L-L_{n}\right\|\|K(z, x)\|_{z} \\
& =\left\|L-L_{n}\right\|(K(x, x) K(y, y))^{1 / 2}
\end{aligned}
$$

Consider now two orthonormal complete systems in $F,\left\{g_{m}^{\prime}\right\}$ and $\left\{g_{n}^{\prime \prime}\right\}$ (in particular we may have $\left.g_{m}^{\prime}=g_{m}^{\prime \prime}\right)$. The double system $\left\{g_{m}^{\prime}(x) \overline{g_{n}^{\prime \prime}(y)}\right\}$ is a complete orthonormal system in the direct product $F \otimes \bar{F}$.

If $\Lambda(x, y)$ belongs to the direct product we know that it is representable by an absolutely convergent double series

$$
\Lambda(x, y)=\sum_{m, n} \alpha_{m n} g_{m}^{\prime}(x) \overline{g_{n}^{\prime \prime}(y)}
$$


where the coefficients $\alpha_{m n}$ satisfy $\sum_{m, n}\left|\alpha_{m n}\right|^{2}<\infty$ and are given by

$$
\alpha_{m n}=\left(g_{n}^{\prime \prime}(y),\left(g_{m}^{\prime}(x), \Lambda(x, y)\right)_{x}\right)_{y}=\left(g_{n}^{\prime \prime}(y), L g_{m}^{\prime}(y)\right) .
$$

THEOREM III. For every bounded operator $L$, the series in (10) with coefficients given by (11) is convergent for every $x$ and $y$ in the sense

$$
\Lambda(x, y)=\lim _{p, q=\infty} \sum_{m=1}^{p} \sum_{n=1}^{q} \alpha_{m n} g_{m}^{\prime}(x) \overline{g_{n}^{\prime \prime}(y)} .
$$

The $\Lambda(x, y)$ belonging to the direct product $F \otimes \bar{F}$ correspond to operators with finite norm.

Let $P_{p}^{\prime}$, and $P_{q}^{\prime \prime}$. be the projections on the subspaces generated by $g_{1}^{\prime}, g_{2}^{\prime}, \cdots, g_{p}^{\prime}$ and $g_{1}^{\prime \prime}, g_{2}^{\prime \prime}, \cdots, g_{q}^{\prime \prime}$. It is clear that str. $\lim _{p=\infty} P_{p}^{\prime}=I$, str. $\lim _{q=\infty} P_{g}^{\prime \prime}=I$. Consequently, for any $u, v$ in $F$

$$
\lim _{p, q=\infty}\left(P_{q}^{\prime \prime} v, L P_{p}^{\prime} u\right)=(v, L u) .
$$

If we take now $v=K(z, y)$ and $u=K(z, x)$ as functions of $z$, we get $P_{q}^{\prime \prime} v$ $=P_{q z}^{\prime \prime} K(z, y)=\sum_{1}^{q} g_{n}^{\prime \prime}(z) \overline{g_{n}^{\prime \prime}(y)}, P_{p}^{\prime} u=\sum_{1}^{p} g_{m}^{\prime}(z) g_{m}^{\prime}(x)$ and (11) and (13) then lead directly to (12).

The norm of an operator $L$ is given by $\mathfrak{N}(L)=\cdot \sum_{m-1}^{\infty}\left\|L g_{m}\right\|^{2}$ for any orthonormal complete system $\left\{g_{n}\right\}$. It is independent of the choice of this system and may be finite or infinite. From (11) it is clear that

$$
L g_{m}^{\prime}(y)=\sum_{n=1}^{\infty} \overline{\alpha_{m n}} g_{n}^{\prime \prime}(y)
$$

by development in the system $\left\{g_{n}^{\prime \prime}\right\}$. Consequently, $\left\|L g_{m}^{\prime}(y)\right\|^{2}=\sum_{n=1}^{\infty}\left|\alpha_{m n}\right|^{2}$ and $\mathfrak{R}(L)=\sum_{m=1}^{\infty} \sum_{n=1}^{\infty}\left|\alpha_{m n}\right|^{2}$ which proves the second part of our theorem.

12. The reproducing kernel of a sum of two closed subspaces. Let $F$ be a class with a r.k. $K$. We know that the r.k.'s of closed subspaces of $F$ correspond to the projections on these subspaces.

The problem of expressing the r.k. of the sum $F_{1} \oplus F_{2}$ of two closed subspaces in terms of the r.k.'s $K_{1}$ and $K_{2}$ of these subspaces is therefore reduced to the problem of expressing the projection $P$ on $F_{1} \oplus F_{2}$ in terms of the projections $P_{1}$ and $P_{2}$ on $F_{1}$ and $F_{2}$.

In order to obtain this we shall at first prove the identity

$$
\left[\left(P-P_{1}\right)\left(P-P_{2}\right)\right]^{m}
$$

$$
\left.=P-\sum_{k=1}^{m} P_{1}\left(P_{2} P_{1}\right)^{k-1}+P_{2}\left(P_{1} P_{2}\right)^{k-1}-\left(P_{2} P_{1}\right)^{k}-\left(P_{1} P_{2}\right)^{k}\right]-\left(P_{2} P_{1}\right)^{m} \text {. }
$$

We shall use the known properties of projections, namely: $P_{1}=P_{1} P=P P_{1}$ $=P_{1}^{2}, P_{2}=P_{2} P=P P_{2}=P_{2}^{2}$, 


$$
P_{1}\left(P-P_{1}\right)=\left(P-P_{1}\right) P_{1}=0, \quad P_{2}\left(P-P_{2}\right)=\left(P-P_{2}\right) P_{2}=0 .
$$

Then, denoting the expression $\left(P-P_{1}\right)\left(P-P_{2}\right)$ by $Q$ we have

$$
\begin{aligned}
Q^{k} P_{1} & =Q^{k-1}\left(P-P_{1}\right)\left(P-P_{2}\right) P_{1}=Q^{k-1}\left(P-P_{1}\right)\left(P_{1}-P_{2} P_{1}\right) \\
& =-Q^{k-1} P_{2} P_{1}+Q^{k-1} P_{1} P_{2} P_{1} .
\end{aligned}
$$

If $k>1$, the first term is $-Q^{k-2}\left(P-P_{1}\right)\left(P-P_{2}\right) P_{2} P_{1}=0$, and we have

$$
Q^{k} P_{1}=Q^{k-1} P_{1} P_{2} P_{1}, \quad k>1 \text {. }
$$

For $k=1$ we obtain

$$
Q P_{1}=-P_{2} P_{1}+P_{1} P_{2} P_{1}
$$

Finally, we obtain by induction

$$
Q^{k} P_{1}=-\left(P_{2} P_{1}\right)^{k}+P_{1}\left(P_{2} P_{1}\right)^{k}
$$

Further, we have

$$
Q=\left(P-P_{1}\right)\left(P-P_{2}\right)=P-P_{1}-P_{2}+P_{1} P_{2} .
$$

This gives

$$
Q^{n}=Q^{n-1}\left(P-P_{1}-P_{2}+P_{1} P_{2}\right)=Q^{n-1} P-Q^{n-1} P_{1}-Q^{n-1} P_{2}+Q^{n-1} P_{1} P_{2}
$$

Since $Q^{n-1} P=Q^{n-1}, Q^{n-1} P_{2}=0$, we get from (2)

$$
\begin{aligned}
Q^{n}= & Q^{n-1}-Q^{n-1} P_{1}+Q^{n-1} P_{1} P_{2} \\
= & Q^{n-1}-\left[-\left(P_{2} P_{1}\right)^{n-1}+P_{1}\left(P_{2} P_{1}\right)^{n-1}\right] \\
& +\left[-\left(P_{2} P_{1}\right)^{n-1} P_{2}+P_{1}\left(P_{2} P_{1}\right)^{n-1} P_{2}\right] \\
= & Q^{n-1}+\left(P_{2} P_{1}\right)^{n-1}-P_{1}\left(P_{2} P_{1}\right)^{n-1}-P_{2}\left(P_{1} P_{2}\right)^{n-1}+\left(P_{1} P_{2}\right)^{n} .
\end{aligned}
$$

This expression is valid for $n \geqq 2$. Adding these equations side by side for $n=m, m-1, \cdots, 2$, and using the formula for $Q$ given in (3), we obtain the required formula for $Q^{m}$. This formula may be written in the form

$$
\begin{aligned}
P= & {\left[\left(P-P_{1}\right)\left(P-P_{2}\right)\right]^{m}+\left(P_{2} P_{1}\right)^{m} } \\
& +\sum_{k=1}^{m}\left[P_{1}\left(P_{2} P_{1}\right)^{k-1}+P_{2}\left(P_{1} P_{2}\right)^{k-1}-\left(P_{2} P_{1}\right)^{k}-\left(P_{1} P_{2}\right)^{k}\right] .
\end{aligned}
$$

We shall prove now that for $m \rightarrow \infty,\left(P_{2} P_{1}\right)^{m}$ converges strongly to the projection $P_{0}$ on the intersection $F_{0}$ of $F_{1}$ and $F_{2}$. In order to do so we shall consider the operator $L=P_{2} P_{1}$ in the space $F_{2}$.

In this space $L$ is a positive operator (and therefore symmetric) with bound not greater than 1 . In fact, for $u \in F_{2}$

$$
\begin{aligned}
\left(P_{2} P_{1} u, P_{2} P_{1} u\right) & =\left\|P_{2} P_{1} u\right\|^{2} \leqq\left\|P_{1} u\right\|^{2}=\left(P_{1} u, P_{1} u\right)=\left(P_{1} u, u\right)=\left(P_{1} u, P_{2} u\right) \\
& =\left(P_{2} P_{1} u, u\right)=\left\|P_{1} u\right\|^{2} \leqq\|u\|^{2},
\end{aligned}
$$




$$
0 \leqq(L u, L u) \leqq(L u, u) \leqq(u, u)
$$

Consider now for any $f \in F$ the sequence $\left\{L^{k} f\right\}$. For $k \geqq 1, L^{k} f=P_{2} P_{1} L^{k-1} f$ $\in F_{2}$. Putting $u=L^{k} f$ in (5), we get

$$
\begin{aligned}
& 0 \leqq\left(L^{k+1} f, L^{k+1} f\right) \leqq\left(L^{k+1} f, L^{k} f\right) \leqq\left(L^{k} f, L^{k} f\right), \\
& 0 \leqq\left(L^{2 k+1} f, L f\right) \leqq\left(L^{2 k} f, L f\right) \leqq\left(L^{2 k-1} f, L f\right) .
\end{aligned}
$$

Consequently the sequence $\left.\left\{L^{n} f, L f\right)\right\}$ is a decreasing sequence of positive numbers and therefore it is convergent. This gives

$$
\begin{aligned}
\lim _{m, n=\infty}\left\|L^{m} f-L^{n} f\right\|^{2}= & \lim \left[\left(L^{m} f, L^{m} f\right)-\left(L^{m} f, L^{n} f\right)\right. \\
& \left.-\left(L^{n} f, L^{m} f\right)+\left(L^{n} f, L^{n} f\right)\right] \\
= & \lim \left[\left(L^{2 m-1} f, L f\right)-2\left(L^{m+n-1} f, L f\right)+\left(L^{2 n-1} f, L f\right)\right]=0
\end{aligned}
$$

and thus $L^{m} f$ converges strongly. This means that $L^{m}$ converges strongly to some bounded operator $P_{0}$. We have further $L^{m+1} f=L L^{m} f=L^{m} L f$, which, for $m \rightarrow \infty$, gives

$$
L P_{0} f=P_{0} f=P_{0} L f .
$$

Therefore $L^{m} P_{0} f=P_{0} f$ and $P_{0}^{2} f=\lim L^{m} P_{0} f=P_{0} f$. In the subspace $F_{2}, P_{0}$ as a limit of symmetric operators is symmetric. Together with $P_{0}^{2} f=P_{0} f$ it shows that in $F_{2}$ the operator $P_{0}$ is a projection. It is the projection on the subspace of all $P_{0} f$. From (6) we get $\left\|P_{2} P_{1} P_{0} f\right\| \leqq\left\|P_{1} P_{0} f\right\| \leqq\left\|P_{0} f\right\|=\left\|P_{2} P_{1} P_{0} f\right\|$. Consequently $\left\|P_{2} P_{1} P_{0} f\right\|=\left\|P_{1} P_{0} f\right\|=\left\|P_{0} f\right\|, \quad P_{2} P_{1} P_{0} f=P_{1} P_{0} f=P_{0} f \quad$ and $P_{0} f \in F_{0}=F_{1} \cdot F_{2}$. Inversely if $u \in F_{1} \cdot F_{2}$, then $L u=P_{2} P_{1} u=u$ and $P_{0} u$ $=\lim L^{n} u=u$.

Thus, in $F_{2}, P_{0}$ is the projection on $F_{0}$. Then for any $f \in F$, we have by (6) $P_{0} f=P_{0} L f=P_{0} P_{2} P_{1} f=$ projection of $f$ on $F_{0}$.

In our formula (4), besides the series $\sum$ and the term $\left(P_{2} P_{1}\right)^{m}$ we have still the expression $\left(P-P_{1}\right)\left(P-P_{2}\right)^{m} . P-P_{1}$ is the projection on $F^{\prime} \ominus F_{1}$, and $P \ominus P_{2}$ is the projection on $F^{\prime} \ominus F_{2}$, if we denote $F_{1} \oplus F_{2}$ by $F^{\prime}$. Consequently for $m \rightarrow \infty$, the last expression converges strongly to the projection on the intersection of $\left(F^{\prime} \ominus F_{1}\right)$ and $\left(F^{\prime} \ominus F_{2}\right)$. But this intersection is reduced to the element zero because were there in it any element $u \neq 0$, it would belong to $F^{\prime}$ and would be orthogonal to $F_{1}$ as well as to $F_{2}$. Thus, $u$ would be orthogonal to $F_{1} \oplus F_{2}=F^{\prime}$ which is impossible.

In this way we finally obtain the desired formula for the projection $P$ :

$$
P=P_{0}+\sum_{k=1}^{\infty}\left[P_{1}\left(P_{2} P_{1}\right)^{k-1}+P_{2}\left(P_{1} P_{2}\right)^{k-1}-\left(P_{2} P_{1}\right)^{k}-\left(P_{1} P_{2}\right)^{k}\right] \text {. }
$$

The subspace $F_{1} \oplus F_{2}$ is defined as the closure of the subspace $F_{1} \dot{+} F_{2}$ composed of all sums $f_{1}+f_{2}, f_{1} \in F_{1}, f_{2} \in F_{2}$. In general $F_{1}+F_{2}$ is not a closed subspace. 
Formula (7) is especially advantageous when $F_{1} \dot{+} F_{2}$ is closed and thus equal to $F^{\prime}=F_{1} \oplus F_{2}$.

Let us analyze this case more in detail. It will be convenient to make the non-essential assumption that

$$
F_{0}=F_{1} \cdot F_{2}=(0), \text { that is, } P_{0}=0 .
$$

The angle between two elements (vectors) $f_{1} \neq 0, f_{2} \neq 0$ is given by $\cos \alpha=\operatorname{Re}\left(f_{1}, f_{2}\right) /\left\|f_{1}\right\|\left\|f_{2}\right\|$. The minimal angle $\phi, 0 \leqq \phi \leqq \pi / 2$, between $F_{1}$ and $F_{2}$ is given by $\left({ }^{10}\right)$

$$
\cos \phi=\text { l.u.b. } \operatorname{Re} \frac{\left(f_{1}, f_{2}\right)}{\left\|f_{1}\right\|\left\|f_{2}\right\|} \quad \text { for } 0 \neq f_{1} \in F_{1}, 0 \neq f_{2} \in F_{2} .
$$

It is easily seen that, for $f_{1} \in F_{1}, f_{2} \in F_{2}$,

$$
\begin{array}{r}
\left|\left(f_{1}, f_{2}\right)\right| \leqq\left\|f_{1}\right\|\left\|f_{2}\right\| \cos \phi, \\
\left\|P_{1} f_{2}\right\| \leqq\left\|f_{2}\right\| \cos \phi, \quad \quad\left\|P_{2} f_{1}\right\| \leqq\left\|f_{1}\right\| \cos \phi, \\
\left\|f_{1}+f_{2}\right\| \geqq\left\|f_{1}\right\| \sin \phi, \quad\left\|f_{1}+f_{2}\right\| \geqq\left\|f_{2}\right\| \sin \phi .
\end{array}
$$

In (12), $\sin \phi$ is the greatest constant $c \geqq 0$ for which an inequality of type $\left\|f_{1}+f_{2}\right\| \geqq c\left\|f_{1}\right\|$ is true. By a theorem of $\mathrm{H}$. Kober [1] such inequality with $c>0$ is necessary and sufficient in order that $F_{1} \dot{+} F_{2}$ be closed.

Consequently, we shall know that $F^{\prime}=F_{1} \dot{+} F_{2}$ if we prove an inequality

$$
\left\|f_{1}+f_{2}\right\| \geqq c\left\|f_{1}\right\| \text {, }
$$

with any $c>0$. Such a constant is necessarily less than or equal to 1 and it gives always an evaluation of the minimal angle $\phi$ :

$$
\sin \phi \geqq c>0 \text {. }
$$

The angle $\phi$ being positive, the inequalities (11) show that the bounds of the operators $\left(P_{2} P_{1}\right)^{n}$ in $F_{2}$ or $\left(P_{1} P_{2}\right)^{n}$ in $F_{1}$ are not greater than $\cos ^{2 n} \phi$. Formula (7) may now be written in the form

$$
\begin{aligned}
P= & \left(P_{1}-P_{1} P_{2}+P_{1} P_{2} P_{1}-P_{1} P_{2} P_{1} P_{2}+\cdots\right) \\
& +\left(P_{2}-P_{2} P_{1}+P_{2} P_{1} P_{2}-P_{2} P_{1} P_{2} P_{1}+\cdots\right)
\end{aligned}
$$

and the two series are uniformly convergent to the operators $Q_{1}$ and $Q_{2}$ which give the decomposition of $f \in F_{1}+F_{2}$ in $f=Q_{1} f+Q_{2} f, Q_{1} f \in F_{1}, Q_{2} f \in F_{2}$.

It should be remarked that the decomposition of the series in (7) into the two series (15) is not possible when $\phi=0$, as the operators $Q_{1}$ and $Q_{2}$ are then unbounded.

When the series in (15) are used for computation it is very easy to get

$\left({ }^{10}\right)$ The notion of a minimal angle between two subspaces seems to have been first introduced by K. Friedrichs [1]. 
estimates for the remainder. Usually we shall want to compute, for $f$ and $g$ in $F$, the value of $(f, P g)=\left(f, Q_{1} g\right)+\left(f, Q_{2} g\right)$. It is clear that when we stop in the series of $Q_{1}$ at the $n$th term $P_{1}^{(n)}=(-1)^{n-1} P_{1} P_{2} P_{1} \cdots$, then the remain$\operatorname{der} R_{1}^{(n)}$ of this series will be given by

$$
R_{1}^{(n)}=-P_{1}^{(n)} Q_{2} \text { for odd } n, \quad R_{1}^{(n)}=-P_{1}^{(n)} Q_{1} \text { for even } n .
$$

We have similar developments for the second series defining $Q_{2}$. Consequently, the error in $\left(f, Q_{1} g\right)$ (for example when $n$ is odd) is given by $\left(f, R_{1}^{(n)} g\right.$ ) $=-\left(P_{1}^{(n) *} f, Q_{2} g\right)$

$$
\left|\left(f, R_{1}^{(n)} g\right)\right| \leqq\left\|P_{1}^{(n) *} f\right\|\left\|Q_{2} g\right\| .
$$

By (12) we have $\left\|Q_{2} g\right\| \leqq(1 / \sin \phi)\|g\|$. As $P_{1}^{(n)}$ is already computed, $P_{1}^{(n) *}$ is known also and we can compute $\left\|P_{1}^{(n) * f}\right\|$. This will give quite a precise evaluation of the error. Without knowing $P_{1}^{(n)}$ we can evaluate $\left\|P_{1}^{(n)} *_{f}\right\|$ $\leqq\|f\| \cos ^{n-1} \phi$.

Even in case $\phi=0$ we could still evaluate the error in $(f, P g)$ if $Q_{1} g$ and $Q_{2} g$ exist and if we can evaluate their norms.

Still another evaluation of error (preferable as an a priori evaluation), in the case $\phi>0$, is obtained directly from (4):

$$
P-\sum_{k=1}^{m}[]=\left[\left(P-P_{1}\right)\left(P-P_{2}\right)\right]^{m}+\left(P_{2} P_{1}\right)^{m} .
$$

It can be proved that the minimal angle of $F^{\prime} \ominus F_{1}$ and $F^{\prime} \ominus F_{2}$ is the same as between $F_{1}$ and $F_{2}$. Consequently

$$
\begin{gathered}
\left\|\left[\left(P-P_{1}\right)\left(P-P_{2}\right)\right]\right\| m \leqq \cos ^{2 m-1} \phi, \quad\left\|\left(P_{2} P_{1}\right)^{m}\right\| \leqq \cos ^{2 m-1} \phi, \\
\left\|P-\sum_{k=1}^{m}[]\right\|<2 \cos ^{2 m-1} \phi,
\end{gathered}
$$

where \| $\mid$ signifies bounds of operators.

In case of a sum of more than two subspaces $F^{\prime}=F_{1} \oplus F_{2} \oplus F_{3} \oplus \cdots$ we can still express the projection on $F^{\prime}$ in terms of projections on $F_{1}, F_{2}, \cdots$, but the formula will be much more complicated than in the case of two subspaces and for this reason may not be as valuable.

Let us consider now the translation of our formulas in terms of the reproducing kernels $K_{1}, K_{2}$, and $K^{\prime}$ of the classes $F_{1}, F_{2}$, and $F^{\prime}=F_{1} \oplus F_{2}$. We shall suppose that the classes $F_{1}$ and $F_{2}$ have no function $\neq 0$ in common, that is, $F_{1} \cdot F_{2}=(0)$.

To the projections $P_{1}, P_{2}, P$ there correspond (in the sense of $\$ 11$ ) the kernels $K_{1}, K_{2}$ and $K^{\prime}$. To each term in the series (7) or (15) there corresponds a kernel given by the following table of correspondence

$$
P \leftrightarrow K^{\prime}(x, y), \quad P_{1} \leftrightarrow K_{1}(x, y), \quad P_{2} \leftrightarrow K_{2}(x, y),
$$




$$
\begin{aligned}
& P_{1} P_{2} \leftrightarrow \Lambda_{1}(x, y)=\left(K_{1}(z, y), K_{2}(z, x)\right)_{z}, \\
& P_{2} P_{1} \leftrightarrow \overline{\Lambda_{1}(y, x)}=\left(K_{2}(z, y), K_{1}(z, x)\right)_{z}, \\
&\left(P_{1} P_{2}\right)^{n} \leftrightarrow \Lambda_{n}(x, y), \quad\left(P_{2} P_{1}\right)^{n} \leftrightarrow \overline{\Lambda_{n}(y, x)},
\end{aligned}
$$

where

$$
\begin{gathered}
\Lambda_{n}(x, y)=\left(\Lambda_{1}(z, y), \overline{\Lambda_{n-1}(x, z)}\right)_{z}=\left(\Lambda_{n_{1}}(z, y), \overline{\Lambda_{n_{2}}(x, z)}\right)_{z}, \quad n_{1}+n_{2}=n, \\
P_{1}\left(P_{2} P_{1}\right)^{n} \leftrightarrow \Lambda_{n}^{\prime}(x, y)=\overline{\Lambda_{n}^{\prime}(y, x)}=\left(K_{1}(z, y), \overline{\left.\Lambda_{n}(z, x)\right)_{z},}\right. \\
P_{2}\left(P_{1} P_{2}\right)^{n} \leftrightarrow \Lambda_{n}^{\prime \prime}(x, y)=\overline{\Lambda_{n}^{\prime \prime}(y, x)}=\left(K_{2}(z, y), \overline{\Lambda_{n}(x, z)}\right)_{z} .
\end{gathered}
$$

Formula (15) can now be written in the form

$$
\begin{aligned}
K^{\prime}(x, y) & =\sum_{n=1}^{\infty}\left(\Lambda_{n-1}^{\prime}(x, y)+\Lambda_{n-1}^{\prime \prime}(x, y)-\Lambda_{n}(x, y)-\overline{\Lambda_{n}(y, x)}\right) \\
& =\sum_{n=1}^{\infty}\left(\Lambda_{n-1}^{\prime}(x, y)-\Lambda_{n}(x, y)\right)+\sum_{n=1}^{\infty}\left(\Lambda_{n-1}^{\prime \prime}(x, y)-\overline{\Lambda_{n}(y, x)}\right)
\end{aligned}
$$

If we use these series to compute $K^{\prime}(x, y)$ for given points $x$ and $y$, we shall represent it in the form

$$
K^{\prime}(x, y)=\left(K^{\prime}(z, y), P_{z} K^{\prime}(z, x)\right)=\left(K(z, y), P_{z} K(z, x)\right)
$$

and apply our evaluation of error to this form.

13. Final remarks in the general theory. In the present section we shall collect a number of shorter remarks about the nature of classes of functions with reproducing kernels and of their norms, and concerning some relations between the classes and their r.k.'s.

(A) Classes of functions for which a r.k. exists. (R.K.)-classes. Consider a set $E$ and a linear class $F$ of functions defined (and finite) everywhere in $E$. The problem which arises is to find under what circumstances we can define a norm in $F$ giving to $F$ the structure of a Hilbert space with a r.k. For abbreviation we shall call such classes of functions (R.K.)-classes.

THEOREM I. In order that the class $F$ (not necessarily linear) be contained in a (R.K.)-class it is necessary that there exist an increasing sequence of sets $E_{1} \subset E_{2} \subset \cdots, E=E_{1}+E_{2}+\cdots$, and for each $f \neq 0$ of $F$ a positive number $N(f)$, so that the functions $f(x) / N(f)$ be uniformly bounded in each $E_{n}$.

In fact if $F_{1}$ is the (R.K.)-class containing $F,\|\|_{1}$ and $K_{1}$ its norm and kernel, we define as $E_{n}$ the set of all $y \in E$ with $K_{1}(y, y) \leqq n$ and as $N(f)$ the norm $\|f\|_{1}$. Then, for each $y \in E_{n}$ and $f \in F \subset F_{1}$ we have $|f(y)|=\mid(f(x)$, $\left.K_{1}(x, y)\right)_{1} \mid \leqq\|f\|\left\|_{1} K_{1}(x, y)\right\|_{1}=N(f)\left(\left(K_{1}(y, y)\right)^{1 / 2}\right.$ and $|f(y)| / N(f) \leqq n^{1 / 2}$.

The necessary condition of Theorem $I$ is not always satisfied even for an enumerable sequence of functions. As an example, consider in the interval $E=(0,1)$ the sequence of functions $f_{n}(x)=1 /\left|x-r_{n}\right|$ for $x \neq r_{n}$ and $f_{n}\left(r_{n}\right)=0$. 
Here $\left\{r_{n}\right\}$ is a sequence of numbers everywhere dense in $E$. By an easy topological argument we prove that the sequence of functions $f_{n}$ does not satisfy the condition of Theorem I.

THEOREM II. For an enumerable sequence $\left\{f_{n}(x)\right\}$ the condition of Theorem $I$ is also sufficient in order that this sequence be contained in a (R.K.)-class.

In fact, consider an upper bound $M_{m}<\infty$ for $\left|f_{n}(x)\right| / N(f), n=1,2, \cdots$, $x \in E_{m}$. We write

$$
K(x, y)=\sum_{n=1}^{\infty} \frac{1}{2^{n} M_{n}^{2} N^{2}\left(f_{n}\right)} f_{n}(x) \overline{f_{n}(y)} .
$$

Clearly, the series is absolutely convergent and represents a p. matrix. Each term of it

$$
K_{n}(x, y)=\frac{1}{2^{n} M_{n}^{2} N^{2}\left(f_{n}\right)} f_{n}(x) \overline{f_{n}(y)}
$$

is also a p. matrix and $K_{n} \ll K$. Theorem I of $\$ 7$ gives then for the corresponding classes $F_{n} \subset F$. Obviously $F_{n}$ is the one-dimensional class generated by $f_{n}$. Therefore $f_{n} \in F$ and $\left\{f_{n}\right\} \subset F$.

The condition of Theorem I is certainly not sufficient in general. This may be shown by a simple set-theoretical argument. Let us consider namely the class $F_{b}$ of all bounded functions on $E$. We can then take $E_{n}=E, N(f)$ $=$ l.u.b. $|f(x)|$. If $\boldsymbol{N}$ is the power of $E$ then the power of a (R.K.)-class $F$ is $\mathrm{NO}_{0}$ (as the functions $K(x, y)=h_{y}(x)$ form a complete system in $F$ ). On the other hand, the power of the class $F_{b}$ is $=c^{\aleph}$ ( $c$ is the power of continuum) and for $\boldsymbol{N}>\boldsymbol{N}_{0}, \mathbf{c}^{\boldsymbol{N}}>\boldsymbol{N}^{\boldsymbol{N}_{0}}$.

(B) Convergence in classes with reproducing kernels. Consider a class $F$ with a r.k. $K$. We know that if $f_{n}$ converges strongly to $f$ in $F$, then it converges uniformly in every subset of $E$ where $K(x, x)$ is uniformly bounded. Therefore the sequence $\left\{f_{n}\right\}$ satisfies the condition

(1) $f_{n}(x) \rightarrow f(x)$ for every $x \in E$, the convergence being uniform in every set of an increasing sequence of sets $E_{1} \subset E_{2} \subset \cdots$ with $E=E_{1}+E_{2}+\cdots$.

Consider now the class $\Phi$ of all functions defined in $E$. In $\Phi$ we can introduce a notion of limit as follows:

$$
f(x)=\Phi-\lim f_{n}(x), \quad \text { if condition (1) is satisfied. }
$$

It is clear that in general the sequence of sets $E_{n}$ will depend on the sequence $\left\{f_{n}\right\}$. We can now formulate the following theorem.

THEOREM III. In every class $F$ with a $r . k$., the strong convergence of $f_{n}(x)$ to $f(x)$ involves $\Phi-\lim f_{n}(x)=f(x)$.

It should be noted that the weak convergence in $F$ does not involve in 
general the $\Phi$-convergence. But there are important cases where even weak convergence involves $\Phi$-convergence. Such cases were considered in $\$ 2,(5)$.

(C) Relations between (R.K.)-classes and corresponding norms and reproducing kernels. To a p. matrix there corresponds a uniquely determined class and norm, but to a (R.K)-class there correspond infinitely many norms giving to it the structure of a Hilbert space with a r.k. Consequently to a (R.K.)-class there correspond also infinitely many p. matrices which are r.k.'s of the class for convenient norms.

If the norm \|\| corresponds to a (R.K.)-class $F$, the norm \|\|$_{1}=c\|\cdot\|$, $c>0$, obviously also corresponds to $F$ and the corresponding r.k.'s $K$ and $K_{1}$ satisfy

$$
K_{1}(x, y)=\frac{1}{c^{2}} K(x, y) .
$$

In fact, the scalar product $(,)_{1}$ is clearly $=c^{2}($,$) and thus f(y)$ $=(f(x), K(x, y))=c^{2}\left(f(x),\left(1 / c^{2}\right) K(x, y)\right)=\left(f(x),\left(1 / c^{2}\right) K(x, y)\right)_{1}$.

We shall now have to apply an important theorem of S. Banach [1] in the theory of linear transformations.

Let $T$ be a linear transformation of a linear subspace $F^{\prime}$ of a complete space $F$ on a linear subspace $F_{1}^{\prime}$ of a complete space $F_{1}$. The subspaces $F^{\prime}$ and $F_{1}^{\prime}$, are not necessarily closed. The transformation $T$ is called closed if from $\left\{f_{n}\right\} \subset F^{\prime}, f_{n} \rightarrow f \in F$, and $T f_{n} \rightarrow f_{1} \in F_{1}$ follows $f \in F^{\prime}, f_{1} \in F_{1}^{\prime}$ and $T f=f_{1}$.

BANACH's THEOREM. If $T$ is a closed linear transformation of $F^{\prime}$ on $F_{1}^{\prime}$, $F^{\prime} \subset F, F_{1}^{\prime} \subset F_{1}, F$ and $F_{1}$ complete normed vector spaces and if $F^{\prime}$ is a closed subspace of $F$, then $T$ is continuous and consequently bounded (that is, there exists a $M>0$ with $\|T f\|_{1} \leqq M\|f\|_{)}$. The image $F_{1}^{\prime}$ is either $=F_{1}$ or of first category in $F_{1}$.

Before we apply this theorem we shall prove the following lemma:

LEMma. Let $F_{1}$ and $F_{2}$ be classes with r.k.'s and let $F_{0}$ be their intersection $F_{1} \cdot F_{2}$. The correspondence transforming $f \in F_{0}$ considered as belonging to $F_{1}$ into $f$ considered as belonging to $F_{2}$ is a closed linear transformation.

In fact, suppose that $\left\{f_{n}\right\} \subset F_{0}$ and that $f_{n}$ converges strongly to $f^{\prime}$ in $F_{1}$ and to $f^{\prime \prime}$ in $F_{2}$. Following Theorem III

$$
f^{\prime}(x)=\Phi-\lim f_{n}(x)=f^{\prime \prime}(x) .
$$

Therefore, $f^{\prime}=f^{\prime \prime} \in F_{0}$, which proves the lemma.

Theorem IV. Let $F$ and $F_{1} \subset F$ be (R.K.)-classes and $\|,\| \|_{1}$ some norms corresponding to $F$ and $F_{1}$. Then there exists a constant $M>0$ such that $\|f\| \leqq M\|f\|_{1}$ for $f \in F_{1}$.

In fact the identical transformation of $F_{1}$ considered as subspace of $F_{1}$ 
on $F_{1}$ as subspace of $F$ is closed (following the lemma), $F_{1}$ is a closed subspace of $F_{1}$, and thus our theorem follows immediately from Banach's theorem.

COROLlARY IV $I_{1}$. Let \|\| and \|\|$_{1}$ be two norms corresponding to the same (R.K.)-class $F$. There exist two positive constants $m$ and $M$ such that $m\|f\|$ $\leqq\|f\|_{1} \leqq M\|f\|$, for $f \in F$.

Theorem IV, together with Theorems I and II from $\$ 7$ and with the remark that to norm $M\|\|$ corresponds the kernel $\left(1 / M^{2}\right) K$, gives immediately the corollaries:

Corollary $\mathrm{IV}_{2}$. Let $K$ and $K_{1}$ be two p. matrices, $F$ and $F_{1}$ the corresponding classes. In order that $F_{1} \subset F$ it is necessary and sufficient that there exists a positive constant $M$ such that $K_{1} \ll M K$.

COROLlaRY IV $\mathrm{IV}_{3}$. Under the hypotheses of corollary $\mathrm{IV}_{2}$, in order that $F_{1}=F$ it is necessary and sufficient that there exist two positive constants $m$ and $M$ such that $m K \ll K_{1} \ll M K$.

The second part of Banach's theorem together with our lemma leads to the following remark which belongs to the subject matter of section (A).

REMARK. If $\left\{F_{n}\right\}$ is a strictly increasing sequence of (R.K.)-classes, then their sum $F=\sum F_{n}$ is not a (R.K.)-class. In fact, were there a norm $\|$ in $F$ giving it the structure of a Hilbert space with r.k., the subspaces $F_{n} \subset F$ would be of first category in $F$ and therefore $F$ would be of first category in itself which is impossible.

(D) Connection with existence domains in a Hilbert space. We shall now use the notion introduced recently by J. Dixmier [1] of domains of existence in a Hilbert space. A linear subset of a Hilbert space is called a domain of existence, d.e., if there exists a closed linear transformation defined in this subspace and transforming it into a subspace of another Hilbert space (which, in particular, may be identical to the first Hilbert space). The d.e.'s $D$ in a given Hilbert space $\mathfrak{S}$ with norm \|\| may be characterized by the following property: there exists a norm \|\|$_{1}$ defined in $D$ giving it the structure of a Hilbert space and satisfying

$$
\|h\|_{1} \geqq\|h\| \quad \text { for every } h \in D \text {. }
$$

In fact, if $D$ is a d.e., then we consider the linear closed transformation $T$ of $D$ into a subspace of some Hilbert space $\mathfrak{S}^{\prime}$, with the norm \|\|$^{\prime}$. It is then clear that the norm \|\|$_{1}$ defined by

$$
\|h\|_{1}^{2}=\|h\|^{2}+\|T h\|^{\prime 2}
$$

gives $D$ the character of a complete Hilbert space which satisfies condition (3). 
On the other hand, suppose that a norm \|\|$_{1}$ is defined in $D$ satisfying (3) and giving $D$ the character of a complete Hilbert space. Then the correspondence transforming any element of $D$, considered as a subspace of $\mathfrak{S}$, into the same element considered in the Hilbert space $D$ (with norm \|\|$_{1}$ ) is obviously a closed transformation and $D$ is therefore a d.e.

Using Theorem II from $\$ 7$ and Theorem IV of section (C) we prove now immediately the following theorem.

THEOREM V. If a class of functions $F$ forms a Hilbert space with a reproducing kernel, then for any linear subclass $F_{1} \subset F$, the necessary and sufficient condition in order that $F_{1}$ be a (R.K.)-class is that $F_{1}$ be a d.e. in $F$.

If we have two classes of functions $F_{1}, F_{2}$, with reproducing kernels, we can combine these two classes in different ways in order to form new classes. Let us consider in particular the following classes of functions: $F_{0}=F_{1} \cdot F_{2}$ and $F=F_{1}+F_{2}$.

THEOREM VI. If $F_{1}$ and $F_{2}$ are (R.K.)-classes, then the same is true of the classes $F_{1} \cdot F_{2}$ and $F_{1} \dot{+} F_{2}$.

Proof. The linearity of the classes is obvious. We take firstly the intersection $F_{0}$. With any norms \|\|$_{1}$ and \|\|$_{2}$ corresponding to $F_{1}$ and $F_{2}$ we define the norm in $F_{0}$ by the equation

$$
\|f\|^{2}=\|f\|_{1}^{2}+\|f\|_{2}^{2}
$$

This norm clearly defines a quadratic metric in $F_{0}$ satisfying all the required properties. For instance, the completeness of $F_{0}$ results immediately from the lemma of section (C). for $F_{0}$.

As $\|f\| \geqq\|f\|_{1}$ for $f \in F_{0}$, Theorem II, $\$ 7$ gives then the existence of a r.k.

In the case of the sum, $F=F_{1}+F_{2}$, we may apply the theorem of $\S 6$ which states that $K_{1}$ and $K_{2}$ being the r.k.'s with the norms \|\|$_{1}$ and \|\|$_{2}$ of $F_{1}$ and $F_{2}, K_{1}+K_{2}$ is the reproducing kernel of our class $F$.

Besides the operations of $\cdot$ and $\dot{+}$, we can also introduce the direct product $F_{1} \otimes F_{2}$ as defined in $\S 8$ as another operation leading to a (R.K.)-class when $F_{1}$ and $F_{2}$ are (R.K.)-classes. The class $F_{1} \otimes F_{2}$ however is defined not in $E$ but in the product set $E \times E$. If we take its restriction to the diagonal set of all pairs $\{x, x\}$, we get a class of functions defined in $E$. It can be proved that this class does not depend on the choice of norms in $F_{1}$ and $F_{2}$ as long as the norms give to $F_{1}$ and $F_{2}$ the structure of a Hilbert space with a r.k.

\section{PART II. EXAMPLES}

1. Introductory remarks. In this part we shall give examples showing how our general theory may be applied in particular cases and to what kind of results it leads. With a few exceptions we will not go into the details of calcula- 
tion and will not give in explicit form the formulas and relations obtainable by our general methods.

We shall treat essentially two kinds of kernels: the Bergman's kernels $K\left(z, z_{1}\right)$ and the harmonic kernels $H\left(z, z_{1}\right)$.

(1) Bergman's kernels. These kernels correspond to a domain $D$ in the space of $n$ complex variables $z=\left(z^{(1)}, z^{(2)}, \cdots, z^{(n)}\right)$. We consider the class $\mathfrak{A} \equiv \mathfrak{A}_{D}$ of all analytic regular functions in $D$ with a finite norm given by

$\|f\|^{2}$

$$
=\iint_{D} \cdots \iint\left|f\left(z^{(1)}, z^{(2)}, \cdots, z^{(n)}\right)\right|^{2} d x^{(1)} d y^{(1)} d x^{(2)} d y^{(2)} \cdots d x^{(n)} d y^{(n)},
$$

where $z^{(k)}=x^{(k)}+i y^{(k)}$.

The class $\mathfrak{A}$ possesses a reproducing kernel $K \equiv K_{D}$-the Bergman's kernel corresponding to $D$.

In our examples we shall consider essentially the case of plane domains $D$. If $D$ is multiply-connected we shall consider also the reduced Bergman's kernel $K^{\prime}\left(z, z_{1}\right)$, which is the reproducing kernel of the subspace $\mathfrak{A}^{\prime}$ of $\mathfrak{A}$ consisting of all functions of $\mathfrak{A}$ with a uniform integral $\int z f d z$. If $D$ is of finite connection $n$, the complementary subspace $\mathfrak{A} \Theta \mathfrak{X}^{\prime}$ is $(n-1)$-dimensional and is generated by $n$ functions $w_{n}^{\prime}(z)$ (between which there is the linear relation $\left.\sum w_{k}^{\prime}=0\right)$ defined in the following way: if $B_{k}, k=1,2, \cdots, n$, are the boundary components of the boundary $B$ of $D, w_{k}^{\prime}$ is the derivative (which is uniform) of the multiform analytic function $w_{k}$ whose real part is the harmonic measure $u_{k}$ of $D$ corresponding to $B_{k}$, that is, the harmonic function regular in $D$, equal to 1 on $B_{k}$, and vanishing on all the other components $B_{i}$.

The functions $w_{k}^{\prime}$ belong always to $\mathfrak{A}$ and are orthogonal to $\mathfrak{U}^{\prime}$. We have the relation

$$
K\left(z, z_{1}\right)=K^{\prime}\left(z, z_{1}\right)+\sum_{i, j} c_{i j} w_{i}^{\prime}(z) \overline{w_{j}^{\prime}\left(z_{1}\right)}
$$

where $\sum$ is the r.k. of $\mathfrak{A} \ominus \mathfrak{A}^{\prime}$. Consequently the matrix $\left\{c_{i j}\right\}$ is definite positive (see \$3) and it is the conjugate inverse of the Gramm's matrix $\left\{\left(w_{i}^{\prime}, w_{j}^{\prime}\right)\right\}$.

Bergman's kernels possess an important property of invariance: in case of domains in the space of $n$ variables $z^{(1)}, \cdots, z^{(u)}$, if $T$ represents $D$ pseudoconformally on $D^{\prime}$, then

$$
K_{D^{\prime}}\left(z^{\prime}, z_{1}^{\prime}\right) \partial T(z) \overline{\partial T\left(z_{1}\right)}=K_{D}\left(z, z_{1}\right) .
$$

Here, $z^{\prime}=T(z), z_{1}^{\prime}=T\left(z_{1}\right)$, and $\partial T(z)$ is the Jacobi determinant of $T$. In the case of domains in the plane, if $t(z)$ represents $D$ conformally on $D^{\prime}$, this formula takes the form

$$
K_{D^{\prime}}\left(z^{\prime}, z_{1}^{\prime}\right) \cdot t^{\prime}(z) \overline{t^{\prime}\left(z_{1}\right)}=K_{D}\left(z, z_{1}\right) .
$$


The importance of the Bergman kernels lies in the possibility they offer of generalizing different theorems on analytic functions of one complex variable to functions of several complex variables (such as Schwarz's lemma, distortion theorems, representative domains in pseudo-conformal mappings).

In the case of one variable almost all the important conformal mappings are expressible in terms of these kernels. For instance if $D$ is a simply connected domain, the mapping function $\zeta=f\left(z, z_{0}\right)$ which represents $D$ on a circle $|\zeta|<R$ in such a way that the point $z_{0} \in D$ goes into $\zeta=0$ and $f^{\prime}\left(z_{0}, z_{0}\right)=1$ is given by

$$
f\left(z, z_{0}\right)=\frac{1}{K\left(z_{0}, z_{0}\right)} \int_{z_{0}}^{z} K\left(t, z_{0}\right) d t .
$$

(2) Harmonic kernels. Consider in a plane domain $D$ (we could consider also a domain in $n$-dimensional space) the class $\mathfrak{B} \equiv \mathfrak{B}_{D}$ of all regular harmonic functions (in general complex-valued) with a finite norm given by

$$
\|h\|^{2}=\iint_{D}|h|^{2} d x d y, \quad z=x+i y .
$$

This class possesses a reproducing kernel which will be denoted by $H\left(z, z_{1}\right)$.

It should be remarked that another harmonic kernel is often considered, namely the one which corresponds to the Dirichlet metric

$$
\|h\|^{2}=\iint_{D}\left[\left|h_{x}^{\prime}\right|^{2}+\left|h_{y}^{\prime}\right|^{2}\right] d x d y .
$$

This kernel is easily expressible by Bergman's kernel and consequently does not present any additional difficulties to the ones encountered in the study and computation of Bergman's kernels.

The situation is different for the kernel $H$. Even for very simple domains (for instance for a rectangle) there is no known explicit expression of $H$ even in the form of an infinite development. (We disregard here the developments in terms of a complete non-orthogonal system which are always possible to establish for a r.k., but whose coefficients are quotients of determinants of growing orders.)

One reason for the greater difficulty of the investigation of the kernels $H\left(z, z_{1}\right)$ as compared to Bergman's kernels lies in the fact that $H$ has no such invariancy property vis-à-vis conformal transformations as have Bergman's kernels.

The interest of the kernel $H$ lies in its connection with the biharmonic problem which governs the question of equilibrium of elastic plates.

The kernel $H$ gives a simple expression for a function $u(z)$ such that $u=\partial u / \partial n=0$ on the boundary $B$ of $D$ and $\Delta \Delta u=\phi$ in $D$, for a given function $\phi$. 
Supposing that we know a function $\psi$ such that $\Delta \psi=\phi$ (we can take as $\psi$ the logarithmic potential of $\left.\phi: \psi(z)=(1 / \pi) \iint_{D} \log \left|z-z^{\prime}\right| \phi\left(z^{\prime}\right) d x^{\prime} d y^{\prime}\right)$ we get for $u$ the expression (where $g$ is the ordinary Green's function)

$$
u(z)=-\iint_{D} g\left(z, z^{\prime}\right) d x^{\prime} d y^{\prime}\left[\psi\left(z^{\prime}\right)-\iint_{D} H\left(z^{\prime}, z^{\prime \prime}\right) \psi\left(z^{\prime \prime}\right) d x^{\prime \prime} d y^{\prime \prime}\right] .
$$

The Green's function $g_{I I}\left(z, z_{1}\right)$ of the biharmonic problem, satisfying $\Delta \Delta g_{I I}=0$ for $z \neq z_{1}, g_{I I}=\partial g_{I I} / \partial n=0$ on the boundary, is given by

$$
\begin{aligned}
g_{I I}\left(z, z_{1}\right)= & \iint_{D} g\left(z, z^{\prime}\right) g\left(z^{\prime}, z_{1}\right) d x^{\prime} d y^{\prime} \\
& -\iint_{D} g\left(z, z^{\prime}\right) d x^{\prime} d y^{\prime} \iint_{D} H\left(z^{\prime}, z^{\prime \prime}\right) g\left(z^{\prime \prime}, z_{1}\right) d x^{\prime \prime} d y^{\prime \prime} .
\end{aligned}
$$

These formulas were essentially noticed already by S. Zaremba [2].

2. Comparison domains. Consider two domains in the plane, $D$ and $D^{\prime}, D \subset D^{\prime}$. The kernels $K_{D^{\prime}}$ or $H_{D^{\prime}}$, restricted to the domain $D$, are reproducing kernels of classes $\mathfrak{A}^{0}$ or $\mathfrak{B}^{0}$ formed by the restrictions of functions from $\mathfrak{A}_{D^{\prime}}$ or $\mathfrak{B}_{D^{\prime}}$. As any analytic or harmonic function vanishing in $D$ vanishes everywhere, any function $f_{0}$ of $\mathfrak{Q}^{0}$ or $\mathfrak{B}^{0}$ is a restriction of only one function $f$ from $\mathfrak{A}_{D^{\prime}}$ or $\mathfrak{B}_{D^{\prime}}$ and, following $\S 5$, Part $I$, the norm $\left\|f_{0}\right\|^{0}=\|f\|^{\prime}$. It is then clear that every $f_{0} \in \mathfrak{H}^{0}$ belongs to $\mathfrak{A}_{D}$ and that $\left\|f_{0}\right\|^{0} \geqq\left\|f_{0}\right\|$.

We can apply Theorem II of $\S 7, I$, which gives

$$
K_{D^{\prime}}^{0} \ll K_{D}, \quad K_{D^{\prime}}^{0} \text { being the restriction of } K_{D^{\prime}} \text { to } D \text {. }
$$

In the same way we get

$$
H_{D^{\prime}}^{0} \ll H_{D}
$$

If the kernel $K_{D}$ is known, we get immediately the well known estimates for the kernel $K_{D^{\prime}}$ :

$$
K_{D^{\prime}}(z, z) \leqq K_{D}(z, z),\left|K_{D^{\prime}}\left(z, z_{1}\right)\right| \leqq\left(K_{D}(z, z) K_{D}\left(z_{1}, z_{1}\right)\right)^{1 / 2}
$$

for points $z$ and $z_{1}$ belonging to $D$.

But the relation (1) (or (2)) allows much better estimates. Suppose that the kernel $K_{D^{\prime}}$ is known. For two points $z$ and $z_{1}$ in $D$ take domains $D^{\prime \prime}$ and $D_{1}^{\prime \prime}$ such that $z \in D^{\prime \prime} \subset D, z_{1} \in D_{1}^{\prime \prime} \subset D$ and that the kernels $K_{D^{\prime \prime}}$ and $K_{D_{1^{\prime}}}$ be known (for instance circles). Then, from (1), we get $K_{D}(z, z) \leqq K_{D_{1}^{\prime \prime}}(z, z)$, $K_{D}\left(z_{1}, z_{1}\right) \leqq K_{D_{1}^{\prime \prime}}\left(z_{1}, z_{1}\right)$,

$$
\begin{aligned}
\mid K_{D}\left(z, z_{1}\right)-K_{D^{\prime}}(z, & \left.z_{1}\right)\left.\right|^{2} \\
& \leqq\left[K_{D}(z, z)-K_{D^{\prime}}(z, z)\right]\left[K_{D}\left(z_{1}, z_{1}\right)-K_{D^{\prime}}\left(z_{1}, z_{1}\right)\right] \\
& \leqq\left[K_{D^{\prime \prime}}(z, z)-K_{D^{\prime}}(z, z)\right]\left[K_{D_{1}^{\prime \prime}}\left(z_{1}, z_{1}\right)-K_{D^{\prime}}\left(z_{1}, z_{1}\right)\right] .
\end{aligned}
$$


If we consider a boundary-point $t$ where the boundary has a finite curvature and if we fix $z_{1}$ and move $z$ towards $t$, the estimate (3) will grow like $1 /|z-t|$. The estimate (4) by a convenient choice of the comparison domains $D^{\prime}, D^{\prime \prime}$, and $D_{1}^{\prime \prime}$ will give a bound for $\left|K_{D}\left(z, z_{1}\right)\right|$ growing only like $1 /|z-t|^{1 / 2}$.

To show the interest of this improvement, take $D$ simply-connected and consider the conformal mapping of $D$ on a circle $|\zeta|<R$ given by

$$
\zeta=\frac{1}{K\left(z_{0}, z_{0}\right)} \int_{z_{0}}^{z} K\left(z, z_{0}\right) d z .
$$

Our problem will be to compute the point $\tau$ on the circumference $|\zeta|=R$ corresponding to $t$ on the boundary $B$. As the kernel $K$ is not known we approximate it by a development in orthogonal functions. This development may converge fairly quickly inside the domain but in general it will not converge on the boundary and will converge less and less well the nearer we come to the boundary.

To calculate $\tau$ we have to integrate from $z_{0}$ to the point $t$ on the boundary. We cannot integrate term by term the development of $K$ as it does not converge on the boundary. What we do then is to find, with the help of the estimate (4), a point $z_{1}$ near $t$ for which the integral $\int_{z_{1}}^{t}\left|K\left(z, z_{0}\right)\right| d z$ is sufficiently small. We can integrate the development of $K$ term by term from $z_{0}$ to $z_{1}$ and obtain as good an approximation of $\tau$ as we wish.

It is clear that with the estimate (3) we would not be able to do this.

3 . The difference of kernels. As we saw in $\$ 2$, in $D \subset D_{1}$, the kernels $K \equiv K_{D}$ and $K_{1} \equiv K_{D_{1}}$ satisfy the relation $K_{1} \ll K$ (the kernel $K_{1}$ being restricted to $D$ ). To illustrate the developments of $\$ 7$, I, let us investigate the class of functions $F_{2}$ corresponding to the p. matrix $K_{2}$ given by

$$
K_{2}\left(z, z_{1}\right)=K\left(z, z_{1}\right)-K_{1}\left(z, z_{1}\right) \quad z \text { and } z_{1} \text { in } D .
$$

Following the notation in the proof of Theorem II, $\S 7$, I (where $F=\mathfrak{A}$, $\left.F_{1}=\mathfrak{A}_{1}\right)$, we introduce the operator $L$ in $\mathfrak{A}$ by

$$
L f=f_{1}\left(z_{1}\right)=\left(f, K_{1}\left(z, z_{1}\right)\right)=\iint_{D} f(z) \overline{K_{1}\left(z, z_{1}\right)} d x d y .
$$

If we consider the Hilbert space $\mathfrak{S}_{1}$ of all functions $u(z)$ in square integrable in $D_{1}$ with the norm

$$
\|u\|_{1}^{2}=\iint_{D}|u(z)|^{2} d x d y
$$

the general property of r.k.'s as projections shows that $f_{1}(z)$, as function in $D_{1}$, is the projection on $\mathfrak{A}_{1}$ of the function $\tilde{f}(z)=f(z)$ in $D$ and $=0$ in $D_{1}-D$. Consequently,

$$
\left\|f_{1}(z)\right\| \leqq\left\|f_{1}(z)\right\|_{1} \leqq\|\tilde{f}(z)\|_{1}=\|f(z)\|
$$


The second inequality may become equality for $f(z) \neq 0$ only in the case when $D_{1}-D$ is of two-dimensional measure 0 (for instance when $D$ differs from $D_{1}$ only by some slits). We will exclude this case and consequently

$$
\left\|f_{1}(z)\right\|=\|L f\|<\|f\| \quad \text { for } f \neq 0 .
$$

We introduce then the operator $L^{\prime}$ by

$$
L^{\prime 2}=I-L \text {. }
$$

The subspace $F_{0}$ is here reduced to 0 as $0 \neq f=L f$ is impossible in view of (3). Therefore $F^{\prime}=F \equiv \mathfrak{A}$ and the only possibilities for the class $F_{2}$ are: $1^{\circ}$, $F_{2}=\mathfrak{A}$ or $2^{\circ}, F_{2}$ is a dense subspace of $\mathfrak{A}$.

The first case represents itself always when $D$ is completely interior to $D_{1}\left(\overleftarrow{D} \subset D_{1}\right)$. In fact, the operator $L$ is then completely continuous. To prove this we take a sequence $\left\{g^{(n)}\right\} \subset \mathfrak{A}$ converging weakly to $g \in \mathfrak{A}$. The functions $\tilde{g}^{(n)}$ converge then weakly in $\mathfrak{S}_{1}$ to $\tilde{g}$ and their projections $g_{1}^{(n)}$ on $\mathfrak{A}_{1}$ converge weakly to $g_{1}$. But the weak convergence in $\mathfrak{A}_{1}$ involves uniform convergence of $g_{1}^{(n)}(z)$ towards $g_{1}(z)$ in any closed subset of $D_{1}$, in particular in $\bar{D}$ (see section $(5), \S 2, I)$. When we restrict the functions $g_{1}^{(n)}(z)$ and $g_{1}(z)$ to $D$ they become the transforms $L g^{(n)}$ and $L g$. Therefore, the uniform convergence of $g_{1}^{(n)}$ to $g_{1}$ in $\bar{D}$ involves the strong convergence of $L g^{(n)}$ to $L g$ in the space $\mathfrak{A}$.

Following our remarks after Theorem III, §7, I, the class $F_{2}=F^{\prime}=\mathfrak{A}$. To get the norm $\|f\|_{2}$ corresponding to the kernel $K_{2}$, we have to find the solution $g(z)$ of the equation

$$
g-L g=f
$$

which exists and is unique for every $f \in \mathfrak{A}$. Then

$$
\|f\|_{2}^{2}=\|g\|^{2}-\left\|g_{1}\right\|_{1}^{2}
$$

where

$$
g_{1}\left(z_{1}\right)=L g=\iint_{D} g(z) K_{1}\left(z, z_{1}\right) d x d y .
$$

Let us note that the operator $L$ which in general has a bound not greater than 1 has here a bound less than 1 .

To illustrate the second case we have to take a domain having common boundary points with the boundary of $D_{1}$. It seems probable that for every such domain $D$ we shall be in the second case (at least if one of the boundary components of $D$ arrives at the boundary of $D_{1}$ ).

To prove that we are in the second case, we have to show that the operator $L$ has a bound $=1$. Then $L$ cannot be completely continuous (as the bound is not attained). The class $F_{1}$ is a proper subclass of $\mathfrak{A}$. The class $F_{2}^{\prime}$ of all functions 


$$
f_{2}(z)=g(z)-g_{1}(z), \quad \text { where } g_{1}=L g, g \in \mathfrak{A},
$$

is a proper subspace of $F_{2}$, dense in $F_{2}$. For such a function $f_{2}$, the norm in $F_{2}$ is given by

$$
\left\|f_{2}\right\|_{2}^{2}=\|g\|^{2}-\left\|g_{1}\right\|_{1}^{2}
$$

The class $F_{2}$ in the metric of $\mathfrak{A}$ is a dense subspace of $\mathfrak{A}$. There are functions $f \in \mathfrak{A}$ which do not belong to $F_{2}$ and for which, a fortiori, the equation $f=g-L g$ has no solution $g \in \mathfrak{A}$. (There may be such a solution, analytic in $D$ but not in square integrable in $D$.)

For two explicitly given domains $D \subset D_{1}$, it may not be easy to prove that we are in the second case by using the property that the bound of $L$ is 1 . We can transform this property into another one, more easily proved.

To this effect we shall consider for any function $f_{1} \in \mathfrak{A}_{1}$ the quotient

$$
Q\left(f_{1}\right)=\left\|f_{1}\right\|^{2} /\left\|f_{1}\right\|_{1}^{2} \text {. }
$$

LEMMA. In order that the bound of $L$ be 1 it is necessary and sufficient that there exist functions $f_{1}$ with $Q\left(f_{1}\right)$ as near 1 as we wish.

To prove this lemma we remark firstly that the l.u.b. $Q\left(f_{1}\right)$ for $f_{1} \in \mathfrak{A}_{1}$ is the same as the l.u.b. $Q(L f)$ for $f \in \mathfrak{A}$. In fact the $L f$ form a dense subspace in the space $\mathfrak{A}_{1}$ (otherwise there would be a $g_{1} \in \mathfrak{A}_{1}, g_{1} \neq 0$, with $\left(g_{1}, L f\right)_{1}=0$ for every $f \in \mathfrak{A}$. Therefore $\left(g_{1}, f\right)=\left(g_{1}, L f\right)_{1}=0$ and $g_{1}=0$ in $D$ which involves $g_{1}=0$ in $\left.D_{1}\right)$. Consequently, there is for every $f_{1} \in \mathfrak{A}_{1}$ a function $f \in \mathfrak{A}$ with $\left\|f_{1}-L f\right\|_{1}$ as small as we wish. As $\left\|f_{1}-L f\right\| \leqq\left\|f_{1}-L f\right\|_{1}$ it is clear that $Q(L f)$ will approach $Q\left(f_{1}\right)$ as nearly as we wish.

Now, $Q(L f)$ can be represented as

$$
Q(L f)=\frac{(L f, L f)}{(L f, L f)_{1}}=\frac{(L f, L f)}{(L f, f)} .
$$

Our lemma amounts to the equivalence of the two properties, for any $\alpha$, $0<\alpha \leqq 1$ :

$$
\begin{aligned}
& (L f, L f) \leqq \alpha^{2}(f, f) \\
& (L f, L f) \leqq \alpha(L f, f)
\end{aligned}
$$$$
\text { for all } f \in \mathfrak{A} \text {, }
$$$$
\text { for all } f \in \mathfrak{A} \text {. }
$$

From $\left(6^{\prime \prime}\right)$ follows $\left(6^{\prime}\right)$ :

$$
\begin{gathered}
(L f, L f) \leqq \alpha(L f, f) \leqq \alpha(L f, L f)^{1 / 2}(f, f)^{1 / 2} \\
(L f, L f)^{1 / 2} \leqq \alpha(f, f)^{1 / 2}, \quad(L f, L f) \leqq \alpha^{2}(f, f) .
\end{gathered}
$$

From (6') follows

$$
(L f, f) \leqq \alpha(f, f)
$$


In fact, we have

$$
(L f, f) \leqq(L f, L f)^{1 / 2}(f, f)^{1 / 2} \leqq \alpha(f, f)^{1 / 2}(f, f)^{1 / 2}=\alpha(f, f) .
$$

From $\left(6^{\prime \prime \prime}\right)$ follows $\left(6^{\prime \prime}\right)$ : we use the fact that $L$ is positive which gives $(L f, g) \leqq(L f, f)^{1 / 2}(L g, g)^{1 / 2}$. Then

$$
\begin{aligned}
(L f, L f) & \leqq(L f, f)^{1 / 2}(L L f, L f)^{1 / 2} \leqq(L f, f)^{1 / 2} \alpha^{1 / 2}(L f, L f)^{1 / 2}, \\
(L f, L f)^{1 / 2} & \leqq \alpha^{1 / 2}(L f, f)^{1 / 2}, \quad(L f, L f) \leqq \alpha(L f, f) .
\end{aligned}
$$

This proves our lemma.

We shall apply the lemma to two domains $D \subset D_{1}$ having a common boundary point which belongs also to the boundary of the exterior of $D$. We suppose further that at this boundary point the boundaries of $D$ and $D_{1}$ have a common tangent. We can take the common boundary point as the origin $O$ and the inner normal of the boundaries at this point as the positive axis.

It is then easily verified that the functions

$$
f_{n}(z)=\frac{1}{(n z+1)^{2}}, \quad n=1,2, \cdots,
$$

for sufficiently great values of $n$, belong to $\mathfrak{A}_{1}$ and that they satisfy the asymptotic equation

$$
\left\|f_{n}\right\| \sim\left\|f_{n}\right\|_{1} \quad \text { for } n \rightarrow \infty .
$$

This shows that the l.u.b. $Q(f)=1$ and that we are in the second case.

Let us consider now another kind of example which we excluded till now. Namely, we shall suppose that the domain $D$ differs from $D_{1}$ only by a number of slits of finite length. It is then immediately seen that for a function $f_{1} \in \mathfrak{A}_{1}$, considered as belonging to $\mathfrak{A}_{1}^{0} \subset \mathfrak{A}\left(\mathfrak{H}_{1}^{0}=\right.$ class $\mathfrak{A}_{1}$ restricted to $\left.D\right)$, we have

$$
\left\|f_{1}\right\|=\left\|f_{1}\right\|_{1} .
$$

Consequently $\mathfrak{A}_{1}^{0}$ is a closed linear subspace of $\mathfrak{A}$ and the kernel $K\left(z, z_{1}\right)$ $-K_{1}\left(z, z_{1}\right)$ corresponds to the subspace $\mathfrak{A} \Theta \mathfrak{U}_{1}^{0}$.

4. The square of a kernel introduced by Szegö. We shall now give an application of Theorem II, $\S 8$, I. Consider a domain in the plane with a sufficiently smooth boundary (for simplicity's sake we may suppose that the boundary curves are analytic). For this domain we shall consider a kernel, first introduced by Szegö [1], which we shall denote by $k\left(z, z_{1}\right)$. This kernel corresponds to the class $S$ of all analytic functions which possess in square integrable boundary values. As the functions are not necessarily continuous on the boundary we have to specify the meaning of the boundary values of the functions. We shall suppose that for such a function $f(z)$, its integral $F(z)$ is a continuous function in the closed domain (but $F(z)$ may be a multi- 
form function). Then we suppose that for any two points $t_{1}, t_{2}$ of the same boundary curve the difference $F\left(t_{2}\right)-F\left(t_{1}\right)$ may be represented by the integral

$$
\int_{t_{1}}^{t_{2}} f(t) d t
$$

where $f(t)$ is defined almost everywhere on the boundary curves and is in square integrable there. The integral is taken over an arc of the boundary curve going from $t_{1}$ to $t_{2}$.

The function $f(t)$ will be considered as the boundary value of $f(z)$ in the boundary-point $t . f(t)$ is completely determined by $f(z)$ with exception of a boundary set of linear measure zero. The existence of boundary values of $f(z)$ in our sense is equivalent to the absolute continuity of $F(z)$ on the boundary. With the boundary values taken in this sense we define a norm in class $S$ by the equation

$$
\|f\|^{2}=\sum \int_{C_{\nu}}|f(t)|^{2} d s,
$$

where $C_{\nu}$ are the boundary curves and $d s$ is the element of length on the curve. For the functions of class $S$ it is easily proved that the Cauchy theorem is still valid in the form

$$
f(z)=\sum \int_{C_{\nu}} \frac{f(t)}{t-z} d t .
$$

From this it is immediately seen that the class possesses a reproducing kernel which is the kernel $k\left(z, z_{1}\right)$ introduced by Szegö. Quite recently, in his thesis, P. Garabedian [1] proved that

$$
k^{2}\left(z, z_{1}\right)=\frac{1}{4 \pi} K\left(z, z_{1}\right)+\sum_{i, j} \alpha_{i, j} w_{i}^{\prime}(z) w_{j}^{\prime}\left(z_{1}\right)
$$

where $K$ is the Bergman kernel for the domain and $w_{i}^{\prime}$ are the functions introduced in $\$ 1$.

In cases of simply-connected domains Garabedian's formula takes a very simple form, namely:

$$
k^{2}\left(z, z_{1}\right)=\frac{1}{4 \pi} K\left(z, z_{1}\right) .
$$

In this case Theorem II, §8, I, gives a property of analytic functions which seems to have been unnoticed even for this simple case. Every function $f(z)$ in square integrable in the domain $D$ is representable in infinitely many ways by a series

$$
f(z)=\sum \phi_{k}(z) \psi_{k}(z)
$$


where the functions $\phi_{k}(z)$ and $\psi_{k}(z)$ are in square integrable on the boundary. In addition, we have the formula

$$
4 \pi \iint_{D}|f(z)|^{2} d x d y=\min \sum_{k} \sum_{l} \int_{C} \phi_{k}(t) \overline{\phi_{l}(t)} d s \int_{C} \psi_{k}(t) \overline{\psi_{l}(t)} d s,
$$

the minimum being extended to all representations of $f(z)$ in the form (2). In particular, we have the inequality

$$
4 \pi \iint_{D}|\phi(z) \psi(z)|^{2} d x d y \leqq \int_{C}|\phi(t)|^{2} d s \int_{C}|\psi(t)|^{2} d s .
$$

In the case of a multiply-connected domain the problem is a little more complicated because of the presence of the functions $w_{k}^{\prime}$. It can be proved then that if we replace the Bergman's kernel $K$ by the reduced kernel $K^{\prime}$ (see \$1) we have again a formula similar to (1):

$$
k^{2}\left(z, z_{1}\right)=\frac{1}{4 \pi} K^{\prime}\left(z, z_{1}\right)+\sum_{i, j} \beta_{i, j} w_{i}^{\prime}(z) \overline{w_{j}^{\prime}\left(z_{1}\right)}
$$

where, now, the $\beta_{i, j}$ are the coefficients of a positive quadratic hermitian form, which means that $\sum_{i, j}$ represents a positive matrix. Consequently, the functions of the class corresponding to the kernel $k^{2}$ are sums of functions in square integrable in $D$ with a uniform integral (which form the class belonging to $K^{\prime}$ ) and of a linear combination of the $w_{k}^{\prime}$ (which form the class corresponding to $\left.\sum_{i, j}\right)$. The functions $w_{k}^{\prime}$ are in square integrable in $D$, and thus every function belonging to the class of $k^{2}$ is in square integrable in $D$. Conversely, it can be proved that every function in square integrable over $D$ belongs to the class of $k^{2}$. By Theorem II, $\S 8$, I, we know that the functions belonging to $k^{2}$ are of the form (2), but we will not be able to obtain a formula like (3) for the case of a simply-connected domain. However, a more complicated formula generalizing (3) does exist. In the present case of a multiplyconnected domain, we have still the property that the product $\phi(z) \psi(z)$ is in square integrable in $D$ if $\phi$ and $\psi$ are in square integrable on the boundary, but the inequality between the integrals will not be as simple as in the case of a simply-connected domain.

5. The kernel $H\left(z, z_{1}\right)$ for an ellipse. We shall construct the kernel $H\left(z, z_{1}\right)$ for the ellipse $D$

$$
D: \frac{x^{2}}{a^{2}}+\frac{y^{2}}{b^{2}}=1, \quad a>b,
$$

by use of an orthonormal complete system( $\left.{ }^{11}\right)$.

(11) The system and the corresponding expression for the kernel were communicated to us by A. Erdélyi. It should be noted that the system was already introduced by S. Zaremba [1] who also noticed that it is orthogonal and complete in the Dirichlet metric. 
We write

$$
\begin{aligned}
a & =h \cosh \epsilon, & b & =h \sinh \epsilon, \\
z=x+i y & =h \cosh \zeta, & \zeta & =\xi+i \eta .
\end{aligned}
$$

(3) gives us a conformal transformation of the rectangle $R, 0<\xi<\epsilon$, $-\pi<\eta<\pi$ on the ellipse (1) with the rectilinear slit $-a<x<h$. Consider in the rectangle $R$ the analytic function $p_{n}(z)=\sinh n \zeta / \sinh \zeta$ (positive integer $n)$. It is immediately seen that in the variable $z$ the function is a polynomial of degree $n-1$. Consequently, all the polynomials in the variable $z$ can be expressed as finite linear combinations of these polynomials for $n$ $=1,2,3, \cdots$. Since the harmonic polynomials form a complete system in our class $\mathfrak{B}$ of harmonic functions, the real and imaginary parts of $p_{n}(z)$ also form a complete system. On the other hand, it is easy to verify that these real and imaginary parts form an orthogonal system. This verification is made in an easy way by performing the integration in the rectangle $R$ instead of the domain $D$. Using the formula

$$
\iint_{D} f(x, y) d x d y=h^{2} \int_{0}^{\epsilon} \int_{-\pi}^{+\pi} f(x, y)|\sinh \zeta|^{2} d \xi d \eta
$$

one verifies easily that the sequence $\phi_{n}(z)$ defined by

$$
\begin{array}{ll}
\phi_{2 n-2}=\frac{2}{h}\left(\frac{n}{\pi}\right)^{1 / 2}(\sinh 2 n \epsilon+n \sinh 2 \epsilon)^{-1 / 2} \operatorname{Re} p_{n}(z), & n=1,2, \cdots, \\
\phi_{2 n-3}=\frac{2}{h}\left(\frac{n}{\pi}\right)^{1 / 2}(\sinh 2 n \epsilon-n \sinh 2 \epsilon)^{-1 / 2} \operatorname{Im} p_{n}(z), \quad n=2,3, \cdots,
\end{array}
$$

is orthonormal. We can then write the kernel of our class in the form

$$
H\left(z, z_{1}\right)=\frac{4}{h^{2} \pi} \sum_{n=1}^{\infty} n\left\{\frac{\operatorname{Re} p_{n}(z) \operatorname{Re} p_{n}\left(z_{1}\right)}{\sinh 2 n \epsilon+n \sinh 2 \epsilon}+\frac{\operatorname{Im} p_{n}(z) \operatorname{Im} p_{n}\left(z_{1}\right)}{\sinh 2 n \epsilon-n \sinh 2 \epsilon}\right\} .
$$

6. Construction of $H\left(z, z_{1}\right)$ for a strip. Our next example will use the theorem of the limit of kernels for decreasing sequences of classes (see Theorem I, $\S 9$, I). It will be at the same time an example of a representation of a kernel by use of a resolution of identity in a Hilbert space (see $\$ 10, I$ ). Consider the kernel of $\$ 1$ and suppose that in the ellipse

$$
\frac{x^{2}}{a^{2}}+\frac{y^{2}}{b^{2}}=1
$$

the axis $b<a$ remains fixed, while $a \rightarrow \infty$. The ellipse in the limit will become the horizontal strip $|y|<b$. It is clear that our theorem on the limit of kernels applies in this case and we get the kernel $H\left(z, z_{1}\right)$ for the strip as a limit of the kernels corresponding to ellipses. Before performing this passage 
to the limit, we shall at first make a few preliminary remarks.

As in the preceding paragraph, we consider the quantities $h$ and $\epsilon$ given by $b=h \sinh \epsilon, a=h \cosh \epsilon, b$ being fixed and $a \rightarrow \infty$. We see immediately that $h \rightarrow \infty$ and $\epsilon \rightarrow 0$ in such a way that $h \epsilon \rightarrow b$.

In the conformal mapping, $z=h \cosh \zeta$ we introduce a new variable $\zeta^{\prime}$ by the equation

$$
\epsilon \zeta^{\prime}=\zeta-\frac{\pi}{2} i
$$

Then $z=h \sin i \epsilon \xi^{\prime}$, and the ellipse with the slit along the real axis going from $+h$ to $-a$ is transformed in the rectangle

$$
R_{\epsilon}, \quad 0<\xi^{\prime}<1, \quad-\frac{3 \pi}{2 \epsilon}<\eta^{\prime}<\frac{\pi}{2 \epsilon} .
$$

Consider a point $z$ in the horizontal strip $|y|<b$. For sufficiently large values of $h$ the ellipse will contain $z$. Suppose that $\operatorname{Im} z>0$, then the corresponding $\zeta^{\prime}$ will lie in the upper half of the rectangle $R_{\epsilon}$ and for $h \rightarrow \infty$, $\zeta^{\prime} \rightarrow z / b i$. The point $\zeta^{\prime \prime}=\bar{\zeta}^{\prime}-\pi i / \epsilon$ will then correspond to the conjugate point $\bar{z}$, so that $\left(\zeta^{\prime \prime}+\pi i / \epsilon\right) \rightarrow \bar{z} i / b$. If we now return to the formula for the kernel from $\$ 5$ and replace the function $p_{n}(z)$ by the expression $\sinh n \zeta / \sinh \zeta$, then replace $\zeta$ by $\epsilon \zeta^{\prime}+\pi i / 2$ and separate the series which expresses the kernel $H$ (see $(5), \S 5$ ) into parts corresponding to even and odd indices, we can write the kernel in the form of a sum of four series:

$$
\begin{aligned}
\frac{4}{\pi h} \sum_{n \text { odd }} \frac{n}{h} \frac{\operatorname{Re} \frac{\cos i n \epsilon \zeta^{\prime}}{\cos i \epsilon \zeta^{\prime}} \operatorname{Re} \frac{\cos i n \epsilon \zeta_{1}^{\prime}}{\cos i \epsilon \zeta_{1}^{\prime}}}{\sinh 2 n \epsilon+n \sinh 2 \epsilon} \\
+\frac{4}{\pi h} \sum_{n \text { odd }} \frac{n}{h} \frac{\operatorname{Im} \frac{\cos i n \epsilon \zeta^{\prime}}{\cos i \epsilon \zeta^{\prime}} \operatorname{Im} \frac{\cos i n \epsilon \zeta_{1}^{\prime}}{\cos i \epsilon \zeta_{1}^{\prime}}}{\sinh 2 n \epsilon-n \sinh 2 \epsilon} \\
+\frac{4}{\pi h} \sum_{n \text { even }} \frac{n}{h} \frac{\operatorname{Re} \frac{\sin i n \epsilon \zeta^{\prime}}{\cos i \epsilon \zeta^{\prime}} \operatorname{Re} \frac{\sin i n \epsilon \zeta_{1}^{\prime}}{\cos i \epsilon \zeta_{1}^{\prime}}}{\sinh 2 n \epsilon+n \sinh 2 \epsilon} \\
+\frac{4}{\pi h} \sum_{n \text { even }} \frac{n}{\sin i n \epsilon \zeta^{\prime}} \frac{\sin i n \epsilon \zeta_{1}^{\prime}}{\cos i \epsilon \zeta^{\prime}} \operatorname{Im} \frac{\sinh 2 n \epsilon-n \sinh 2 \epsilon}{\cos i \epsilon \zeta_{1}^{\prime}}
\end{aligned}
$$

For $h \rightarrow \infty$, if we denote $n / h$ by $t$ and if we then notice the asymptotic formulas $h \epsilon \sim b, n \epsilon \sim b t, n \sin 2 \epsilon \sim 2 b t$, it is immediately seen that the series represent approximating Riemannian sums for the integrals 


$$
\begin{aligned}
& \frac{2}{\pi} \int_{0}^{\infty} t \frac{\operatorname{Re} \cos i b t \zeta^{\prime} \operatorname{Re} \cos i b t \zeta_{1}^{\prime}}{\sinh 2 b t+2 b t} d t+\frac{2}{\pi} \int_{0}^{\infty} t \frac{\operatorname{Im} \cos i b t \zeta^{\prime} \operatorname{Im} \cos i b t \zeta_{1}^{\prime}}{\sinh 2 b t-2 b t} d t, \\
& +\frac{2}{\pi} \int_{0}^{\infty} t \frac{\operatorname{Re} \sin i b t \zeta^{\prime} \operatorname{Re} \sin i b t \zeta_{1}^{\prime}}{\sinh 2 b t+2 b t} d t+\frac{2}{\pi} \int_{0}^{\infty} t \frac{\operatorname{Im} \sin i b t \zeta^{\prime} \operatorname{Im} \sin i b t \zeta_{1}^{\prime}}{\sinh 2 b t-2 b t} d t,
\end{aligned}
$$

when we subdivide the infinite interval $(0,+\infty)$ into equal intervals of length $2 / h$ and in each interval take the value of the integrated function in the center (for the first two series) or in the right end (for the two last series).

The convergence of these sums towards the integrals for $h \rightarrow \infty$ is easily verified and in this way we obtain for the kernel of the horizontal strip the expression given by the above four integrals where we replace $\zeta^{\prime}$ and $\zeta_{1}^{\prime}$ by the values $z / b i$ and $z_{1} / b i$ :

$$
\begin{aligned}
H\left(z, z_{1}\right)= & \frac{2}{\pi} \int_{0}^{\infty} \frac{\operatorname{Re} \cos z t \operatorname{Re} \cos z_{1} t+\operatorname{Re} \sin z t \operatorname{Re} \sin z_{1} t}{\sinh 2 b t+2 b t} t d t \\
& +\frac{2}{\pi} \int_{0}^{\infty} \frac{\operatorname{Im} \cos z t \operatorname{Im} \cos z_{1} t+\operatorname{Im} \sin z t \operatorname{Im} \sin z_{1} t}{\sinh 2 b t-2 b t} t d t .
\end{aligned}
$$

This integral representation of the kernel corresponds to a resolution of identity in the Hilbert space $\mathfrak{B}$ corresponding to the strip. This resolution of identity has a quadruple spectrum (see $\$ 10, I$ ) defined by the following four functions $f_{k}(z, \lambda): f_{k}(z, \lambda)=0$ for $\lambda \leqq 0$, for $\lambda>0$

$$
\begin{array}{ll}
f_{1}(z, \lambda)=\int_{0}^{\lambda} \operatorname{Re} \cos z t d t=\operatorname{Re} \frac{\sin z \lambda}{z}, & f_{2}(z, \lambda)=\operatorname{Re} \frac{1-\cos z \lambda}{z}, \\
f_{3}(z, \lambda)=\operatorname{Im} \frac{\sin z \lambda}{z}, & f_{4}(z, \lambda)=\operatorname{Im} \frac{1-\cos z \lambda}{z} .
\end{array}
$$

It is easily verified that these functions satisfy the conditions (a) and (b) from $\$ 10$, I. The condition $\left(c^{\prime}\right)$ results from the fact that the functions $f_{k}$ determine the r.k. $H\left(z, z_{1}\right)$ of the class $\mathfrak{B}$ by the formula above.

7. Limits of increasing sequences of kernels. In the preceding section we had an example of a limit of decreasing sequence of classes and kernels. We shall give here an example of an increasing sequence of kernels.

Consider the Bergman's kernels $K_{n}$ for a decreasing sequence of simplyconnected domains $D_{n}$ such that $\bar{D}_{n+1} \subset D_{n}$ and $E=\lim D_{n}=D_{1} \cdot D_{2} \cdots$ consists of the two closed circles $\bar{C}_{1}:|z-2| \leqq 1$, and $\bar{C}_{2}:|z+2| \leqq 1$, with the segment of the real axis $I:-1 \leqq x \leqq 1, y=0$.

Following the general theory of $\$ 9$, I, we have to consider the set $E_{0}$ where $K_{0}(z, z)=\lim K_{n}(z, z)<\infty$.

Every point of the open circle $C_{1}$ belongs to $E_{0}$. In fact, if $K_{(1)}$ is the Bergman's kernel for $C_{1}$, by the method of comparison domains (see \$2) we get 
$K_{n} \ll K_{(1)}$ in $C_{1}$ and consequently $K(z, z)=\lim K_{n}(z, z) \leqq K_{(1)}(z, z)$ for $z$ in $C_{1} \cdot$ The same is true for $z \in C_{2}$. We are going to prove that

$$
E_{0}=C_{1}+C_{2} \text {. }
$$

We have to show that for $z_{0} \in E-E_{0}, \lim K_{n}\left(z_{0}, z_{0}\right)=\infty$. We use again a domain of comparison. We take a closed line $L$ defining an exterior domain $S$ containing $E$ and such that by a convenient translation we can approach $L$ as near as we wish to $z_{0}$ without touching $E$. The domain $S$ will contain all $D_{n}$ from some $n$ onwards. For these $n, K_{n} \gg K_{S}$, therefore $\lim K_{n}\left(z_{0}, z_{0}\right)$ $\geqq K_{S}\left(z_{0}, z_{0}\right)$. The translation of the line $L$ (and the domain $S$ ) will have on $K_{S}\left(z_{0}, z_{0}\right)$ the effect as if the domain $S$ were fixed and the point $z_{0}$ were moving towards the boundary $L$. As for Bergman's kernels of domains of finite connection we have the theorem that $K(z, z)$ goes uniformly to $+\infty$ when $z$ approaches the boundary $\left({ }^{12}\right)$, we arrive at the result, $\lim K_{n}\left(z_{0}, z_{0}\right)=+\infty$.

For $z_{0} \in E-E_{0}$, with exception of $z_{0}= \pm 1$, we can take as $L$ a sufficiently small circumference. For $z_{0}= \pm 1$, a circumference cannot do. We take then for $L$ the boundary of a square, for instance, for $z_{0}=+1$, we take the square: $-\epsilon<x<1-\epsilon, \epsilon<y<1+\epsilon$.

Using the notation of $\$ 9, I,(B)$, we see immediately that the class $F_{0}$ with the norm \|\|$_{0}$ is here a subspace of the class $F$ of all functions $f(z)$ defined in the two open circles $C_{1}$ and $C_{2}$, analytic and regular in each (but $f(z)$ in $C_{1}$ is not necessarily an analytic continuation of $f(z)$ in $C_{2}$ ), with a finite norm

$$
\|f\|^{2}=\iint_{C_{1}}|f(z)|^{2} d x d y+\int_{C_{2}}|f(z)|^{2} d x d y .
$$

Consequently, the condition $2^{\circ}$ of $\$ 4$, I is satisfied and we obtain a functional completion $F_{0}^{*}$ of $F_{0}$. Then, it is easily proved, by using general approximation theorems of analytic functions, that the space $F_{0}^{*}$ coincides with $F$.

The r.k. of $F$ is immediately seen to be given by:

$$
\begin{aligned}
& K\left(z, z_{1}\right)=0 \text { if } z \text { and } z_{1} \text { belong to different circles, } \\
& K\left(z, z_{1}\right)=K_{(i)}\left(z, z_{1}\right) \text { if } z \text { and } z_{1} \text { belong to } C_{i} .
\end{aligned}
$$

Similar results can be obtained if the domains $D_{n}, \bar{D}_{n+1} \subset D_{n}$ have an arbitrary intersection $E=D_{1} \cdot D_{2} \cdots$. The set $E_{0}$ is then the set of all interior points of $E$.

8. Construction of reproducing kernels by the projection-formula of $\S 12$,

(12) This theorem is proved by using conformal mapping and the invariancy property of Bergman's kernels. For the kernels $H$ for which the invariancy property is not true, a similar theorem has been proved only for domains with sufficiently smooth boundaries. 
I. The formula of $\$ 12$, giving the r.k. of a sum of two closed subspaces, may serve in many cases for the construction of kernels. We shall indicate here a few cases when it can be applied.

(1) The expression for $H\left(z, z_{1}\right)$ in terms of $K\left(z, z_{1}\right)$. Consider for a domain $D$ in the plane the classes $\mathfrak{A}$ and $\mathfrak{B}$. In spite of the similarity of the metrics in the two classes, the relationship between their kernels $K$ and $H$ does not seem a simple one. We shall get such a relation by using the formula (18), $\$ 12, \mathrm{I}$.

To this effect we remark firstly that the class $\mathfrak{B}$ is a class of complex valued functions, that is, every function $h$ of the class is representable in the form $h=h_{1}+i h_{2}, h_{1}$ and $h_{2}$ harmonic and real-valued. Consequently, the class $\mathfrak{A}$ is a linear closed subspace of the class $\mathfrak{B}$. Also the class $\overline{\mathfrak{A}}$ of all antianalytic functions (that is, conjugate $\overline{f(z)}$ of analytic functions $f$ ) with a similar norm is a closed linear subspace of $\mathfrak{S}$. On the other hand, it is clear that every function $h \in \mathfrak{S}$ is representable as a sum

$$
h(z)=f_{1}(z)+\overline{f_{2}(z)}
$$

with two analytic functions $f_{1}$ and $f_{2}$. These functions are uniquely determined with the exception of additive constants. In general, in this decomposition, the functions $f_{1}$ and $\bar{f}_{2}$ may be of infinite norm, that is, they may not belong to our classes $\mathfrak{A}$ and $\overline{\mathfrak{A}}$. But when the boundary of $D$ is not too irregular it may be proved that a dense subclass of $\mathfrak{B}$ is decomposable in this form with $f_{1}$ and $\bar{f}_{2}$ belonging to $\mathfrak{A}$ and $\overline{\mathfrak{A}}$ which means that $\mathfrak{B}=\mathfrak{A} \oplus \overline{\mathfrak{A}}$. In order to avoid the indetermination of the above decomposition of $h \in \mathfrak{B}$ into $f_{1}+\bar{f}_{2}$ because of the additive constant, we shall admit to the class $\overline{\mathfrak{A}}$ only functions $\bar{f}$ satisfying the condition

$$
\iint_{D} \bar{f} d x d y=0
$$

With $\overline{\mathfrak{A}}$ so fixed, the condition $\mathfrak{A} \cdot \overline{\mathfrak{A}}=(0)$ is satisfied. Consequently, we can apply our formula to calculate the kernel $H$.

We obtain for $H$ a development of the form

$$
H\left(z, z_{1}\right)=\sum_{n=1}^{\infty}\left(\Lambda_{n-1}^{\prime}\left(z, z_{1}\right)+\Lambda_{n-1}^{\prime \prime}\left(z, z_{1}\right)-\Lambda_{n}\left(z, z_{1}\right)-\overline{\Lambda_{n}\left(z_{1}, z\right)}\right)
$$

where the $\Lambda$ 's are expressible in terms of the r.k.'s of $\mathfrak{A}$ and $\overline{\mathfrak{A}}$ following the formulas from $\$ 12$. Since the kernel for $\mathfrak{A}$ is $K\left(z, z_{1}\right)$, the kernel for $\overline{\mathfrak{A}}$ is immediately seen to be $\overline{K\left(z, z_{1}\right)}+$ const. The constant is easy to determine and we get for the kernel $\bar{K}$ of $\overline{\mathfrak{A}}$ the expression $\bar{K}\left(z, z_{1}\right)=K\left(z_{1}, z\right)-1 / \sigma$ where $\sigma$ is the area of $D$. All the terms of the development of $H$ are then expressible by repeated integration in terms of the kernel $K$ alone.

This development can serve to compute $H$ when $K$ is known. It will be especially useful when the domain is such that the subspaces $\mathfrak{A}$ and $\overline{\mathfrak{A}}$ of $\mathfrak{B}$ 
form a positive minimal angle. This is equivalent to the fact that there exists a constant $m>0$ such that

$$
m\|f\| \leqq\left\|f+\overline{f_{1}}\right\| \quad \text { for any } f \in \mathfrak{A}, \overline{f_{1}} \in \overline{\mathfrak{A}} .
$$

It is easy to see that the last condition is equivalent to the following property of harmonic, real-valued functions $h \in \mathfrak{B}$ :

$$
\|\tilde{h}\| \leqq c\|h\|, \quad \text { for some constant } c>0,
$$

where $\tilde{h}$ is the conjugate harmonic function of $h$ (that is, $h+i \tilde{h}$ is analytic). This property can be proved for domains with a fairly smooth boundary (continuous tangent), with at most a finite number of angular points with positive angles (see K. Friedrichs, [1]). Consequently we can apply (1) to compute the kernel $H$ for a rectangle, which computation would be especially useful in view of applications to rectangular elastic plates.

(2) We shall describe now a manner of applying our formula to calculate the kernels $K$.

Let $C$ be a closed set in the plane (not necessarily bounded) of twodimensional measure 0 . Usually $C$ will be composed of a finite number of arcs or closed curves. $C$ decomposes the plane in a certain number of domains $D_{1}, D_{2}, \cdots$ which together form an open set $D$. In $D$ we consider the class of functions $\mathfrak{A}_{D}$ formed by all functions which in each of the domains $D_{n}$ are analytic and regular. We call such functions locally analytic and the set $C$ their singular set $\left({ }^{13}\right)$. by

We shall suppose further that the functions of $\mathfrak{A}_{D}$ have a finite norm given

$$
\|f\|^{2}=\iint_{D}|f(z)|^{2} d x d y
$$

The class $\mathfrak{A}_{D}$ possesses a r.k. $K_{D}$ which is simply expressible by the Bergman's kernels $K_{\boldsymbol{D}_{n}}$ in the following way:

$$
\begin{aligned}
& K_{D}\left(z, z_{1}\right)=K_{D_{n}}\left(z, z_{1}\right) \text { if } z \text { and } z_{1} \text { belong to } D_{n}, \\
& K_{D}\left(z, z_{1}\right)=0 \text { if } z \text { and } z_{1} \text { belong to two different } D_{n} \text { 's. }
\end{aligned}
$$

There is no loss in generality if we suppose that $C$ is bounded (we can use conformal mappings to reduce every case to this one). We shall denote by $D_{0}$ the domain $D_{n}$ containing $\infty$.

Consider a decomposition of $C$ in two closed sets

$$
C=C^{(1)}+C^{(2)} \text {. }
$$

To every $C^{(i)}$ corresponds the complementary open set $D^{(i)}$ and we have

$$
D=D^{(1)} \cdot D^{(2)} \text {. }
$$

(13) The author introduced and investigated this kind of function in his thesis in Paris [2]. 
Every function $f(z)$ locally analytic in $D$ is decomposable into two functions $f(z)=f_{1}(z)+f_{2}(z)$ each of which is locally analytic in the corresponding domain $D^{(i)}$ (see N. Aronszajn [2]). Two such decompositions differ by a function with the singular set $C^{(1)} \cdot C^{(2)}$.

The classes $\mathfrak{A}_{D^{(i)}}$ are clearly linear closed subspaces of $\mathfrak{A}_{D}$ (when we restrict their functions to the set $D$ ).

The intersection of $\mathfrak{A}_{D^{(1)}}$ and $\mathfrak{A}_{D^{(2)}}$ is equal to the class $\mathfrak{A}_{D^{*}}$ where $D^{*}$ is the complementary set of $C^{*}=C^{(1)} \cdot C^{(2)}$.

The class $\mathfrak{A}_{D^{*}}$ is reduced to 0 when the intersection $C^{*}$ is a finite or an enumerable set.

The equality

$$
\mathfrak{A}_{D}=\mathfrak{A}_{D^{(1)}} \oplus \mathfrak{U}_{D^{(2)}}
$$

is not always true.

For simplicity's sake we suppose now that $C$ is not equal to 0 and is composed of a finite number of rectifiable arcs or closed curves, not reduced to isolated points, and that the same is true of $C^{(1)}$ and $C^{(2)}$.

It can then be proved that the necessary and sufficient condition in order that (6) be true is that $C^{*}=C^{(1)} \cdot C^{(2)} \neq 0$.

Further, if $C^{*}=0$, then $\mathfrak{H}_{D} \ominus\left[\mathfrak{H}_{D^{(1)}} \oplus \mathfrak{A}_{D^{(2)}}\right]$ is a one-dimensional space generated by the function $w^{\prime}(z)$ defined as follows: let $h(z) \equiv h_{C^{(1)}, C^{(2)}}$ be the locally harmonic function in $D$, taking the value 1 on $C^{(1)}$ and 0 on $C^{(2)}$. Denote then by $w(z)$ the function locally analytic in $D$ (but not necessarily single-valued in the multiply-connected components $D_{n}$ of $D$ ), whose real part is $h(z)$. The derivative $w^{\prime}(z)=h_{x}^{\prime}-i h_{y}^{\prime}$ is single-valued in $D$ and belongs to $\mathfrak{A}_{D} . h(z)$ is called the harmonic measure in $D$ corresponding to $C^{(1)}$.

From what we said, it results that we shall be able to calculate the kernel $K_{D}$ by the formulas of $\$ 12$ in the following cases:

(1) When $C^{*} \neq 0$ and $\mathfrak{A}_{D^{*}} \neq 0$ we can apply the general formula $(7), \S 12, \mathrm{I}$, translating operators into kernels, in particular $P, P_{0}, P_{1}$, and $P_{2}$ into $K_{D}$, $K_{D^{*}}, K_{D^{(1)}}$, and $K_{D^{(2)}}$. To calculate $K_{D}$ we have then to know $K_{D^{*}}, K_{D^{(1)}}$, and $K_{D^{(2)} \text {. }}$

(2) When $C^{*} \neq 0$ and $\mathfrak{A}_{D^{*}}=(0)$ we can apply formula (18) $\S 12, \mathrm{I}$.

This case contains interesting particular cases; for instance in the case of a simply-connected domain bounded by a polygonal line $C$. We can decompose $C$ into one side $C^{(1)}$ and a polygonal line $C^{(2)}$ having one side less than $C$. This gives an inductive process to calculate the kernel $K_{D}$ of the open set complementary to $C$. By this process we obtain, at the same time, the two Bergman's kernels for the interior and exterior of $C$. This is especially interesting as these kernels will give the conformal mapping functions of the domain on a circle.

Another case in point is one when to a slit $C^{(1)}$ in the plane we add a rectilinear segment $C^{(2)}$, having only one point in common with $C^{(1)}$. This 
case may be of interest for the variational methods, as they were used by Loewner [1], for instance, in the problem of coefficients of schlicht functions.

(3) When $C^{*}=0$, then $\mathfrak{A}_{D^{*}}=0$. We can apply formula (18), $\S 12, \mathrm{I}$, to compute the kernel of $\mathfrak{A}_{D^{(1)}} \oplus \mathfrak{A}_{D^{(2)}}$ if $K_{D^{(1)}}$ and $K_{D^{(2)}}$ are known. Then, if we know the function $w^{\prime}(z)$, we add the function $\left(1 /\left\|w^{\prime}\right\|{ }^{2}\right) w^{\prime}(z) \overline{w^{\prime}\left(z_{1}\right)}$ to the obtained kernel and arrive at the kernel $K_{D}$. The classes $\mathfrak{A}_{D^{(1)}}$ and $\mathfrak{A}_{D^{(2)}}$ have, in this case, a positive minimal angle for which a lower estimate can be obtained (using the constant $m$ from the inequality $m\left\|f_{1}\right\| \leqq\left\|f_{1}+f_{2}\right\|$ as in $\$ 12, \mathrm{I}$ ) by the use of methods developed by the author in [3].

If we consider the reduced classes $\mathfrak{A}_{D}^{\prime}$ of functions of $\mathfrak{A}_{D}$ with singlevalued integrals in every component domain $D_{n}$ of $D$, we shall obtain a r. k. $K_{D}^{\prime}$ expressible by a formula similar to (3) in terms of the reduced Bergman's kernels $K_{D_{n}}^{\prime}$. For the reduced classes we have always $\mathfrak{A}_{D}^{\prime}=\mathfrak{A}_{D}^{\prime}(1) \oplus \mathfrak{A}^{\prime} D^{(2)}$ and in the case $C^{*}=0$, we shall calculate $K_{D}^{\prime}$ directly by formula (18).

\section{BIBLIOGRAPHY}

N. Aronszajn

1. Sur les invariants des transformations dans le domaine de $n$ variables complexes, C. R. Acad. Sci. Paris vol. 197 (1933) p. 1579, and vol. 198 (1934) p. 143.

2. Sur les décompositions des fonctions analytiques uniformes et sur leurs applications, Acta Math. vol. 65 (1935) p. 1. (Thesis, Paris.)

3. Approximation des fonctions harmoniques et quelques problèmes de transformation conforme, Bull. Soc. Math. France vol. 67 (1939).

4. La théorie générale des noyaux reproduisants et ses applications, Première Partie, Proc. Cambridge Philos. Soc. vol. 39 (1944) p. 133.

5. Les noyaux pseudo-reproduisants. Noyaux pseudo-reproduisants et complétion des classes hilbertiennes. Completion fonctionnelle de certaines classes hilbertiennes.-Propriêtés de certaines classes hilbertiennes completées, C. R. Acad. Sci. Paris vol. 226 (1948) p. 456, p. 537 , p. 617 , p. 700.

6. Reproducing and pseudo-reproducing kernels and their application to the partial differential equations of physics, Studies in partial differential equations of physics, Harvard University, Graduate School of Engineering, Cambridge, Massachusetts, 1948.

S. BANACH

1. Theorie des opérations linéaires, Monografje Matematyczne, Warsaw, 1932.

\section{S. BERGMAN}

1. Ueber die Entwicklung der harmonischen Funktionen der Ebene und des Raumes nach Orthogonalfunktionen, Math. Ann. vol. 86 (1922) pp. 238-271. (Thesis, Berlin, 1921.)

2. Ueber die Bestimmung der Verzweigungspunkte eines hyperelliptischen Integrals aus seinen Periodizitätsmoduln mit Anwendungen auf die Theorie des Transformators, Math. Zeit. vol. 19 (1923) pp. 8-25.

3. Ueber die Bestimmung der elastischen Spannungen und Verschiebungen in einem konvexen Körper, Math. Ann. vol. 98 (1927) pp. 248-263.

4. Ueber unendliche Hermitesche Formen, die zu einem Bereich gehören, nebst Anwendungen auf Fragen der Abbildung durch Funktionen von zwei komplexen Veränderlichen, Berichte Berliner Mathematische Gesellschaft vol. 26 (1927) pp. 178-184, and Math. Zeit. vol. 29 (1929) pp. 640-677.

5. Ueber die Existenz von Repräsentantenbereichen, Math. Ann. vol. 102 (1929) pp. 430446. 
6. Ueber den Wertevorrat einer Funktion von zwei komplexen Veränderlichen, Math. Zeit. vol. 36 (1932) pp. 171-183.

7. Ueber die Kernfunktion eines Bereiches und ihr Verhalten am Rande, J. Reine Angew. Math. vol. 169 (1933) pp. 1-42, and vol. 172 (1934) pp. 89-128.

8. Sur quelques propriétés des transformations par un couple des fonctions de deux variables complexes, Atti della Accademia Nazionale dei Lincei, Rendiconti (6) vol. 19 (1934) pp. 474-478.

9. Zur Theorie von pseudokonformen Abbildungen, Rec. Math. (Mat. Sbornik) N.S. vol. 1 (1936) pp. 79-96.

10. Zur Theorie der Funktionen, die eine lineare partielle Differentialgleichung befriedigen, Rec. Math. (Mat. Sbornik) N.S. vol. 2 (1937) pp. 1169-1198.

11. Partial differential equations, advanced topics, Brown University, Providence, 1941.

12. A remark on the mapping of multiply-connected domains, Amer. J. Math. vol. 68 (1946) pp. 20-28.

13. Funktions satisfying certain partial differential equations of elliptic type and their representation, Duke Math. J. vol. 14 (1947) pp. 349-366.

14. Sur les fonctions orthogonales de plusieurs variables complexes avec les applications a la théorie des fonctions analytiques, Mémorial des Sciences Mathématiques, vol. 106, Paris, 1947.

S. Bergman and M. Schiffer

1. A representation of Green's and Neumann's functions in the theory of partial differential equations of second order, Duke Math. J. vol. 14 (1947) pp. 609-638.

2. On Green's and Neumann's functions in the theory of partial differential equations, Bull. Amer. Math. Soc. vol. 53 (1947) pp. 1141-1151.

3. Kernel functions in the theory of partial differential equations of elliptic type, Duke Math. J. vol. 15 (1948) pp. 535-566.

S. BOCHNER

1. Ueber orthogonale Systeme analytischer Funktionen, Math. Zeit. vol. 14 (1922) pp. 180207.

2. Vorlesungen ueber Fouriersche Integrale, Leipzig, 1932.

3. Hilbert distances and positive definite functions, Ann. of Math. vol. 42 (1941) pp. 647656.

J. DIXMIER

1. Sur une classe nouvelle de variêtés linéaires et d'opérateurs linéaires de l'espace d'Hilbert. Propriêtés gémetriques des domaines d'existence des opérateurs linéaires fermés de l'espace de Hilbert. Definition des opérateurs linéaires de l'espace d'Hilbert par leurs domaines d'existence et des valeurs, C. R. Acad. Sci. Paris vol. 223 (1946) p. 971, and vol. 224 (1947) p. 180 , p. 225.

K. FRIEDRICHS

1. On certain inequalities and characteristic value problems for analytic functions and for functions of two variables, Trans. Amer. Math. Soc. vol. 41 (1937) pp. 321-364.

B. FUCHS

1. Ueber einige Eigenschaften der pseudokonformen Abbildungen, Rec. Math. (Mat. Sbornik) N.S. vol. 1 (1936) pp. 569-574.

2. Ueber geodätische Mannigfaltigkeiten einer bei pseudokonformen Abbildungen invarianten Riemannschen Geometrie, Rec. Math. (Mat. Sbornik) N.S. vol. 2 (1937) pp. 567-594.

\section{P. Garabedian}

1. Schwarz' lemma and the Szegö kernel function, Trans. Amer. Math. Soc. vol. 67 (1949) pp. 1-35.

P. GARABEdian and M. Schiffer

1. Identities in the theory of conformal mapping, Trans. Amer. Math. Soc. vol. 65 (1948) pp. 187-238. 
I. GelFand and D. RaIKofF

1. Irreducible unitary representation of arbitrary locally bicompact groups, Rec. Math. (Mat. Sbornik) vol. 13 (1943) p. 316.

R. GoDEMENT

1. Sur les fonctions de type positif. Sur les propriêtés ergodiques des fonctions de type positif. Sur les partitions finies des fonctions de type positif. Sur certains opérateurs définis dans l'espace d'une fonction de type positif. Sur quelques propriêtés des fonctions de type positif définies sur un groupe quelconque, C. R. Acad. Sci. Paris vol. 221 (1945) p. 69, p. 134. and vol. 222 (1946) p. 36 , p. 213 , p. 529.

2. Les fonctions de type positif et la théorie des groupes, Trans. Amer. Math. Soc. vol. 63 (1948) pp. 1-84. (Thèse, Paris.)

J. KOBER

1. A theorem on Banach spaces, Compositio Math. vol. 7 (1939) pp. 135-140.

C. LOEWNER

1. Untersuchungen über schlichte konforme Abbildungen des Einheitskreises I, Math. Ann. vol. 89 (1923) pp. 103-121.

\section{J. MERCER}

1. Functions of positive and negative type and their connection with the theory of integral equations, Philos. Trans. Roy. Soc. London Ser. A vol. 209 (1909) pp. 415-446.

2. Sturm-Louville series of normal functions in the theory of integral equations, Philos. Trans. Roy. Soc. London Ser. A vol. 211 (1911) pp. 111-198.

E. H. Moore

1. On properly positive Hermitian matrices, Bull. Amer. Math. Soc. vol. 23 (1916) p. 59.

2. General analysis, Memoirs of the American Philosophical Society, Part I, 1935, Part II, 1939.

Z. NEHARI

1. The kernel function and canonical conformal maps, Duke Math. J. (1949).

2. On analytic functions possessing a positive real part, Duke Math. J. (1949).

J. v. Neumann and F. J. Murray

1. On rings of operators, Ann. of Math. vol. 37 (1936) pp. 116-229.

J. v. NeumanN and I. J. SCHOENBERG

1. Fourier integrals and metric geometry, Trans. Amer. Math. Soc. vol. 50 (1941) pp. 226-251.

M. SCHIFFER

1. The kernel function of an orthonormal system, Duke Math. J. vol. 13 (1945) pp. 529-540.

2. An application of orthonormal functions in the theory of conformal mappings, Amer. J. Math. vol. 70 (1948) pp. 147-156.

I. J. SCHÖNBERG

1. Metric spaces and positive definite functions, Trans. Amer. Math. Soc. vol. 44 (1938) pp. 522-536.

2. Positive definite functions on spheres, Duke Math. J. vol. 9 (1942) pp. 96-108.

M. H. STONe

1. Linear transformations in Hilbert space, Amer. Math. Soc. Colloquium Publications, vol. 15.

G. SzEGö

1. Ueber orthogonale Polynome, die zu einer gegebenen Kurve der komplexen Ebene gehoeren, Math. Zeit. vol. 9 (1921) pp. 218-270.

A. WEIL

1. L'intégration dans les groupes topologiques et ses applications, Actualités Scientifiques et Industrielles, vol. 869, Paris, 1940.

H. WELKE

1. Ueber die analytischen Abbildungen von Kreiskörpern und Hartogsschen Bereichen, Math. Ann. vol. 103 (1930) pp. 437-449. (Thesis, Münster, 1930.) 
K. ZaRANKIEWICZ

1. Ueber ein numerisches Verfahren zur konformen Abbildung zweifach zusammenhängender Gebiete, Zeitschrift für angewandte Mathematik und Mechanik vol. 14 (1934) pp. 97-104.

S. ZAREMBA

1. L'equation biharmonique et une classe remarquable de fonctions fondamentales harmoniques, Bulletin International de l'Académie des Sciences de Cracovie (1907) pp. 147-196.

2. Sur le calcul numérique des fonctions demandées dans le problème de Dirichlet et le problème hydrodynamique, Bulletin International de l'Académie des Sciences de Cracovie (1908) pp. 125-195.

Oklahoma Agricultural and Mechanical College, Stillwater, OKLA. 